\title{
Role of Neuronal Positioning in Jaw Motor Circuit Function in Zebrafish
}

\author{
A Dissertation \\ presented to \\ the Faculty of the Graduate School \\ at the University of Missouri-Columbia \\ In Partial Fulfillment \\ of the Requirements for the Degree \\ Doctor of Philosophy \\ by \\ Emilia Boakyewaa Asante \\ Dr. Anand Chandrasekhar, Dissertation Supervisor \\ May 2021
}


The undersigned, appointed by the dean of the Graduate School, have examined the dissertation entitled

\section{Role of Neuronal Positioning in Jaw Motor Circuit Function in Zebrafish}

presented by Emilia Boakyewaa Asante

a candidate for the degree of Doctor of Philosophy,

and hereby certify that, in their opinion, it is worthy of acceptance.

Dr. Anand Chandrasekhar

Dr. Elizabeth Bryda

Dr. Dawn Cornelison

Dr. David Schulz 


\section{ACKNOWLEDGEMENTS}

I would like to acknowledge the many people who have helped me during my doctoral journey.

I would like to thank the Chandrasekhar lab members, past and present, for their tremendous support over the years. I would like to thank my advisor Anand Chandrasekhar, for his guidance and support. He has consistently pushed me in my scientific work and greatly assisted me with my scientific writing. I am very grateful to you for providing me with opportunities to present and slowly overcome my fears. You truly challenged me to develop as a scientist, and I would be forever grateful to you.

I would like to thank Devynn Hummel for being my partner in crime and for being there to bounce off crazy ideas. I would also like to thank Suman Gurung for guiding me when I first joined the lab and keeping up with me even after leaving Mizzou. Your support and calming voice to my multiple texts have been invaluable. A very hardy thank you to all my undergraduates who have been helpful and fantastic lab members. I would like to thank Moe Baccam for taking care of the fish facility and being a great person to talk to and check-in with.

I am also grateful for not only belonging to the Chandrasekhar lab but to the Cornelison lab. The Cornelison lab members, past and present, have helped in countless ways. I would especially like to thank Robert Arpke, Lale Cecchini, Alex Diller, and Ronnie LaCombe for being there for 
me both in academics and in life. I enjoyed being your lab neighbor and, ultimately, lab member.

I would also like to thank my dissertation committee members, D Cornelison, Elizabeth Bryda, David Schulz, and Troy Zars, for providing me with excellent scientific suggestions as well as professional support. I would like to thank Troy Zars for the countless hours we spent before my comprehensive exam going over genetics.

Additionally, I would like to thank my friends and family. I would like to thank my friends Kazi Ramos and Niqua Hamilton, my west coast loves. You ladies have been there through it all and I cannot thank you enough. I would also like Dee Ainembabazi, Marla Bazan, and Rosi Molina my east coast loves. You ladies have been helpful to me, even from miles away. Thank you all for going on this ride with me! Your emotional support and check-ins have been tremendous. I would thank Candice King and Patricka Williams-Simon for the many adventures and for being a support system to me. From pep talks to celebrating milestones in life, you ladies have been awesome. We have become family and made Columbia home away from home. I cannot wait for the journey to continue in the coming years.

I would like to thank all the teachers and mentors that helped me get to where I am. I would like to thank the Lang Youth Medical Program, Joanne and Joe from the McNair program at Earlham College, and all my mentors from Bard High School Early College and Earlham college. And 
all the countless friends who became mentors who have come into my life and helped me immensely.

I would be remiss if I did not thank my family here in the US and Ghana. My journey wouldn't be possible without their help, support, and prayers. I would like to thank my mom and dad for being there for the latenight talks, daily prayers, and reassuring speeches. This degree is for you two! Thank you for showing up for me every time. I would also like to thank my brother Isaac, my sisters Sarah and Eunice, and my cousins Kwesi and Maame Kwatemaa. I would like to thank all my aunties and uncles for being the force beside me. Thanks to every single family member who called and checked in and was a listening ear. Finally, I would like to thank my grandmother Akosua Boakyewaa, a praying grandmother, for being the family force. I will be there this year for your 105th birthday Boakyewaa. Thank you for instilling in me a dream beyond our ancestors' wildest dreams. "Akwaaba! Meda wo ase"! 


\section{TABLE OF CONTENTS}

ACKNOWLEDGEMENTS.

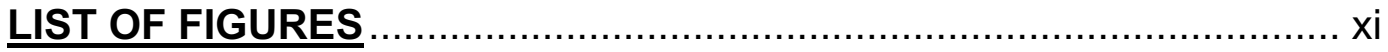

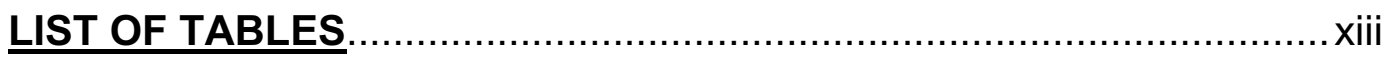

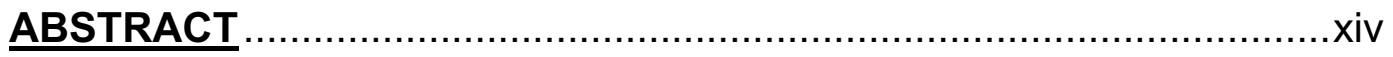

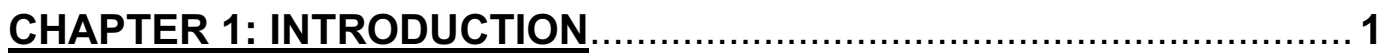

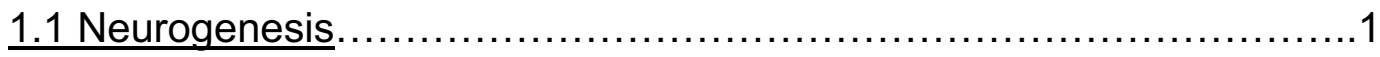

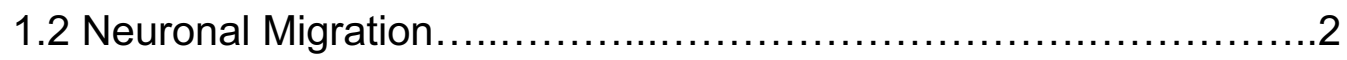

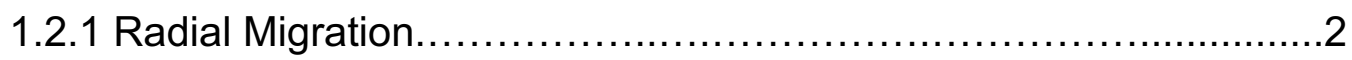

1.2.2 Tangential Migration................................................

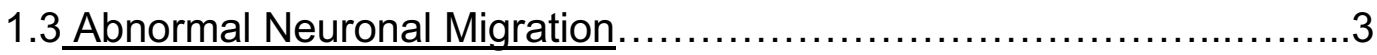

1.3.1 Lissencephaly .................................................... 4

1.3.2 Classical (Type I) Lissencephaly ................................. 4

1.3.3 Cobblestone (Type II) Lissencephaly..............................5

1.3.4 Periventricular Nodular Heterotopia..................................5

1.4 Insight Into Neuronal Migration Disorders Using Animal Models........6

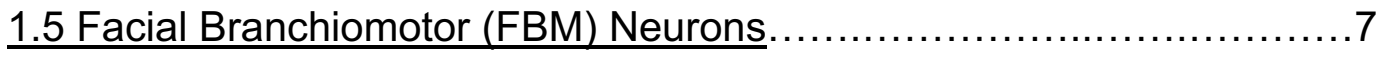

1.5.1 Functional Consequences of Defective FBM Neuron Migration.....8

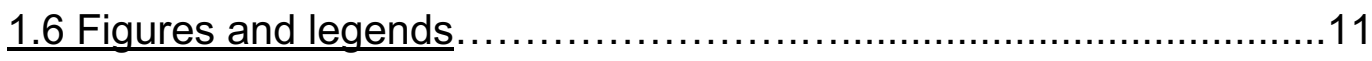

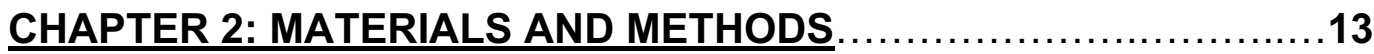

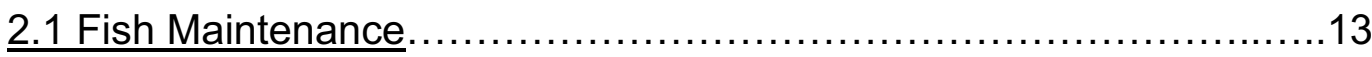

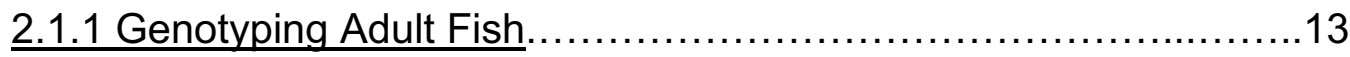

$\underline{2.2 \text { Zebrafish Lines } \ldots \ldots \ldots \ldots \ldots \ldots \ldots \ldots \ldots \ldots \ldots \ldots \ldots \ldots \ldots \ldots \ldots \ldots \ldots \ldots \ldots \ldots \ldots \ldots \ldots \ldots \ldots \ldots \ldots \ldots \ldots}$ 
2.2.1 $\operatorname{Tg}(i s / 1: g f p)$

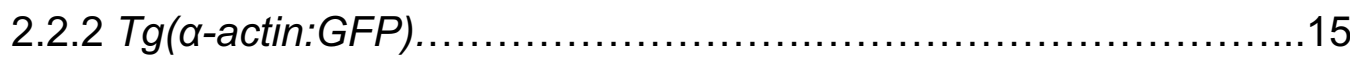

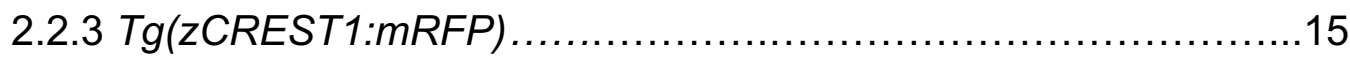

2.2.4 Tg(zCREST1:GCaMP6s)_....................................... 15

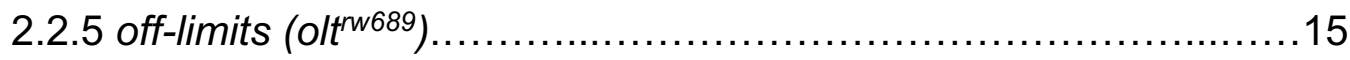

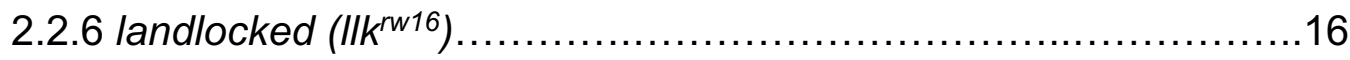

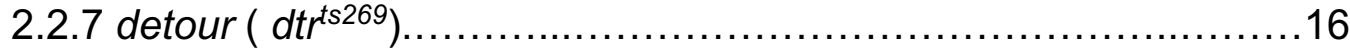

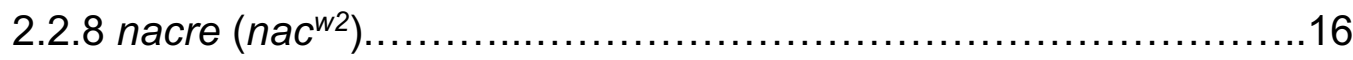

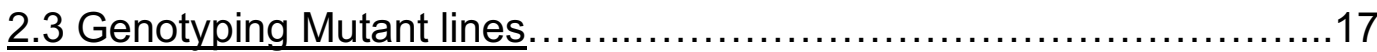

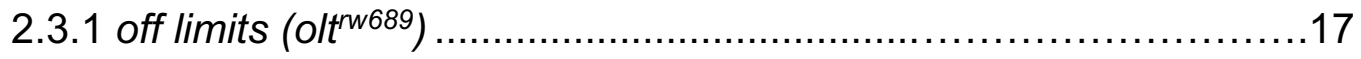

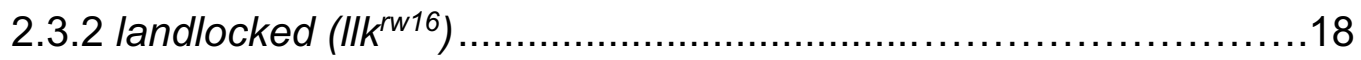

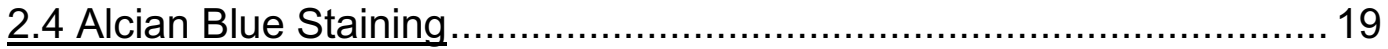

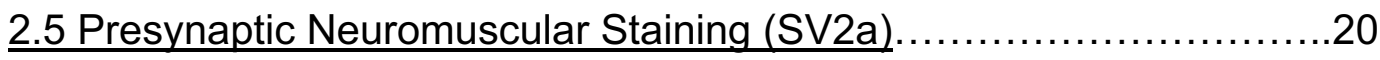

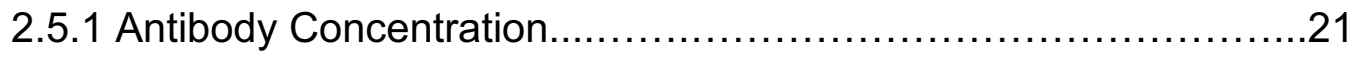

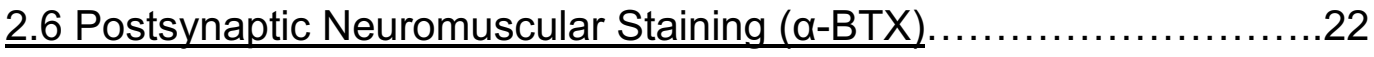

$\underline{2.7 \text { Calcium Imaging } \ldots \ldots \ldots \ldots \ldots \ldots \ldots \ldots \ldots \ldots \ldots \ldots \ldots \ldots \ldots \ldots \ldots \ldots \ldots \ldots \ldots \ldots \ldots \ldots \ldots \ldots \ldots \ldots}$

$\underline{2.8 \text { Confocal Imaging } \ldots \ldots \ldots \ldots \ldots \ldots \ldots \ldots \ldots \ldots \ldots \ldots \ldots \ldots \ldots \ldots \ldots \ldots \ldots \ldots \ldots \ldots \ldots \ldots \ldots \ldots \ldots \ldots}$

$\underline{2.9}$ Measurement of Axon morphologies Using LAS X Software............23

2.10 NMJ Measurements on Jaw Muscles Using LAS X Software..........24

$\underline{2.11 \text { Food Intake Assay } \ldots \ldots \ldots \ldots \ldots \ldots \ldots \ldots \ldots \ldots \ldots \ldots \ldots \ldots \ldots \ldots \ldots \ldots \ldots \ldots \ldots \ldots \ldots \ldots \ldots}$

2.11.1 Preparation of Fluorescent Larval Food.........................29

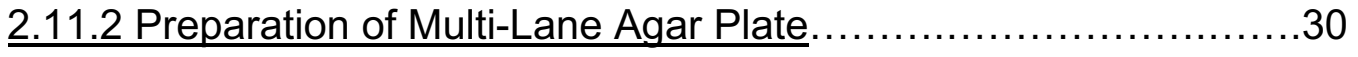


2.11.3 Preparation of $3 \%$ Methyl Cellulose With $0.016 \%$ Tricaine .......30

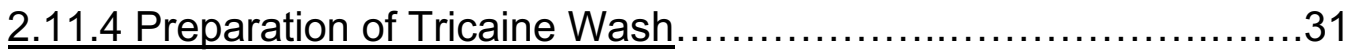

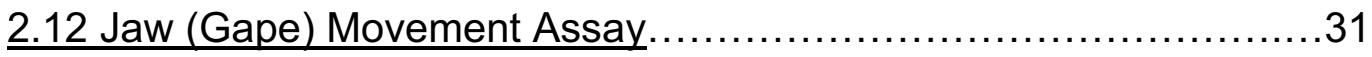

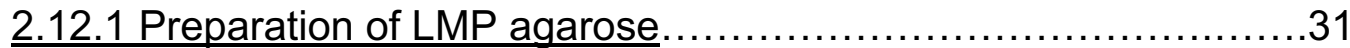

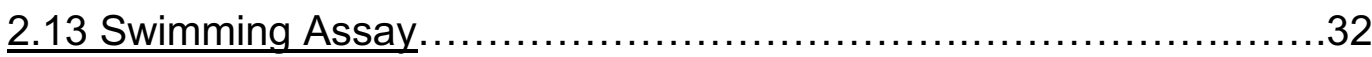

CHAPTER 3: DISSECTING BRANCHIOMOTOR NEURON CIRCUITS IN ZEBRAFISH TOWARD HIGH-THROUGHPUT AUTOMATED ANALYSIS

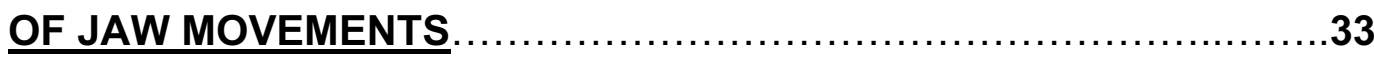

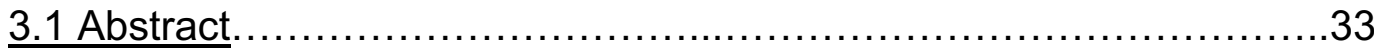

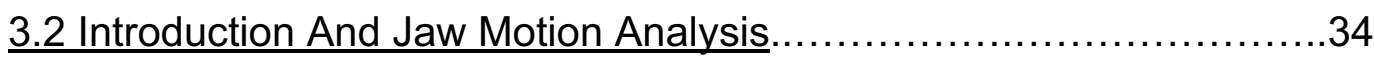

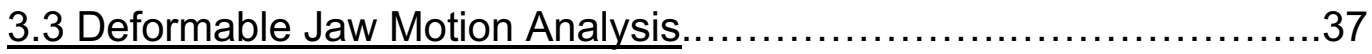

3.3.1 Mutiple Motion Models of Jaw Movements............................37

3.3.2 Proposed Robust Fusion of Motion Fields.............................38

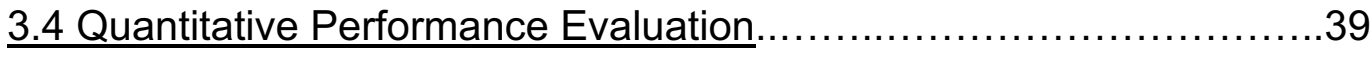

3.5 Experimental Results And Disscussion................................40

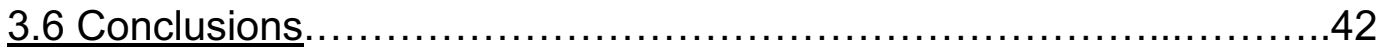

3.7 Acknowledgments......................................................

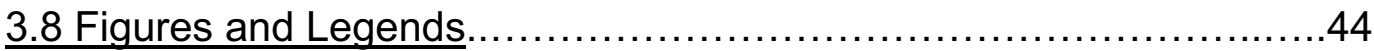

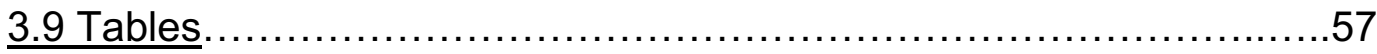

CHAPTER 4: DEFECTS IN NEURONAL POSITIONING IMPACT MOTOR CIRCUIT FUNCTION IN ZEBRAFISH $\ldots \ldots \ldots \ldots \ldots \ldots \ldots \ldots \ldots . \ldots 58$

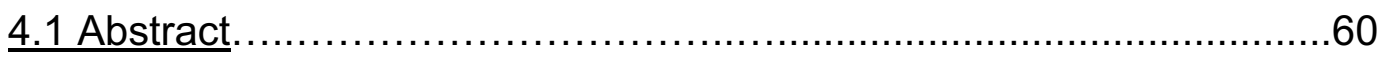

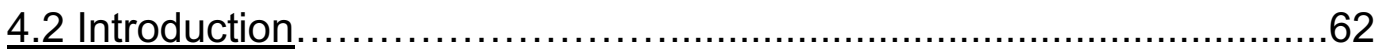


4.3.1 Ontogeny of Lower Jaw Movement In Wildtype Zebrafish

Larvae. .66

4.3.2 Changes in Branching And Pre-Synaptic Structures of Branchiomotor Axons May Drive Increases in Jaw Motor Output

4.3.3 Jaw Movement is Defective in the off limits (olt) Neuronal Migration

Mutant. 69

4.3.4 Defective Jaw Movements in olt Mutants Are Associated With

Reduced Food Intake.

4.3.5 FBM Axon Outgrowth to Jaw Muscles Occurs Normally in olt

Mutants.

4.3.6 FBM Axons Extending on The Ih Muscle Are Frequently

Defasciculated in olt Mutants .73

4.3.7 NMJs on Jaw Muscles Appear to be Unaffected in olt

Mutants. .74

4.3.8 FBM Neurons Are Less Active in olt Mutants. .75

$\underline{4.4 \text { Discussion. }}$ .76

4.4.1 Developmental Changes in Jaw Movement Behavior May be Pre-

Synaptically Driven. .77

4.4.2 Defective FBM Neuron Position in olt Mutants is Associated With

a Significant Functional Deficit in The Motor Circuit. .79

4.4.3 Are Branchiomotor Axon And Activity Defects in olt Mutants a 


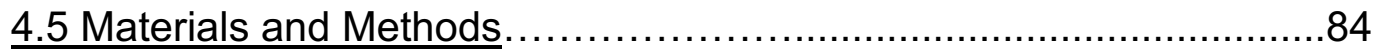

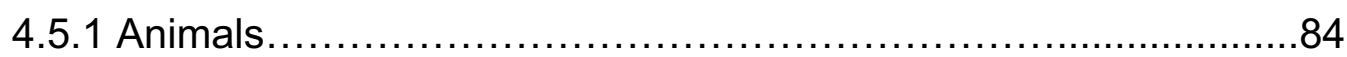

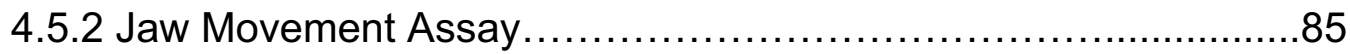

4.5.3 Food Intake (Feeding) Assay ........................................ 86

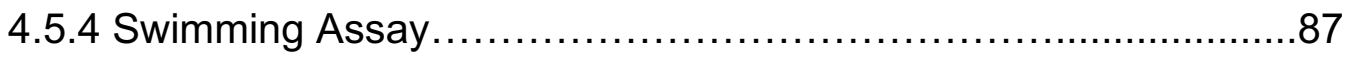

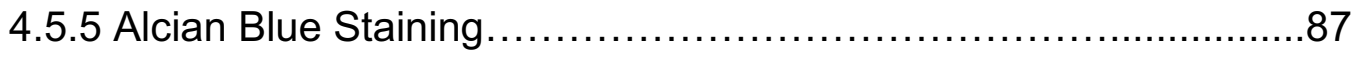

4.5.6 Immunostaining And Analysis of Neuromuscular Junctions..........88

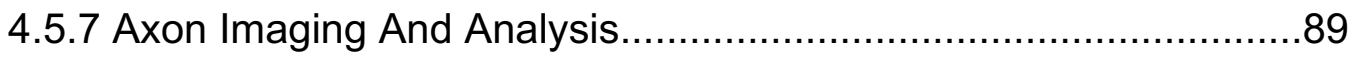

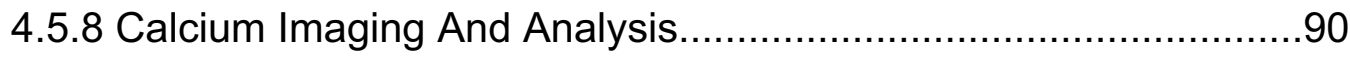

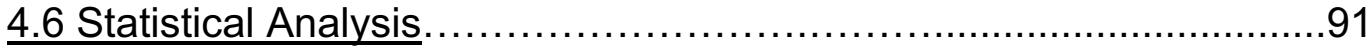

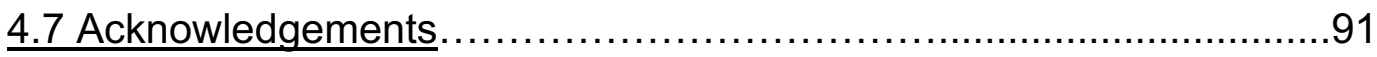

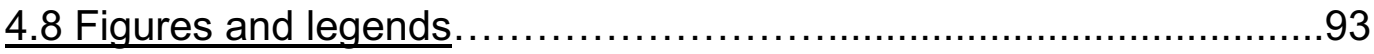

4.9 Supplementary Figures and Legends .........................................110

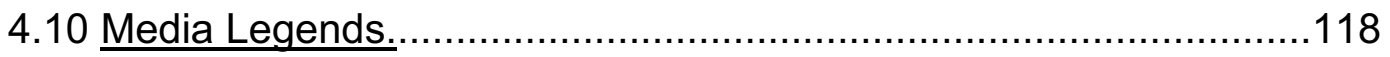

CHAPTER 5: FUTURE DIRECTIONS AND CONCLUSIONS $\ldots \ldots \ldots \ldots . . . . .120$

5.1 The functional consequences of defective FBM neuron migration in zebrafish Mutant Landlocked (Ilk) ...............................................121

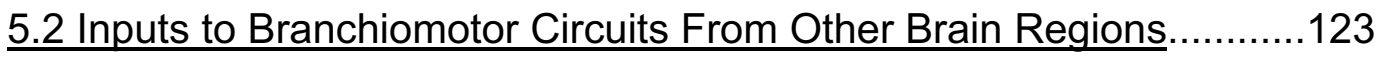

5.2.1 Input From The Visual System to the Branchiomotor Circuits.......124

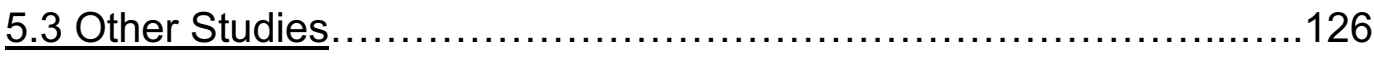

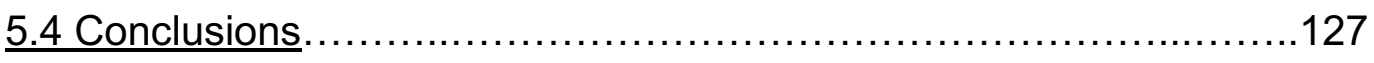


5.4.1 Tangential Migration Defect in the Developing Brain.

5.4.2 Pursuing the Link Between Neuronal Position and

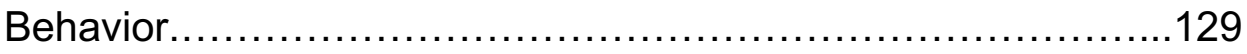

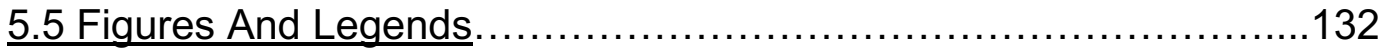

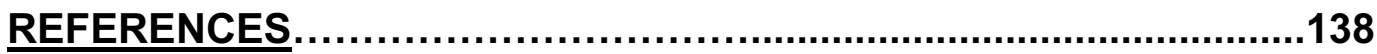

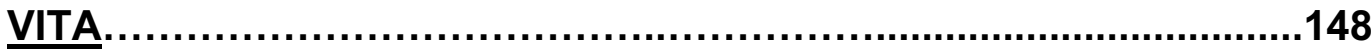




\section{LIST OF FIGURES}

Figure 1.1 Schematic of neuronal migration disorders (NMDs). 11

Figure 3.1 Manual workflow for zebrafish jaw movement analysis 44

Figure 3.2 Branchiomotor neurons in $3 \mathrm{dpf}$ zebrafish larvae .46

Figure 3.3 High-throughput image analytics pipeline .48

Figure 3.4 Mispositioned BM neurons in larval brain........................50

Figure 3.5 Plot of gape count vs video number .........................52

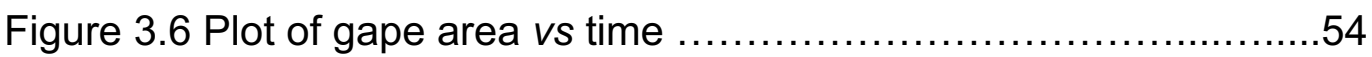

Figure 4.1 Ontogeny of lower jaw movement in wildtype zebrafish larvae.

Figure 4.2 Developmental changes in branchiomotor axon branching and synaptic structures at the jaw neuromusclar junction.....................95

Figure 4.3 olt mutants have reduced jaw movement .....................98

Figure 4.4 Defective jaw movements greatly reduce food intake in olt mutants 100

Figure 4.5 Axon guidance and outgrowth are unaffected in olt mutant larvae 103

Figure 4.6 Neuromuscular junctions on jaw muscles in olt mutants are unaffected 106

Figure 4.7 FBM neurons are less active in olt mutants. 108

Figure 4.1.1: System for imaging jaw movement and processing steps in motion analysis software 110 
Figure 4.1.2: Morphometric analysis of the heads of 5 and $7 \mathrm{dpf}$ larvae.......

Figure 4.1.3 Measurement of volumes of presynaptic and postsynaptic structures at the NMJs. 114

Figure 4.1.4: Measurement of motor axon lengths and branch

numbers

Figure 5.1: Ilk mutants have reduced food intake....

Figure 5.2: Jaw movement is increased in neuronal migration mutant Ilk. 134

Figure 5.3: Inputs to branchiomotor circuits from other brain regions....136 


\section{LIST OF TABLES}

Table 3.1 Quantitative evaluation at the frame correspondence level for

three different age groups of zebrafish larvae ................................... 158 


\begin{abstract}
Development of the vertebrate nervous system involves substantial cell migration, where immature neurons move to specific locations to generate functional circuits. Precise neuronal migration and positioning are essential for proper brain architecture and function.

Abnormal neuronal migration can contribute to neurological disorders such as lissencephaly, autism and schizophrenia. However, the consequences of abnormal neuronal migration for circuit organization and functional output are poorly understood. To provide some insight, I used the facial branchiomotor (FBM) neurons in zebrafish as a model system to analyze the effects of aberrant neuronal migration on circuit function.

The FBM neurons are a subset of the branchiomotor neurons, which are generated in the vertebrate hindbrain and innervate facial and jaw muscles. During development in zebrafish and mice, FBM neurons migrate caudally from rhombomere $4(r 4)$ to $r 6$ to form the facial motor nucleus and innervate jaw and gill muscles (in fish). In order to examine the consequences of aberrant neuronal migration, one must first characterize the normal functional output of the FBM circuit that drives jaw movements. In collaboration with colleagues in the MU Department of Computer Science, we developed an automated image analysis system to extract motion features from video recordings of jaw movement, enabling rapid and accurate high-throughput analysis. We used this software to examine the emergence of jaw movement in zebrafish larvae between 3-9
\end{abstract}


days post fertilization (dpf). While gape, the displacement of the lower jaw to form the mouth opening, was minimal at $3 \mathrm{dpf}$, gape frequency increased sharply by $5 \mathrm{dpf}$, and stabilized by $7 \mathrm{dpf}$. A detailed analysis of branchiomotor axons and neuromuscular junctions (NMJs) on jaw muscles suggest that this "maturation" of branchiomotor circuit output may be driven by changes in presynaptic structures at the jaw NMJs.

To evaluate the consequences of defective neuronal migration on circuit output, I examined whether jaw movement was affected in the zebrafish off-limits (olt) mutant in which FBM neurons fail to migrate out of r4. In olt mutants, the increase in gape frequency occurred normally between 3-5 dpf. However, the average gape frequency was $~ 50 \%$ lower than wildtype siblings from 5-9 dpf while gape amplitude was unaffected. Given the jaw movement defect in olt mutants, I evaluated food intake, an independent measure of jaw movement and another functional output of the branchiomotor circuit. Olt mutants ate poorly compared to their wildtype siblings, consistent with their reduced jaw movement.

I then tested several potential mechanisms that could generate the functional deficits in olt mutants. While $f z d 3 a$, the gene inactivated in olt mutants, is ubiquitously expressed in neural and non-neural tissues, jaw cartilage and muscle developed normally in olt mutants, and muscle function also appeared to be unaffected. Although FBM neurons were mispositioned in olt mutants, axon pathfinding to jaw muscles were unaffected. Moreover, neuromuscular junctions established by FBM 
neurons on jaw muscles were similar between wildtype siblings and olt mutants. Interestingly, FBM axons innervating the interhyoideus jaw muscle were frequently defasciculated in olt mutants. Furthermore, GCaMP imaging revealed that mutant FBM neurons were less active than their wildtype counterparts. These data suggest that aberrant positioning of FBM neurons in olt mutants results in subtle defects in fasciculation and neuronal activity, potentially generating defective functional outputs.

In the future, we will examine modulatory inputs from other brain regions to the branchiomotor neurons and examine their roles in impacting circuit output in olt mutants. 


\section{CHAPTER 1: INTRODUCTION}

During development, precise migration of neurons are essential to the proper function of the brain. When neuronal migration is defective, it can have severe consequences and result in aberrant behavior and disease. Given the critical role of migration, it is important to understand the functional outputs of abnormal neuronal migration in the vertebrate brain.

\subsection{Neurogenesis}

Neural progenitor cells of the central nervous system (CNS) are composed of neuroepithelial cells (NECs) and radial glial (RG) cells (Rakic, 1972). NECs divide symmetrically to self-renew and increase the pool of founder progenitors. NECs transform into RG cells and acquire the potential to generate neurons and glia (Noctor et al., 2002; Morest and Silver, 2003). RG cells are self-renewing cells that generate neurons via symmetrical and asymmetrical divisions in the ventricular zone (VZ). During asymmetrical divisions, RG cells produce a self-renewed RG cell and a neuronal daughter cell (Noctor et al., 2001; Evsyukova et al., 2013). RG cells divide in the VZ and produce intermediate progenitor (IP) cells. IP cells divide in the subventricular zone to produce pairs of daughter neurons (Noctor et al., 2004, 2008). These patterns of amplifying divisions in the cerebral cortex increase the number of cells during the period of neurogenesis. Migrating neurons develop the cortex into six-layered 
structures (I-VI), and newborn neurons adopt two modes of migration in the CNS, radial and tangential migration (Nadarajah and Parnavelas, 2002; Kanatani et al., 2005).

\subsection{Neuronal Migration}

A fundamental rule of the developing nervous system is that cells are generated in sites different from where they later reside. Neurons do not move in aimlessly in the CNS; they adopt two main strategies, radial and tangential migration (Hatten, 1999; Marín and Rubenstein, 2003).

\subsubsection{Radial Migration}

In radial migration, neurons use radial glial fibers as a substrate, extend a leading process, and move the nucleus through the elongated process (Noctor et al., 2001; Nadarajah, 2003). Migration along radial glial fibers is central to the architecture of the cortex (Nadarajah, 2003). Neurons move from the VZ to the pial surface, preplate layer, marginal zone, and the cortical plate layer when the cerebral wall is thin. These layers are the earlier cortical framework, and as such, they are generated in an inside-out manner, with the early-born neurons in the deepest layers and the later-born cells forming the superficial layers (Nadarajah et al., 2001; Nadarajah and Parnavelas, 2002). Later born neurons have short bursts of movement with pauses as they migrate along glial fibers. 
Migration along radial glial fibers is central to the architecture of the cortex (Nadarajah, 2003).

\subsubsection{Tangential Migration}

During tangential migration, neurons follow a trajectory that is parallel to the ventricular surface and orthogonal to the orientation of the radial glial fiber (O’Rourke et al., 1992, 1995; Marín and Rubenstein, 2001). While radially migrating neurons use radial glial fibers as a substrate, tangentially migrating neurons do not require such support. Tangentially migrating neurons can move in clusters and interact with other cell types, as seen for olfactory bulb interneurons. Conversely, they can move individually, as seen for Cajal-Retzius cells and cortical interneurons (Hatten, 1999; Marín and Rubenstein, 2001; Marín et al., 2010). Tangentially migrating neurons that interact with each other respond to cues present in the extracellular matrix or on the surface of the other cells to undergo directional migration (Marín et al., 2010).

\subsection{Abnormal Neuronal Migration}

Deficiencies in the process of neuronal migration can lead to several diseases (Fig. 1.1). Neuronal migration disorders (NMDs) are birth defects caused by the abnormal migration of neurons in the developing neocortex (Guarnieri et al., 2018). NMDs vary in the degree of cortical malformations and structural abnormalities. Disruption of neuronal 
migration can result in severe cognitive deficits, intractable seizures, and impair motor movements. Studies into the causes of NMDs have identified genes associated with neuronal migration defects (Liu, 2011; Jiang and Nardelli, 2016).

\subsubsection{Lissencephaly}

In a normal brain, many neurons migrate radially from the VZ into the neocortex. In a lissencephalic brain, these neurons are either halted or over-migrate onto the surface of the cortex (Gleeson and Walsh, 2000), presenting a 'smooth brain' in which there is a loss of the gyri and sulci of the brain (Fig. 1.1 C-F). Several types of lissencephaly have been recognized, with classical lissencephaly (type 1) and cobblestone lissencephaly (type II) being the most common ones (Pang et al., 2008; Guarnieri et al., 2018).

\subsubsection{Classical (Type I) Lissencephaly}

Type 1 lissencephaly consists of failure of the neurons to migrate, resulting in four layers in the cerebral cortex (Moffat et al., 2015) (Fig. 1.1 C-D). Type 1 lissencephaly results in poor motor function, difficulties feeding, seizures, and varying levels of intellectual disabilities (Dabyns and Truwit, 1995; Fox and Walsh, 1999). Several genes have been associated with type 1 lissencephaly, such as Lissencephaly (LIS1) and 
Doublecortin (DCX). Mouse disease models for these genes exhibit similar phenotypes to those seen in patients (Gleeson, 2001; Pang et al., 2008; Reiner, 2013).

\subsubsection{Cobblestone (Type II) Lissencephaly}

In type II lissencephaly, neurons over-migrate through the pial surface and present with no distinguishable layer and a cobblestone appearance (Dabyns and Truwit, 1995) (Fig. 1.1 E-F). Type II lissencephaly is associated with various eye abnormalities and congenital muscular dystrophies. Several genetic mutations are linked to Type II lissencephaly, with the most severe form associated with mutations in the FKTN (Fukuyama syndrome or Walker-Warburg Syndrome) (Fox and Walsh, 1999; Moffat et al., 2015).

\subsubsection{Periventricular Nodular Heterotopia}

Periventricular nodular heterotopia $(\mathrm{PH})$ is a condition in which neurons fail to migrate from the ventricular zone, which leads to the formation of nodules along the lateral ventricles (Fig. 1.1 G-H). PH can be caused by mutations in several genes such as FilaminA (FLNA) and adenosine diphosphate-ribosylation factor guanine exchange factor 2 (ARFGEF2) (Guerrini and Dobyns, 2014). PH associated with the FLNA gene is inherited in an X-linked dominant pattern, and affected individuals 
are usually only discovered after presenting with epilepsy and magnetic resonance imaging (MRI) studies confirm the condition. Some patients have a mild intellectual disability such as dyslexia (Fox and Walsh, 1999; Gleeson, 2001). A much rarer autosomal recessive form of $\mathrm{PH}$ due to ARFGEF2 mutations leads to microcephaly, epilepsy, and severe developmental delays (Lu and Sheen, 2005; Guerrini and Dobyns, 2014).

\subsection{Insight Into Neuronal Migration Disorders}

\section{Using Animal Models}

Advances in engineering animal models of cortical malformations have revealed the diversity and complexity of NMDs. Some NMD patients present with obvious neurological impairment, whereas others show unexpected deficits that are detectable only by screening and imaging. A comprehensive animal model analysis is needed to accurately define the functional behaviors in these disorders and develop potential therapeutics.

Mouse models have recapitulated some aspects of human NMDs, and several models of lissencephaly have been generated based on the mutations associated with LIS1 (Reiner et al., 1993), DCX (Des Portes et al., 1998), and CEP85L (Tsai et al., 2020). Additionally, mutagenesis has identified mouse genes such as TUBA1A that generated phenotypes resembling previously uncharacterized human NMDs (Keays et al., 2007). Progress in generating transgenic models of NMDs has allowed for the identification of cellular and molecular mechanisms and provided 
translationally relevant insights into the pathogenesis of NMDs. Loss of function of Lis1 in mouse mutants resulted in neuronal migration defect and intractable seizures (Fleck et al., 2000; Greenwood et al., 2009). Furthermore, in vivo studies inhibiting Lis1 protein degradation were sufficient to partially rescue neuronal migration deficits and ameliorate behavioral symptoms in Lis1 mutant mice (Yamada et al., 2009, 2010). Animal models have broadened our knowledge of disease mechanisms and behavioral deficits associated with defective neuronal positioning.

While the challenges of moving animal studies into human patients cannot be overstated (Buchsbaum and Cappello, 2019), transgenic animal models of NMDs provide critical approaches to explore the relationship between neuronal migration defects and behavioral abnormalities. The consequences of abnormal neuronal migration on circuit organization and behavioral function are an ongoing and ever-evolving area of neuroscience. To investigate neuronal migration and functional output, we study the facial branchiomotor (FBM) neurons in zebrafish.

\subsection{Facial Branchiomotor (FBM) Neurons}

The facial branchiomotor (FBM) neurons, a subset of branchiomotor neurons, is an excellent model to study neuronal migration due to their evolutionarily conserved and stereotyped migration pattern (Chandrasekhar, 2004; Song, 2007). Branchiomotor neurons are generated in specific segments of the embryonic hindbrain called 
rhombomeres, and innervate muscles forming in the pharyngeal arches. In all model vertebrates studied except chicken, FBM neurons migrate tangentially from rhombomere (r)4 into r6/7. FBM axons exit the hindbrain from $r 4$ into the second pharyngeal arch and innervate muscles of facial expression, jaw movements, the middle ear, and upper neck (Graham and Smith, 2001; Chandrasekhar, 2004). Recent studies utilizing mouse and zebrafish models have demonstrated the importance of precise positioning to establish appropriate structural organization and function.

\subsubsection{Functional Consequences of Defective FBM}

\section{Neuron Migration}

In the Van gogh-like 2 (Vang/2) mouse mutant, looptail (Lp), FBM neurons fail to migrate from $r 4$ into $r 6$ due to loss of function of the Wnt/Planar Cell Polarity (PCP) protein Vangl2 (Glasco et al., 2012). During development, the embryonic parafacial oscillator (e-pF) respiratory neurons arise adjacent to FBM neurons; hence FBM neuron migration defects might affect e-pF neuron positioning and function. Therefore, the functional output of the respiratory neuronal network was examined in $L p$ mutants (Thoby-Brisson et al., 2012). In $L p$ mutants, e-pF neurons were substantially mispositioned, yet the e-pF respiratory oscillators formed functional connections with each other across the midline and with the preBotC oscillators in the caudal hindbrain (Thoby-Brisson et al., 2012). These results indicate that neuronal networks are important for 
physiological function and survival and are robust and resistant to changes in neurons' positions.

In zebrafish, landlocked (Ilk) and prickle1b (pk1b) mutants (Wada et al., 2005; McArthur and Fetcho, 2017), FBM neurons fail to migrate out of r4 but still appear to innervate jaw muscles. Although the mutation in these two lines caused motor neuron cell bodies and dendritic arbors to misposition in the mutants, the organization of neurons within the motor nucleus according to age and target muscle was not affected (McArthur and Fetcho, 2017). The structural and functional organization robustness to the aberrant segmental position is poorly understood. Further investigation into the functional output of the FBM neurons is crucial.

To evaluate the branchiomotor network, we developed two assays to measure the functional readout of the FBM neuron circuit. $\underline{A \text { proprietary }}$ software facilitated analysis of jaw movements in zebrafish larvae in

Chapter 3 . And a qualitative food intake assay in 7 days post-fertilization (dpf) zebrafish larvae (Allen et al., 2017). Larvae were fed fluorescent microspheres coated with fish food 3 hours, and the fluorescent contents in their gut were quantified to assign a feeding score. Chemical and laser ablation of branchiomotor neurons in $7 \mathrm{dpf}$ zebrafish predicably reduced food intake without affecting swimming ability. This behavior demonstrated that our feeding assay was sensitive and robust. We then examined food intake in detour (gli1-/-) mutant fish, where cranial motor neurons are not induced but spinal motor neurons develop normally. Food intake by $7 \mathrm{dpf}$ 
detour mutant larvae was almost completely abolished; many larvae had no fluorescence in the gut (Allen et al., 2017). These experiments demonstrated that the complete ablation of FBM neurons either chemically or genetically significantly impacts the circuit's functional output. However, it is unclear the functional output in an aberrant neuronal migration mutant. Therefore, in Chapter 4, I examined the consequences of abnormal neuronal migration on the neural circuit driving motor behaviors essential for survival. 


\subsection{FIGURES AND LEGENDS}

Figure 1.1 Schematic of neuronal migration disorders (NMDs).

A

Normal
brain

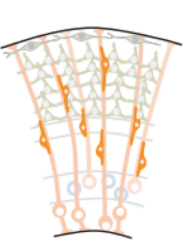

B

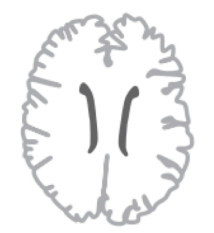

C Lissencephaly type 1

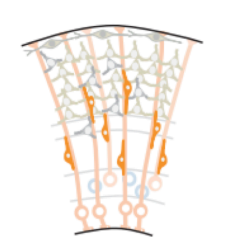

D

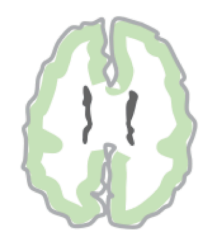

E Lissencephaly type II

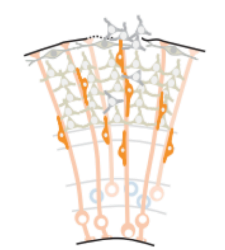

$\mathbf{F}$

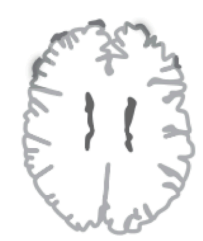

G

Periventricular heterotopia

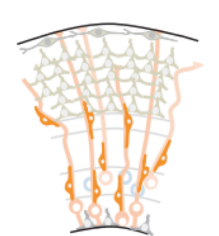

H

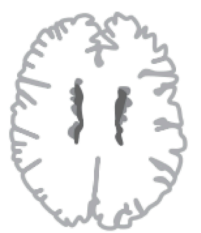


Figure 1.1 Schematic of neuronal migration disorders (NMDs).

Neuronal migration disorders (NMDs). (A,C,E,G) schematics of the cellular composition of different NMDs in the human cortex, indicating brain layers. Aberrant neurons are shown in dark gray. $(B, D, F, H)$ Schematic of MRI images of NMDs in the human brain, defective neuronal position is shown as grey shading for each disorder. Lissencephaly type I has a four-layered cortex (green shading). Schematics are adapted from (Buchsbaum and Cappello, 2019). 


\section{CHAPTER 2: MATERIAL AND METHODS}

\subsection{Fish Maintenance}

Zebrafish (Danio rerio) were maintained on a 14 hour light/10 hour dark cycle as described in (Westerfield, 1995). Embryos were obtained by placing 2-3 pairs of adult fish in a mating cage. Embryos were grown at $28.5^{\circ} \mathrm{C}$ in a 14 -hour light, 10 -hour dark cycles (LD 14:10) incubator in embryo medium (E3). Embryos were precisely staged at the 4-8 cell stage, and again during somitogenesis (16-20 hpf) by counting somites. Subsequently, embryos and larvae were staged by hours or days post fertilization (hpf or dpf) (Kimmel et al., 1995). Embryos were treated with phenylthiourea (PTU, Sigma) between 18-24 hpf to block pigmentation. Embryos were placed in LD 14:10 incubator until ready for use in experiments. Larvae were fixed in $4 \%$ paraformaldehyde at required timepoints for analysis.

\subsubsection{Genotyping Adult Fish}

Adult zebrafish were anesthetized using tricaine (MS222, Western Chemical Co.) made by adding $10 \mathrm{ml}$ of stock tricaine $(0.4 \%)$ to $240 \mathrm{ml}$ of fish water and brought to $\mathrm{pH} 7.0$ using sodium bicarbonate. After the fish were anesthetized briefly for $\sim 1-2$ minutes, their caudal fin was clipped between the tip of fin to the edge where the scales end. The fish were 
placed in individual holding tanks to regenerate the fin for two weeks before pooling fish of the same genotypes. Fin clip tissue was placed in $0.5 \mathrm{ml}$ of lysis buffer and digested overnight with rocking in a $55^{\circ} \mathrm{C}$ incubator. The next day, samples were centrifuged (Eppendorf Tabletop) for 20 minutes at room temperature. The supernatant containing DNA was transferred to a labeled $1.5 \mathrm{ml}$ centrifuge tubes containing $0.5 \mathrm{ml} 100 \%$ ethanol. The samples were rocked for 1-3 hours at a moderate speed at room temperature until white/yellow strands were seen. The DNA was pelleted by centrifuging at $4^{\circ} \mathrm{C}$ for 20 minutes. The pellet was washed in cold $70 \%$ ethanol and centrifuged at $4^{\circ} \mathrm{C}$ for 20 minutes. The ethanol was removed, and the pellet was air-dried for 10-15 minutes at room temperature. The DNA was resuspended in $20 \mu \mathrm{l}$ sterile $\mathrm{H}_{2} \mathrm{O}$ and stored at $4^{\circ} \mathrm{C} .2 \mu \mathrm{l}$ of the resuspended DNA was used in a $25 \mu \mathrm{l}$ PCR reaction.

\subsection{Zebrafish Lines}

\subsubsection{Tg(is/1:gfp)}

The $T g(i s / 1: g f p)$ transgenic line (Higashijima et al., 2000) expresses green fluorescent protein (GFP) in the cranial motor neurons ( $\mathrm{nV}, \mathrm{nVII}$, $n X)$, except the nIX motor neurons. This line enables visualization of the cell bodies, the primary axon fascicles and the peripheral branches. This GFP transgenic line was used to examine the developmental emergence of jaw movement data in wildtype larvae. This line was provided by Dr. Hitoshi Okamoto (RIKEN, Japan). 


\subsubsection{Tg(a-actin:GFP)}

The $\operatorname{Tg}(\alpha$-actin:GFP) transgenic line (Higashijima et al., 1997) expresses green fluorescent protein (GFP) in muscle cells. This line was provided by Dr. Hitoshi Okamoto (RIKEN, Japan).

\subsubsection{Tg(zCREST1:mRFP)}

The $z C R E S T 1: m R F P$ transgenic line was generated using the zCREST1 enhancer, the $h s p 70 /$ promoter and membrane-localized RFP to drive expression in branchiomotor neurons (Mapp et al., 2010). The zCREST1 is an enhancer element within the islet1 promoter and drives expression in the cranial motor neurons (Uemura et al., 2005). This line was provided by Dr. Victoria Prince (University of Chicago).

\subsubsection{Tg(zCREST1:GCaMP6s)}

Tg(zCREST1:GCaMP6s) was generated by Devynn Hummel in the Chandrasekhar lab by Tol2 transgenesis. The Tol2 destination vector was generated by Gateway Cloning of p5E-zCREST1, pENTR-GCaMP6s (provided by Dr. Lila Solnica-Krezel, Washington University), and p3EpolyA constructs. This line expresses GCaMP6s in branchiomotor neurons at low levels until $48 \mathrm{hpf}$, at a much higher level between 4-7 dpf (days post fertilization). 


\subsection{5 off-limits (olt $\left.{ }^{r w 689}\right)$}

The off-limits (olt) locus encodes the frizzled3a $(f z 3 a)$ gene. The olt ${ }^{r w 689}$ allele carries a mis-sense mutation which disrupts proper folding of the Frizzled protein (Wada et al., 2006). In the absence of olt expression in the neuroepithelium, zygotic as well as maternal-zygotic embryos display facial motor (FBM) neuron migration defects. This line was provided by Dr. Hitoshi Okamoto (RIKEN, Japan).

\subsection{6 landlocked (II/ $\left.{ }^{r w 16}\right)$}

The landlocked (Ilk) locus encodes the scribble1 (scrb1) gene. The $\| k^{r w 16}$ allele carries a mis-sense amino acid substitution in the first PDZ domain (Wada et al., 2005). In Ilk mutants, FBM and octavolateralis efferent (OLe) neurons failed to migrate out of $r 4$. This line was provided by Dr. Hitoshi Okamoto (RIKEN, Japan).

\subsection{7 detour ( $\left.d \operatorname{tr}^{t s 269}\right)$}

The detour (dtr) locus encodes Gli1, a transcriptional activator in the hedgehog $(\mathrm{Hh})$ signaling pathway (Chandrasekhar et al., 1999). In $d t^{t s 269}$ mutants, branchiomotor neurons were greatly reduced in number (Vanderlaan et al., 2005). 
Vanderlaan G., Tyurina O, Karlstrom R. and Chandrasekhar, A. (2005)

Gli function is essential for motor neuron induction in zebrafish.

Developmental Biology: 282: 550-570.

\subsection{8 nacre $\left(\operatorname{nac}^{w 2}\right)$}

The nacre (nac) locus encodes a transcription factor related to microphthalmia (Mitf) (Hodgkinson et al., 1993), a gene required for the development of eye and crest pigment cells in mouse. The nac ${ }^{22}$ allele lacked melanophores throughout embryonic stages to adulthood (Lister et al., 1999), except in the retinal pigment epithelium.

\subsection{Genotyping Mutant Lines}

\subsection{1 off limits (oltrw689)}

The primer used for genotyping:

Fwd: 5'- C G C A G A C A T A G C A A C A C T G G-3'

Rev: 5'- C T G C A T T T C G T T G C C T TG T A C C T-3'

The PCR products are run on 1\% MetaPhor agarose (Lonza, Catalog \# 50181) gel for 50 mins at 72 volts to separate the bands. PCR amplified a $517 \mathrm{bp}$ product from the 3 ' terminal region of the $f z 3 a$ gene Forward primer was used for sequencing.

The PCR settings used are as follow: 
$94^{\circ} \mathrm{C}: 2 \min$

$94^{\circ} \mathrm{C}: 30 \mathrm{sec}$

$54^{\circ} \mathrm{C}: 30 \mathrm{sec}$

$72^{\circ} \mathrm{C}: 20 \mathrm{sec}$

$X 40$ cycles

$72^{\circ} \mathrm{C}: 10 \mathrm{~min}$

$4^{\circ} \mathrm{C}$ Hold

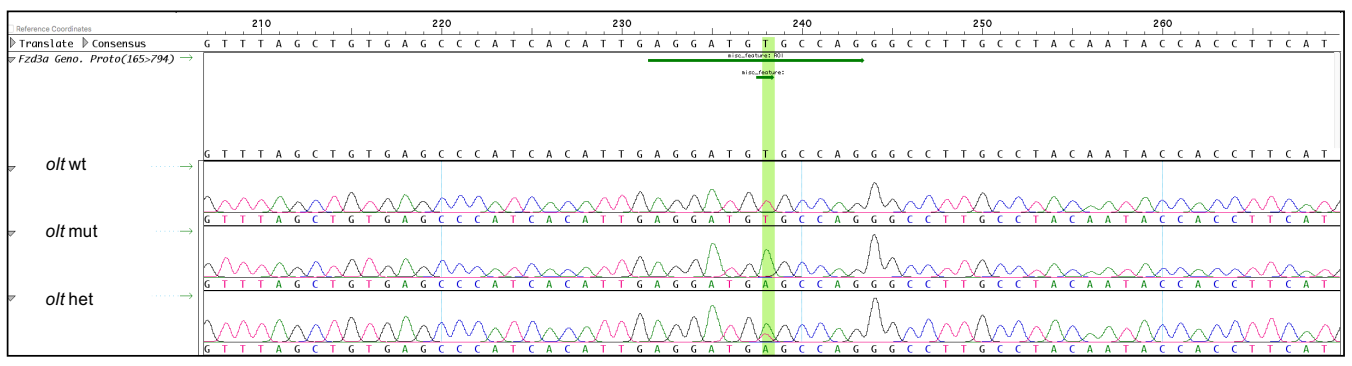

\subsection{2 landlocked (II/ $\left.k^{r 16}\right)$}

The following primers are used for genotyping:

Fwd: 5'-G A A G G C T G C T G G T T G G G A C G - 3'

Rev: 5'-G C G T G C A T T G C C T A C T G G C A T G- 3'

The PCR products are run on $2 \%$ MetaPhor agarose (Lonza, Catalog \# 50181) gel for $40-60$ mins at 72 volts to separate the bands.

The scrb1 gene is encoded by 45 exons that span $120 \mathrm{~kb}$ in the genome. Rev primer was used for sequencing.

The PCR settings used are as follow: 
$94^{\circ} \mathrm{C}: 2 \min$

$94^{\circ} \mathrm{C}: 30 \mathrm{sec}$

$57.5^{\circ} \mathrm{C}: 30 \mathrm{sec}$

$72^{\circ} \mathrm{C}: 20 \mathrm{sec}$

$72^{\circ} \mathrm{C}: 10 \mathrm{~min}$

$4^{\circ} \mathrm{C}$ Hold

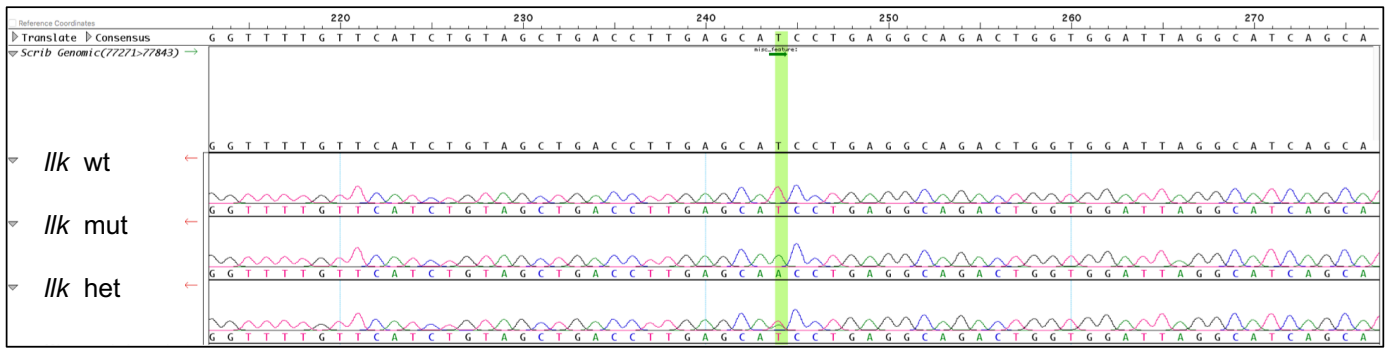

\subsection{Alcian Blue Staining}

Embryos were fixed in $4 \%$ paraformaldehyde overnight at $4^{\circ} \mathrm{C}$.

Embryos were washed $2 \times 5$ minutes in PBST and dehydrated through $50 \%$ $\mathrm{MeOH} / \mathrm{PBST}$ for 1 hour and into $100 \% \mathrm{MeOH}$ for 2 hours. $\mathrm{MeOH}$ was removed and embryos were placed in $0.15 \%$ Alcian Blue (Sigma) solution overnight at room temperature on shaker. Embryos were rinsed with $100 \%$ EtOH 2-4x quickly (until no blue color can be detected in the solution). Embryos were rehydrated through 50\% EtOH/PBST for 30 minutes and into $100 \%$ PBS for another 30 mins. Embryos were digested in $0.05 \%$ Trypsin in 1xPBS at room temperature for 2 hours. Embryos were washed 
$2 \times 5$ minutes each with PBST until bubbles were cleared. Embryos were bleached in $3 \% \mathrm{H} 2 \mathrm{O} 2,1 \% \mathrm{KOH}$ and $1 \% \mathrm{KOH}$ at room temperature until desired staining hue. Embryos were stored in $80 \%$ glycerol/0.1\% Tween at $4{ }^{\circ} \mathrm{C}$ or in $4 \%$ PFA.

\section{Solution Recipes}

$100 \mathrm{~mL}$ Alcian Blue Stock

0.15\% Alcian Blue

0.15g (Sigma \#A-5268 Alcian Blue 8 GX)

$50 \% \mathrm{EtOH}$ $50 \mathrm{~mL} 100 \% \mathrm{EtOH}$

$0.1 \mathrm{M} \mathrm{HCl}$ $860 \mu \mathrm{L} 36 \% \mathrm{HCl}$

$\mathrm{pH} \sim 1$ $49 \mathrm{~mL} \mathrm{dH_{2 } \mathrm { O }}$

PBST (50ml)

$50 \mathrm{ml} 2 \mathrm{X}$ PBS

$50 \mu \mathrm{l} 100 \%$ Tween-20

$\underline{50 \% \mathrm{MeOH} / \mathrm{PBST}}$

$20 \mathrm{ml} \mathrm{MeOH}$

$20 \mathrm{ml} \mathrm{PBST}$

$\underline{\text { Trypsin }(50 \mathrm{ml})}$

$498 \mathrm{ml}$ Trypsin

$2 \mathrm{ml} 1 \mathrm{x}$ PBS

\subsection{Presynaptic Neuromuscular Staining (SV2a)}


Larvae were fixed in 4\% paraformaldehyde in PBS for at least 12 hours (overnight) at $4^{\circ} \mathrm{C}$, washed in PBS $3 \times 5$ minutes, and treated with $0.25 \%$ trypsin in PBS on ice. Treatment times increased with age (15-20 min for $6 \mathrm{dpf}$ zebrafish, 10-15 min for $5 \mathrm{dpf}$ zebrafish, 5-7 min for $4 \mathrm{dpf}$ zebrafish - the range of times must be optimized empirically for each new batch of trypsin). Larvae were washed in PBS $4 \times 5$ minutes, blocked in IB (1 \% w/v BSA, $0.5 \% \mathrm{v} / \mathrm{v}$ Triton, and $1 \% \mathrm{v} / \mathrm{v}$ DMSO in PBS) containing $1 \%$ horse serum (Sigma) for $6-8$ hours at $4^{\circ} \mathrm{C}$, and incubated in primary antibody in PBST (PBS, $1 \%$ Triton) for 2 days at $4^{\circ} \mathrm{C}$. Larvae were washed for at least $5 \mathrm{x}$ for 1 hour in PBST then incubated in the dark (covered with aluminum foil) overnight or for 8-12 hours in fluorescent secondary antibody diluted in 1:500 in IB containing horse serum. Larvae were washed 3x 30 minutes in PBST then mounted and imaged immediately or fixed overnight in $4 \%$ paraformaldehyde. For mounting, larvae were washed $4 \times 5$ minutes with PBS, taken through a glycerol series $(25 \%$, $50 \%, 70 \%$ glycerol) and mounted in $70 \%$ glycerol. Larvae are kept in the dark at all times to minimize fading of the fluorescent signal.

\subsubsection{Antibody Concentration}

- Rabbit anti-GFP: 1:2000 (Invitrogen)

- Mouse a-SV2, 1:200 dilution (Developmental Studies Hybridoma Bank) 
- Goat anti-mouse Alexa Flour 568: 1:500 (Life technologies)

- chicken anti-rabbit Alexa Fluor 488 Mouse 488: 1:500 (Invitrogen)

\subsection{Postsynaptic Neuromuscular Staining ( $\alpha-B T X)$}

Larvae were incubated in 15\% DMSO in embryo medium (E3) and Alexa 594-conjugated $\alpha$-bungarotoxin ( $\alpha-B T X)(10 \mu \mathrm{g} / \mathrm{ml}$; Invitrogen) in an ice bath for 30 minutes, washed $4 \times 5$ minutes with cold PBS, mounted in $0.2 \%$ agarose in E3 and imaged immediately.

\subsection{Calcium Imaging}

Larvae (7 dpf) expressing GCaMP6s in branchiomotor neurons were paralyzed by injecting with $4.6 \mathrm{ng}$ of alpha-bungarotoxin (Invitrogen) at a concentration of $0.92 \mathrm{ng} / \mathrm{nl}$ in Danieau buffer (Alomone Labs) 1 hour before imaging, mounted dorsally in $0.2 \%$ agarose in $\mathrm{E} 3$, and covered with E3 medium. Larvae were imaged using Leica TCS SP8 MP inverted spectral confocal microscope with a $20 x / 0.75$ water immersion objective at 2 frames per second (fps) for 5 minutes (600 frames total). Images were analyzed using Fiji (NIH) and regions-of-interest (ROIs) were drawn around FBM neurons and trigeminal neurons based on their locations in the hindbrain. Background subtraction was performed on every frame in the same time series as FBM neurons and trigeminal neurons ROI by drawing an $\mathrm{ROI}$ in a region of the hindbrain lacking GCaMP-expressing 
cells, yielding the background-corrected trace. The $\mathrm{Ca}^{2+}$ spike events were counted from the traces and manually validated by two observers independently watching the time-lapse recordings.

\subsection{Confocal Imaging}

Larvae are anesthetized with tricaine, $(10 \mu \mathrm{g} / \mathrm{ml}$; Western Chemical Inc) and Z-stack images (1 $1 \mu \mathrm{m}$ thickness, $15-25$ slices) were taken of laterally-mounted larvae with Leica TCP SP8 MP confocal microscope at 20x magnification. Leica Application Suite X (LAS X) software was used to generate a 3D image from the stacks of confocal images. Images are analyzed LAS X or Fuji (NIH).

\subsection{Measurement of Axon morphologies Using LAS X Software}

The data stacks were collected as stated in Section 2.8 and placed in the "axon analysis" folder.

Follow these steps to trace and measure axon lengths in the 3D stacks.

i) Select the z-stack LIF data file in the LAS X.

ii) Delete the bright field channel.

iii) Click the "Process" tab -> Process Tools -> Crop.

iv) Two channel buttons are below the image: $\mathrm{Ch} 1$ and $\mathrm{Ch} 2$. Uncheck the Ch1 button and click "Apply". LAS X software creates a new stack only 
with fluorescent channel $\mathrm{Ch} 2$. Note that the original data set will be not altered.

v) Select the newly created channel z-stack stack and click on the 3D button. This will open the z-stack in the 3D mode.

vi) Click on the "Analysis" tab and open the 3D Image Analysis module.

vii) Start the image analysis pipeline by selecting the step 1 - Select Images to Measure.

viii) Select "Measurements" tab -> Tools -> polygon.

ix) Start measurement of main and branching axons by rotating 3D Image consistently, and adjust the polygon tool to ensure it is on the surface of the axons.

x) Exclude processing "sequences" 2-6

xi) Select Step 9 - "Measurements". Result table with the measurements is created.

xii) Export the data table as an Excel spreadsheet file.

xiii) Save it as a configuration in 3D.

\subsection{NMJ Measurements on Jaw Muscles Using}

\section{LAS X Software}

The data stacks were collected as stated in Section 2.8 and placed in the "BTX-post synaptic analysis" folder.

Follow these steps to generate the jaw muscle masks and measure the 
volumes of the stained structures on the muscles.

i) Select the z-stack LIF data file in the LAS X.

ii) Delete the bright field channel.

iii) Click the "Process" -> Process Tools -> Crop.

iv) Three channel buttons are below the image: $\mathrm{Ch} 1, \mathrm{Ch} 2$ and $\mathrm{Ch} 3$.

Uncheck the Ch3 button and click "Apply". LAS X software creates a new stack only with fluorescent channels $\mathrm{Ch} 1$ and Ch2. Note that the original data set will be not altered.

v) Select the newly created 2-channel z-stack stack and click on the 3D button. This will open the z-stack in the 3D mode.

vi) Click on the "Analysis" tab and open the 3D Image Analysis module.

On the toolbar select "Auto Range". In the analysis panel select "Multi Channel".

vii) Start the image analysis pipeline by selecting the step 1 - Select Images to Measure.

viii) In Channel Mappings click the "Append" button twice to create two populations of data: Population 1 and Population 2. "Acquisition" column of Channel Mappings assign Ch 2 (neuromuscular synapses, red) to the Population 1 and Ch 1 (GFP-labeled muscles, green) to the Population 2. Rename the Population 1 as "NMJ" and the Population 2 as "Muscles". In the "Stack Reduction" field and select "No Reduction (3D)". 
ix) Step 2- "Image Processing Pre-Filter". Population 1 data (NMJ) will be not filtered, "2 - Image Processing Pre-Filter" field is set to "No Filter". Click on the "Population 2" tab and select the "Remove noise - median" filter. Select filter size 2. Check the box "Filter Active" to see the effect of the filter on image. Keep the Filter Active checked.

x) Step 3- "Adjust Threshold" and select the NMJ channel. In this step carefully select the threshold objects. All objects with intensities above the threshold value will be measured and objects with intensities below the threshold will be considered "background". In the "Adjust Threshold" section set the thresholding to "Maximum Entropy". Set the threshold selector to ">" - all features at or above the threshold value will be included. Set "Tolerance" to 1. Check "Show Binary" and "Apply Automatically" checkboxes. The "Show Binary" (original z-stack image) allow going back and forth between the threshold intensity image and the binary image. Adjust the threshold value to the point when all objects in the NMJ channel are accurately and precisely defined. Note the threshold value range for 4-5 images and apply it to the NMJ channel of all data sets after. Click at the "Muscle" tab and use the same steps to threshold the image of muscles.

xi) Step 4 - "Binary Processing Pre-Filter". Select the "NMJ" channel and exclude it from this processing step by selecting an " $X$ " in the step 4 of the analysis pipeline. While still in the step 4, select the "Muscles" channel. In the "Binary Processing Pre-Filter" window, select the "Discard details - 
Open" morphological filter. Set size to 2 and enable 'Filter Active" checkbox. Do not enable the "Reconstruct" checkbox.

xii) Exclude the step 5 "Binary Operations" from the pipeline for both channels.

xiii) Step 6 - "Binary Image Editing". Select the NMJ channel and exclude the Binary Image Editing from the pipeline. Select the Muscles channel. In the Binary Image Editing window below the pipeline panel select the "Draw Straight Lines" method. Enable "Erase". Select the Line editing tool and set line width to 4 . In the binary image window draw the lines between the muscles into groups and left and right segments (ima/p, amR, amL, ihR, ihL, hhR, hhL, ) click the "Apply" button to divide the muscles. Color code divided muscle.

xiv) Exclude the step 7 "Measure Frame" for both channels.

xv) Step 8 "Reference Mask" and select the "Muscle" channel. In the window "Reference Mask" mark, Mask: "Muscles" and Mode: "Object count, Intensity, Area". Enable options "Count, Intensity, Area" in the NMJ image channel. Click on the "NMJ" channel tab. The image panel shows the binary image of the NMJ channel, the other panel shows the NMJ channel with overlaid measurement mask (reference mask).

xvi) Step 9 - "Measurements". Result table with the measurements is created. Four data tables are presented: Object Details (shows individual object features), Object Summary (object statistics), Image Measurements 
(summary of all objects within the measure frame), Image Summary (statistics of measurements shown in the Image Measurements window). Set Label to "Numbers" and set Mode to "All Features". Export the data table as an Excel spreadsheet file. Save it as a configuration in 3D.

\subsection{Food Intake Assay}

E3 medium without methylene blue $(5 \mathrm{ml})$ was placed in each well (feeding chamber) of a 6-well plate (Falcon, 08-722-1B). About 15-20 7 dpf larvae - were transferred to a $2 \mathrm{ml}$ centrifuge tube and the total volume was adjusted to $2 \mathrm{ml}$ before transferring the larvae to the feeding chamber. $2 \mathrm{ml}$ of E3 medium was removed from the well for a final volume of $5 \mathrm{ml}$ in each feeding chamber. Larvae were acclimated to the feeding chamber for 1 hour in the L/D $28.5^{\circ} \mathrm{C}$ incubator then $2 \mathrm{mg}$ of fluorescent larval food (Section 2.11.1) was mixed well with $1 \mathrm{ml}$ of E3 medium and added to feeding chamber. The 6-well plates were placed in the LD incubator for 3 hours for optimal larval feeding time (Allen et al., 2017), then $0.4 \%$ tricaine (Western Chemical Inc) was added to each chamber to paralyze the larvae and end feeding. Larvae were transferred to a petri dish containing E3 + tricaine and rinsed gently to remove fluorescent food particles adhering to the larvae. Larvae were positioned laterally in the multi-lane agar mold and submerged in 3\% methylcellulose. Larvae were assigned feeding scores ranging from 0-3: a feeding score of ' 0 ' 
corresponded to larvae with no fluorescent signal within the gut, while a score of ' 3 ' corresponded to fluorescence throughout the gut. Only food in the larval gut determined the feeding score (food past the stomach was noted but did not count toward score). Scoring was done in blinded fashion.

\begin{tabular}{|l|l|}
\hline Feeding Score & Description \\
\hline Feeding Score $=0$ & $\begin{array}{l}\text { Empty gut, no visible fluorescent } \\
\text { within gut }\end{array}$ \\
\hline Feeding Score $=1$ & $\begin{array}{l}\text { Low }- \text { very little. Roughly up to } \\
<25 \% \text { of gut }\end{array}$ \\
\hline Feeding Score $=2$ & $\begin{array}{l}\text { Medium - Some fluorescence in } \\
\text { gut. Roughly up to } 25-50 \% \text { of gut }\end{array}$ \\
\hline Feeding Score $=3$ & High - Roughly half of gut or more \\
\hline
\end{tabular}

\subsubsection{Preparation of Fluorescent Larval food}

Mix $100 \mathrm{mg}$ of larval fish food (Zeigler $<100$ microns) with $50 \mu \mathrm{l}$ of deionized water in a weigh boat. Make a hole in the food slurry and add $150 \mu \mathrm{l}$ Yellow-Green fluorescent microsphere solution (Molecular Probes/ThermoFisher; vortex microsphere solution well before adding) in low light conditions. Mix gently for 5 minutes to form a paste. Spread paste with spatula as a thin and even layer over a watch glass or microscope slide. Place in drying box (foil lined cardboard box) and allow to dry in the 
dark. Scrape dried paste off the glass surface and crush finely between two pieces of weighing paper. Store fluorescent larval food powder protected from light at $4^{\circ} \mathrm{C}$.

\subsubsection{Preparation of Multi-Lane Agar Plate}

Dissolve 1.2 grams of agarose (Gold Biotechnology) in $100 \mathrm{ml}$ of E3 (no methylene blue) in a glass flask. Microwave gently in 10 second increments until agarose is fully dissolved. Pour agarose solution into 100 $\mathrm{mm}$ petri dish to $\sim 1 / 3$ height. Place six-lane mold $(4 \times 6.5 \mathrm{~cm})$ into agarose solution until it solidifies at room temperature then use tungsten wire to remove the molds.

\subsubsection{Preparation of 3\% Methyl Cellulose With $0.016 \%$ Tricaine}

Mix $24 \mathrm{ml}$ of E3 with $1 \mathrm{ml}$ of $0.4 \%$ tricaine stock in a $50 \mathrm{ml}$ Falcon tube. Add $0.75 \mathrm{~g}$ of methyl cellulose powder (Sigma) and vortex mixture for 5-10 min breaking up large particles until it turns viscous with lots of bubbles. Parafilm the tube and continue mixing with gentle rocking at $4^{\circ} \mathrm{C}$ for at least 48 hours until solution acquires a smooth viscous consistency with few bubbles. Store at $4^{\circ} \mathrm{C}$ and keep on ice during use. 


\subsubsection{Preparation of Tricaine Wash}

Mix $48 \mathrm{ml}$ of E3 medium with $2 \mathrm{ml}$ of $0.4 \%$ tricaine stock in a $50 \mathrm{ml}$ Falcon tube and store at $4^{\circ} \mathrm{C}$.

\subsection{Jaw (Gape) Movement Assay}

Larvae were tested by transferring them one at a time with a glass pipette into the multi-lane agar plate, making sure to minimize liquid transfer. A drop of melted $2 \%$ LMP agarose solution (stored at $37^{\circ} \mathrm{C}$ during use) was placed over the larva, which was carefully positioned to achieve an ideal lateral orientation. A few drops of $1.2 \%$ agarose were added to cover the trunk of the larva. Excess agarose was removed from the head region to enable the jaw to move freely, and E3 medium was added to the depression (lane). The larva was recorded one minute after jaw movement begins, but was discarded if no jaw movement was evident after 10 min. Each larva was tested only once.

\subsubsection{Preparation of LMP agarose}

Dissolve $2 \mathrm{gm}$ of LMP agarose in $100 \mathrm{ml}$ of $\mathrm{E} 3$ medium in a glass flask. Microwave at low power with frequent swirling until agarose solution is fully dissolved. Aliquot into $15 \mathrm{ml}$ Falcon tubes (10 $\mathrm{ml}$ per tube) and 
store at $4^{\circ} \mathrm{C}$. Agarose should be microwaved gently before use and placed in $37^{\circ} \mathrm{C}$ bath while prepping for experiment.

\subsection{Swimming Assay}

Larvae at $6 \mathrm{dpf}$ were placed in individual wells of a 24 -well plate and observed at room temperature $\left(21-22^{\circ} \mathrm{C}\right)$ using the DanioVision system and EthoVision XT 8.0 locomotion tracking software (Noldus). Larvae were allowed to acclimate to the dark for 30 minutes. Larvae were analyzed using EthoVision to measure distance moved and moving duration. Swimming analysis was performed at Georgia Southern University in Dr. Vinoth Sittaramane's lab. 


\section{CHAPTER 3: DISSECTING BRANCHIOMOTOR}

\section{NEURON CIRCUITS IN ZEBRAFISH TOWARD HIGH- \\ THROUGHPUT AUTOMATED ANALYSIS OF JAW MOVEMENTS}

Yasmin M. Kassim ${ }^{1 *}$, Noor M. Al-Shakarji1 ${ }^{1}$ Emilia Asante ${ }^{2}$, Anand

Chandrasekhar $^{2}$, K. Palaniappan ${ }^{1}$

${ }^{1}$ Computational Imaging and VisAnalysis (CIVA) Lab

Department of Electrical Engineering and Computer Science

${ }^{2}$ Division of Biological Sciences and Bond Life Sciences Center

University of Missouri-Columbia, Columbia, MO 65211 USA

*Modified for Thesis: Y. M. Kassim, N. Al-Shakarji, E. Asante, A.

Chandrasekhar and K. Palaniappan, "Dissecting branchiomotor neuron circuits in zebrafish toward high-throughput automated analysis of jaw movements," 2018 IEEE 15th International Symposium on Biomedical Imaging (ISBI 2018), Washington, DC, 2018, pp. 943-947, doi:

10.1109/ISBI.2018.8363726.

\subsection{ABSTRACT}


We use the zebrafish model to understand brain circuits containing branchiomotor neurons that control jaw movements in vertebrates. In one approach, we compare circuit output, evaluated by dynamic analysis of jaw movement from video recordings, between wildtype zebrafish and mutants with specific circuit defects. While such analyses have been informative, they require extensive manual annotation. Thus, we developed an automated image analysis system to extract motion features from microscopy video in order to facilitate rapid and accurate detection of jaw movement, termed gape. The approach uses a robust fusion process combining local optical flow fields with background subtraction motion modeling into a likelihood response map, followed by peak processing to reliably detect jaw movement. Performance on 24 labeled videos of zebrafish larvae across three age groups achieved performance of $97.2 \%$ recall, $93.5 \%$ precision and $98.7 \%$ accuracy. The automated system demonstrates high fidelity in jaw movement detection and can be expanded for high-throughput studies in neuroscience and pharmacology.

Index Terms - Zebrafish, branchiomotor circuit, behavior, video microscopy, optical flow, background subtraction, motion map

\subsection{Introduction and Jaw Motion Analysis}

Chewing and swallowing are essential motor functions involving the coordination of jaw and other pharyngeal muscles as well as associated 
neural networks that are controlled by branchiomotor neurons located in the brainstem (Chandrasekhar, 2004) (Guthrie, 2007). Defects in these motor functions (dysphagia) are often associated with neurological diseases including Parkinson's and Amyotrophic Lateral Sclerosis (ALS) (Jani and Gore, 2016) (Kane et al., 2011) (van Bruggen et al., 2014). Although the organization and development of the branchiomotor neurons have been studied extensively (Chandrasekhar, 2004) (Guthrie, 2007) (Lumsden and Krumlauf, 1996), the formation and functional outputs of the neural networks driving jaw muscle movement have received less attention. Imaging-based studies to understand organism level behaviors using the zebrafish model system are receiving increased attention (Cario et al., 2011) (Peravali et al., 2011) (Spomer et al., 2012) (Rinner et al., 2005) (Stern et al., 2011). Advances in computational neuroscience and high-throughput imaging to investigate phenomics of model systems such as zebrafish (Danio rerio) may provide insight into functional deficits such as chewing problems arising from aging and neurological disorders. In this study, we first analyzed jaw movement (gape) dynamics by videorecording zebrafish larvae using manual annotation and morphology analysis, as shown in Figure 3.1. Jaw movement results in the opening of the mouth, termed the gape, which was manually outlined in every movie frame. Gape area is a measure of the extent of mouth opening and becomes very robust and periodic in older larvae. 
We documented differences in gape frequency (rate of mouth opening) between wildtype larvae and a genetic mutant containing branchiomotor circuit defects. However, due to the bottleneck in manual annotations, these analyses were performed at low frame rates and for short durations. To address this issue, we created an image processing pipeline to facilitate automated high-throughput jaw movement analysis of videos captured at high frame rates and long duration. We combine two motion analysis methods, robust optical flow and background subtraction, using a unique robust fusion process that accurately detects gape or mouth opening events. Automated image analysis estimated gape frequencies that were very similar to those obtained manually.

Our workflow includes: (a) Analyzing zebrafish microscopy videos using two low level motion estimation methods including background subtraction and optical flow, (b) Robustly fusing these noisy motion estimates to produce a likelihood map that characterizes gape opening events, and (c) Quantitative assessment of our automated responses with annotations from expert biologists. Rest of the paper is organized as follows; section 2 describes zebrafish experimental system and motion analysis methods utilized in our tool. Section 2.2 provides the detailed steps in our robust fusion process. Section 3 describes the evaluation metrics. Section 4 provides the experimental results with comparison to manual ground truth traces then conclusions. 


\subsection{Deformable Jaw Motion Analysis}

Our automated image processing pipeline using deformable motion estimation is shown in Figure 3.1. The data input to the pipeline are microscopy video frames and the output is a gape trace plot that is used to determine zebrafish jaw frequency using a peak identification process. Multiple motion estimation combined with robust fusion is used to reliably merge motion fields and separate global from local motions. Our automated output has multiple responses in successive frames due to the fact that a fish can take 3 to 4 frames to open its mouth. Therefore, we have implemented a peak identification process as a post processing step that can detect the max response over successive frames while considering the possibility of fast opening, closing and reopening, which occurs in wild type zebrafish more frequently than mutants.

\subsubsection{Multiple Motion Models of Jaw Movements}

In order to accurately track the zebrafish larvae jaw movement behavior we apply a multiple motion estimation-based approach followed by fusion. Optical flow estimation is a widely used method for motion detection and filtering. In this paper, we used the approach proposed in (Sun et al., 2010) for robust motion flow estimation. It uses a vector median filter to de-noise the flow and preserves the energy in the final result on the image 
region, and set the pixel and derivative value of the pixel moving out of boundaries to zero; such features are useful when manual cropping is applied to the region of interest.

In addition to optical flow field estimates, we incorporated a background subtraction model to filter out responses generated by global animal movement within the cropped region. The role of global motion filtering is to isolate responses only in places where the jaw is undergoing articulated motion. For background subtraction, extensive parameter sweeping was tested to identify a threshold of 0.3 with the first 10 frames as the initial background model.

\subsubsection{Proposed Robust Fusion of Motion Fields}

Multiple motion fields are fused, assuming independent statistics of detection. The time varying optical flow fields are combined with background subtraction responses in those regions where the flow vectors are appropriately oriented, then we compute the fused gape likelihood response as the integrated value within the (cropped) jaw region using our unique robust fusion equation. The jaw region of interest can be identified using template methods or manually specified in the first frame. The cropped region usually has flow vectors in different directions due to unrelated fish muscle movement. These movements may result in large 
motion detections for the jaw region and lead to false positive gape responses. To mitigate this problem, we apply the robust motion fusion function based on Eq. 4 with the following characteristics:

1. Analyze the 2-D optical flow vectors $(u, v)$ in the mouth ROI to extract four directional motion maps.

2. Keep only one region (filtered region) that indicates the desired direction of the gape opening.

3. Compute the magnitude of the flow field within the jaw (cropped) region.

4. Mask the magnitude with the filtered region to get the magnitude only in the region of interest.

5. Fuse the masked magnitude with background subtraction for the cropped region to filter out noisy responses.

6. Compute the area for the fused region to generate a single variable gape response vs time plot.

\subsection{Quantitative Performance Evaluation}

The performance of the automated gape detection image processing pipeline was evaluated by comparison with manually labeled video frames referred to as ground-truth (GT). Three statistical measures were scored including recall (sensitivity), precision and accuracy defined as follows: 


$$
\begin{gathered}
\text { Recall }=\frac{T P}{T P+F N} \\
\text { Precision }=\frac{T P}{T P+F P} \\
\text { Accuracy }=\frac{T P+T N}{T P+F P+F N+T N}
\end{gathered}
$$

An additional quantity to assess accuracy of the automated system is to measure the absolute difference in gape counts,

Gape $_{\text {count }}=\mid G T_{\text {gapecount }}-$ Automated $_{\text {gapecount }} \mid$.

\subsection{Experimental Results and Discussion}

We started by testing whether jaw movements were affected in a zebrafish (Danio rerio) mutant where a subset of the BM neurons are mispositioned due to a failure in neuronal migration (Wada et al., 2006) (see Figure 3.4). While jaw movement was robust in mutants, gape frequency was much lower in a $7 \mathrm{dpf}$ mutant larva (blue trace, $4+/-1$ gapes/20 sec, $N=19$ larvae) compared to a wildtype larva (red trace, $14+/-3$ gapes/20 sec, $N=20$ larvae); see Figure 3.4 (bottom). This mutant line with mispositioned BM neurons exhibited reduced gape frequency. Our aim is to use highthroughput imaging methods to characterize gape frequency phenotypes of mutations and pharmacological treatments that affect the connectivity or 
function of the branchiomotor circuits, potentially leading to variations in jaw movement.

The imaging procedure for the dynamic analysis of jaw movements in zebrafish larvae is as follows: Zebrafish larvae between the ages of 3 to 9 days post-fertilization (dpf) were embedded in $2 \%$ low-melting point agarose (Sigma) dissolved in E3 embryo medium (Westerfield, 1995), with the jaw region able to move freely (see Figure 3.1). Jaw movements were imaged under brightfield with an Olympus SZX12 stereomicroscope (90X) fitted with a Retiga 2000 camera (QImaging, Inc.), and videos were recorded at 5 frames per second for 20 seconds. The 100 -frame movies were manually analyzed by tracing using Dynamic Image Analysis System (DIAS) software [12]. Jaw movement based on extent of mouth opening, termed the gape, was manually outlined in every movie frame and used as the labeled ground-truth.

Our pipeline was tested on 24 zebrafish microscopy videos at three different larval time points including 7 videos from $5 \mathrm{dpf}, 10$ videos from 7 dpf (5 wildtype and 5 mutant) and 7 videos from $9 \mathrm{dpf}$. These videos were selected by biologists to evaluate the efficiency of our pipeline. The performance of the automatic method was evaluated using recall, precision, accuracy, and absolute difference of counts compared to the gold standard manually labeled videos by biologists. Table 3.1 provides the average count and standard deviation for each age $(5,7$, or $9 \mathrm{dpf})$ category; the last row is the overall average and standard deviation across 
all videos. The overall recall of $97.2 \%$ is higher than the overall precision of $93.5 \%$ indicating that our method rarely misses an event, but has more false positives. The latter is due to uncontrolled deformable fish motion and background agarose medium fluid motion. Gape counts range between 4 and 25 while the maximum error in gape count is 3 (Figure 3.5, green bars). Figure 3.6 shows a temporal visualization where we superimposed our automated gape extraction results in red color compared to manual tracing results in blue color, and green dots marking jaw opening gape events. The automatic detections are well synchronized with the GT trace and gape events.

\subsection{CONCLUSIONS}

The long-term objective of our research are to examine how aging, disease and injury affect the functional organization and output of the branchiomotor circuits. In this paper, we describe an automated video microscopy tool for measuring jaw movement behavior, specifically gape or mouth opening, at the organism level across different developmental time points. Our method utilizes two motion analysis algorithms, optical flow, and background subtraction, and combines the motion estimates using a robust fusion process with peak detection using non-maximum suppression. The proposed fusion helps us to eliminate motion vectors in uninformative directions and to retain only a specific orientation correlated with the gape event within the selected ROI. Using this tool, we extracted 
gape frequency from 24 video sequences comprising 2400 frames of different individuals. The results are promising based on precision-recall performance measures and compare favorably with manual annotation. This tool will enable high-throughput dynamic analysis of the branchiomotor neural network output (i.e., jaw movement) in response to a broad spectrum of physical, chemical, and genetic factors. For future work, the tool will be enhanced to automatically locate the jaw ROI in video recordings, extract deformable motion parameters and identify morphological features not amenable to manual annotation in order to facilitate discovery of novel jaw dynamics of biological relevance, with potential translational applications to pharmacology, neurophysiology, and neuropsychiatry.

\subsection{ACKNOWLEDGMENTS}

Research supported by funds from the Bond Life Sciences Center and the University of Missouri (URC-17-078-n) to AC and partially by NIH R33EB00573 (KP). 


\subsection{FIGURES AND LEGENDS}

Figure 3. 1 Manual workflow for zebrafish jaw movement analysis

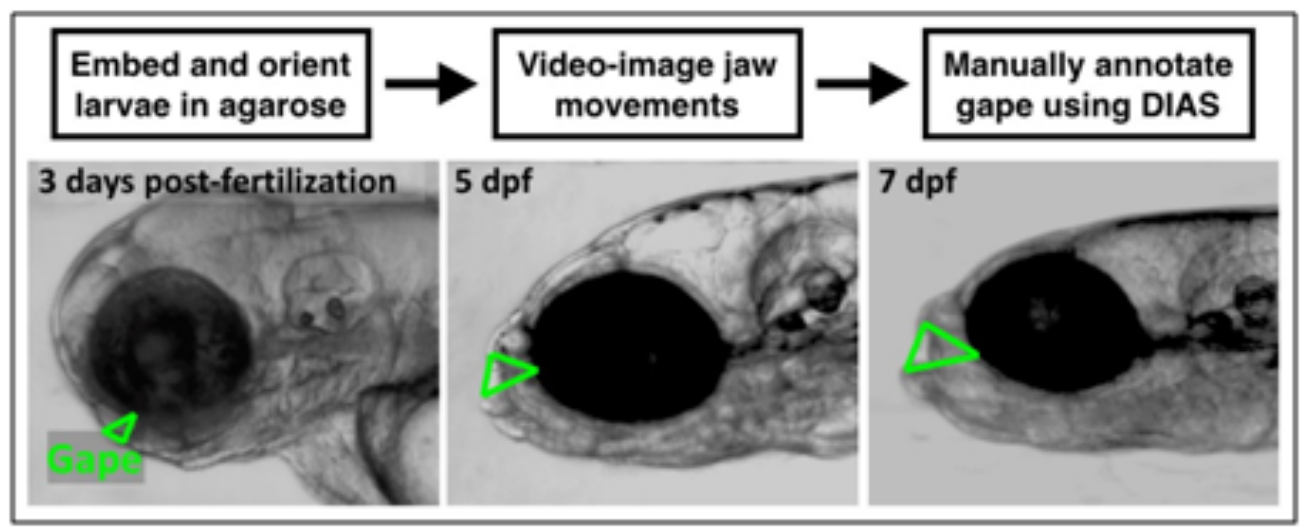


Figure 3.1 Manual workflow for zebrafish jaw movement analysis using DIAS (Soll et al., 2001). Gape feature (green) is outlined manually in individual frames of movies of $3 \mathrm{dpf}, 5 \mathrm{dpf}$, and $7 \mathrm{dpf}$ larvae. 
Figure 3.2 Branchiomotor neurons in $3 \mathrm{dpf}$ zebrafish larvae
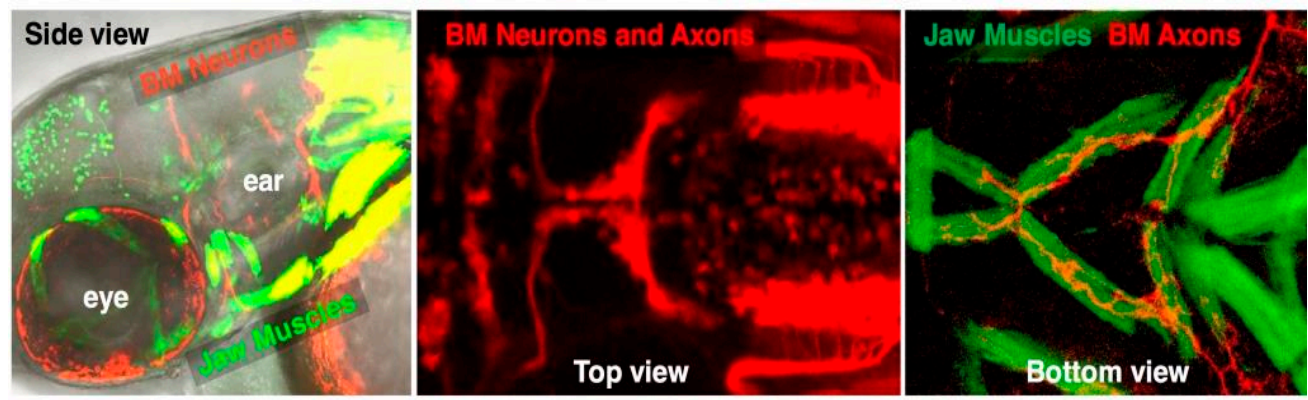
Figure 3.2 Side, top and bottom views of branchiomotor neurons and axons (red) and jaw and gill muscles (green) in 3 dpf zebrafish larvae. 
Figure 3.3 High-throughput image analytics pipeline

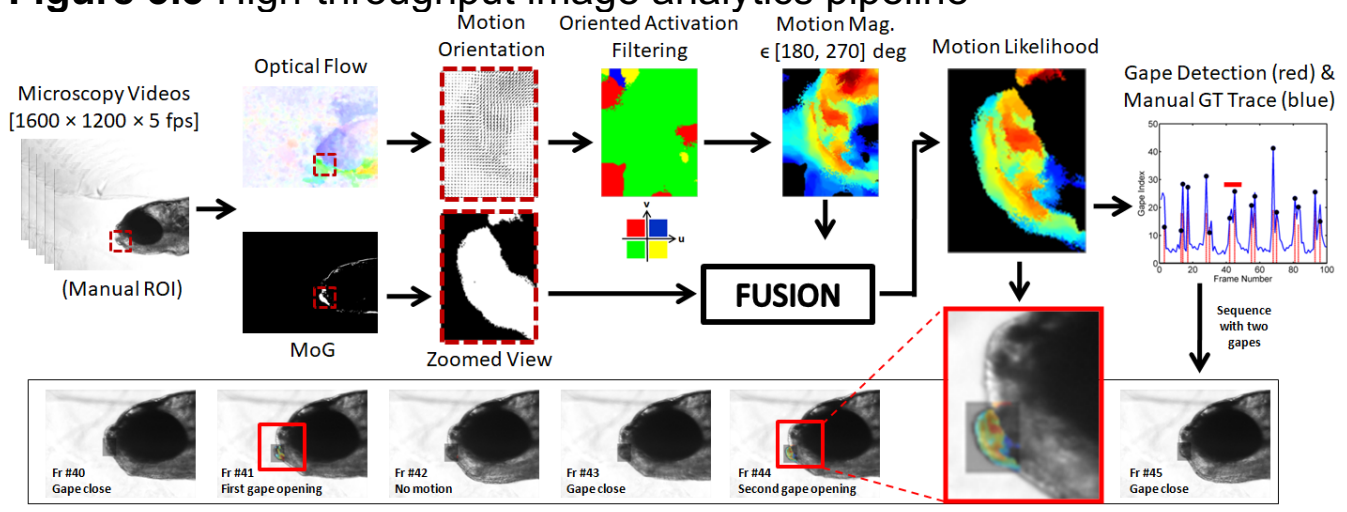


Figure 3.3 Throughput image analytics pipeline using motion estimation and fusion for zebrafish larval jaw movement and frequency analysis. The region of interest $(\mathrm{ROI})$ for gape analysis is currently manually specified in the first frame of the video. 
Figure 3.4 Mispositioned BM neurons in larval brain

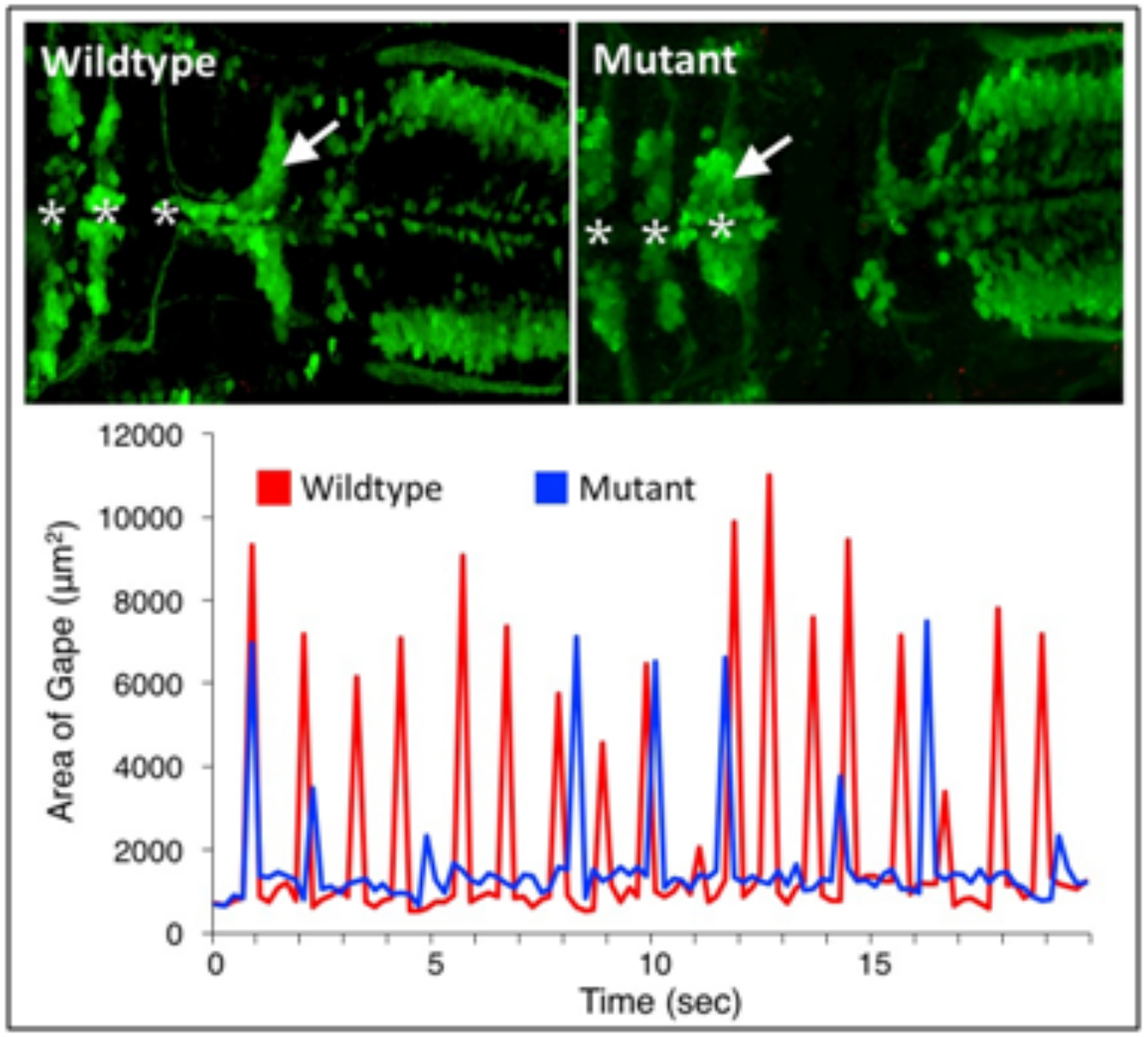


Figure 3.4 Top views of wildtype and mutant larval brains showing mispositioned BM neurons (arrow) in the mutant. The gape frequency (number of peaks per $20 \mathrm{sec}$ ) is reduced in the mutant larva (blue) compared to the wildtype (red). 
Figure 3.5 Plot of gape count vs video number

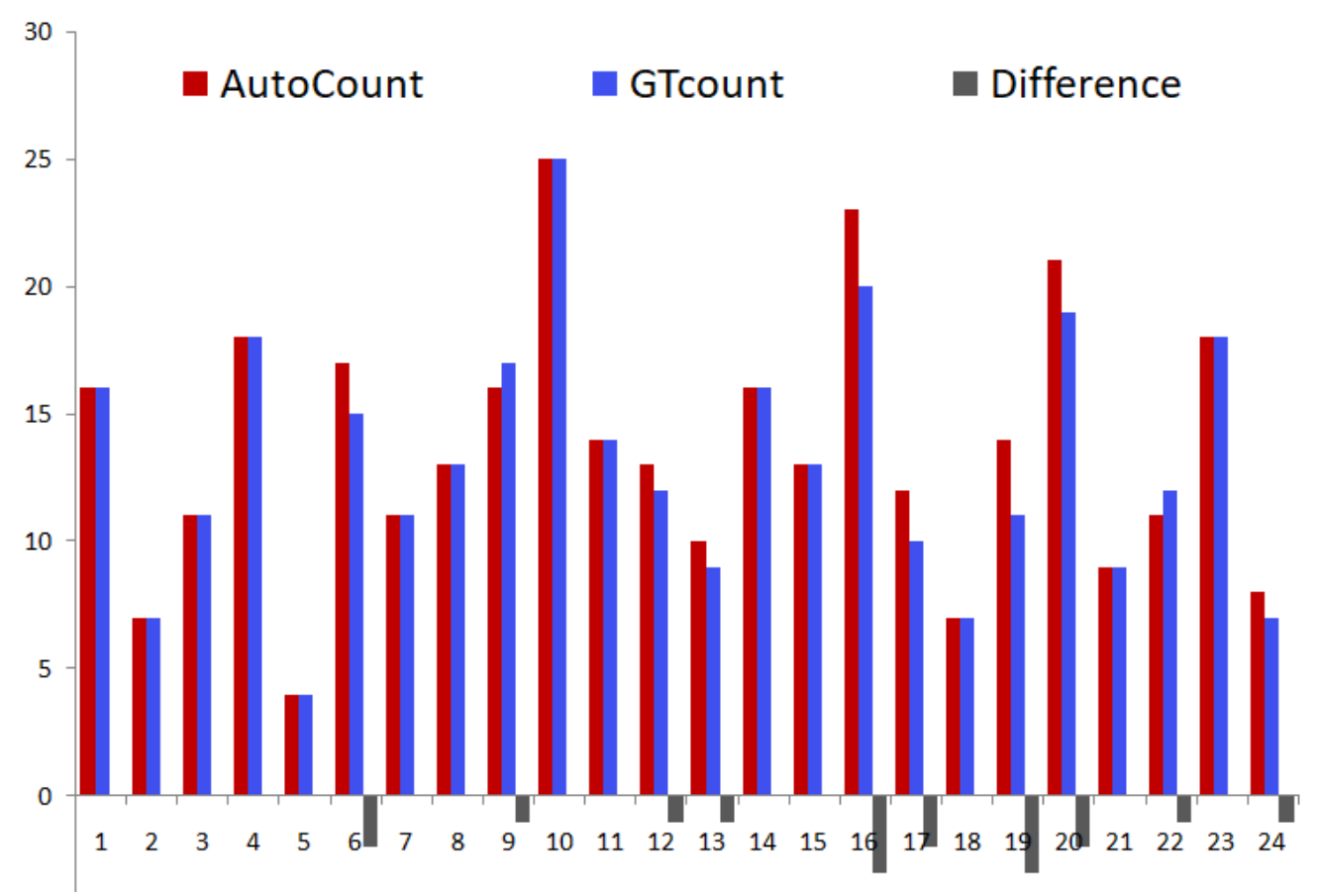


Figure 3.5 Plot of gape count vs video number showing that automated extraction of gape is reliable across 24 different sequences; gray color bar represents the difference in gape count between automated and manual results. 
Figure 3.6 Plot of gape area vs time

(a) 7-dpf Mutant 4

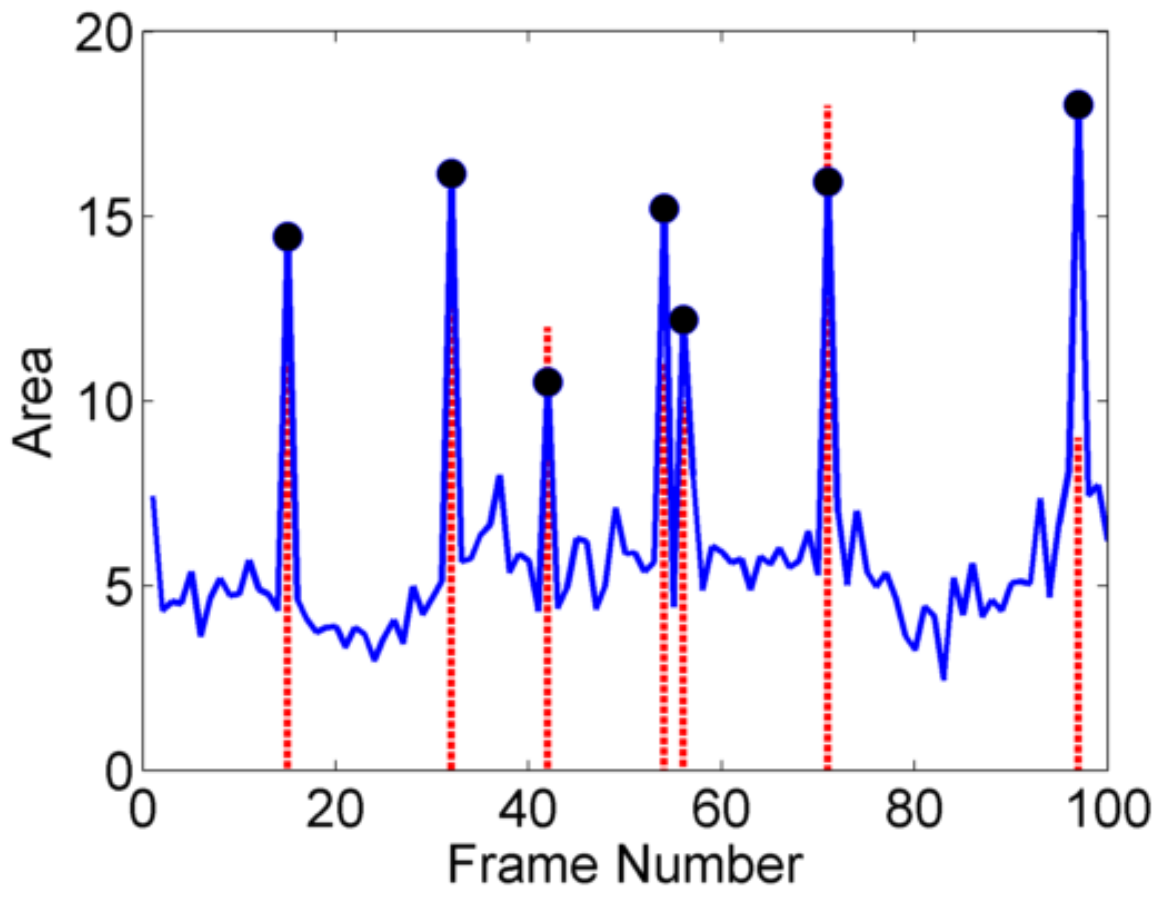

(b) 7-dpf Mutant 6

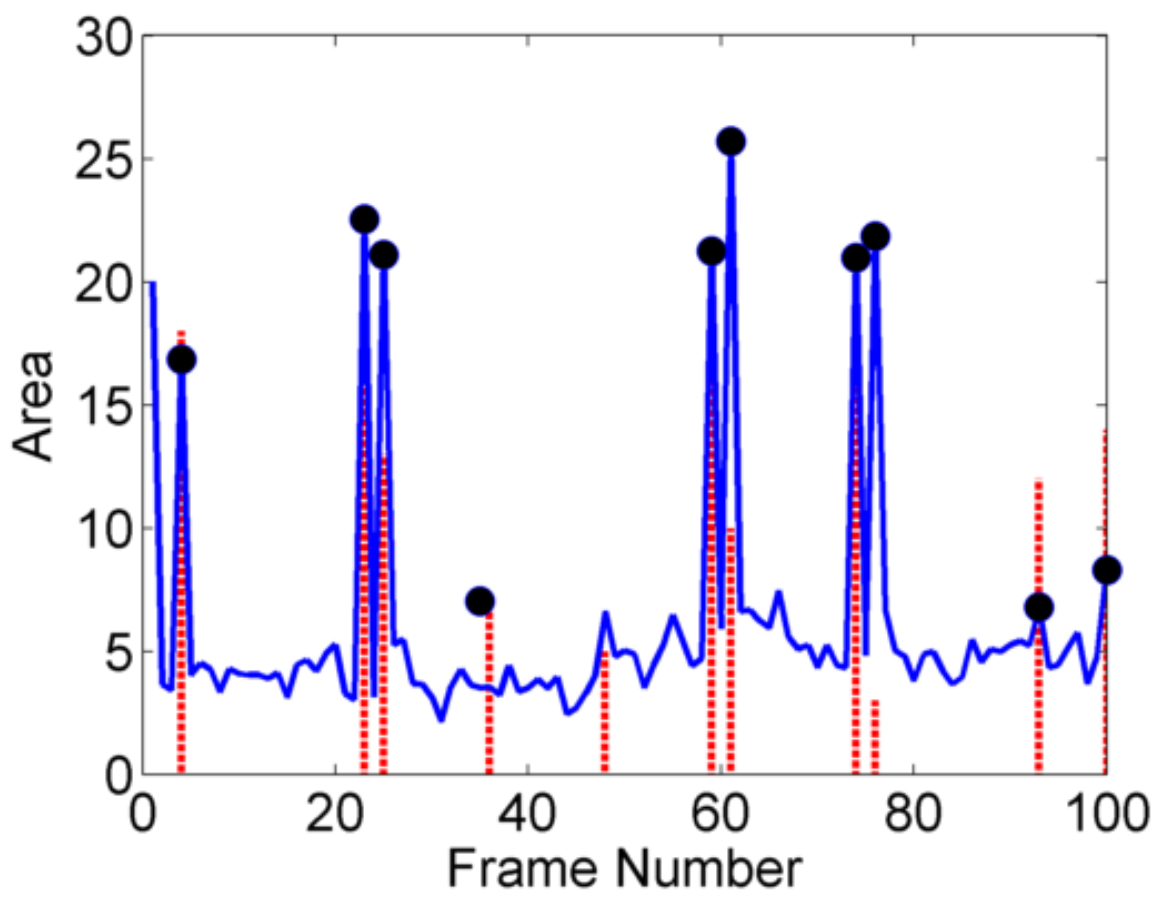


(c) 7-dpf Wildtype 5

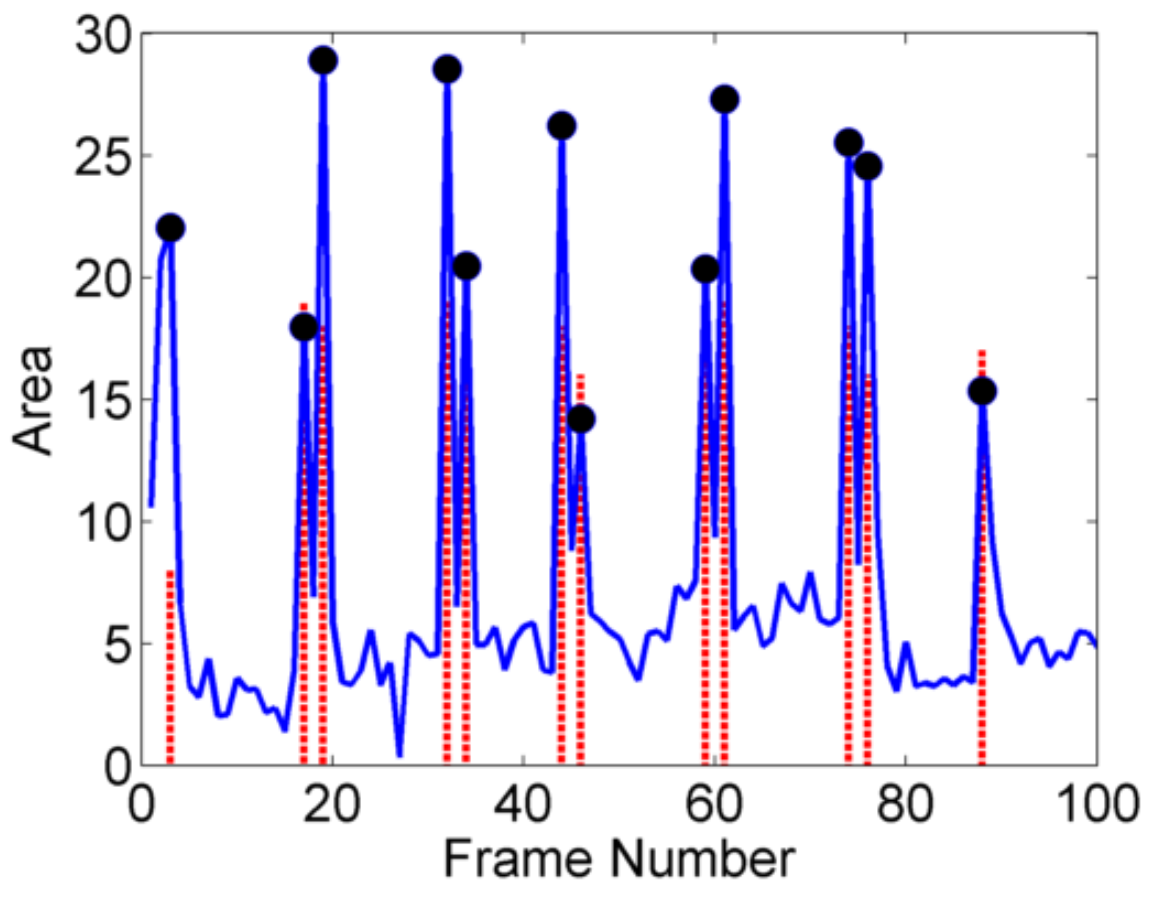

(d) 7-dpf Wildtype 10

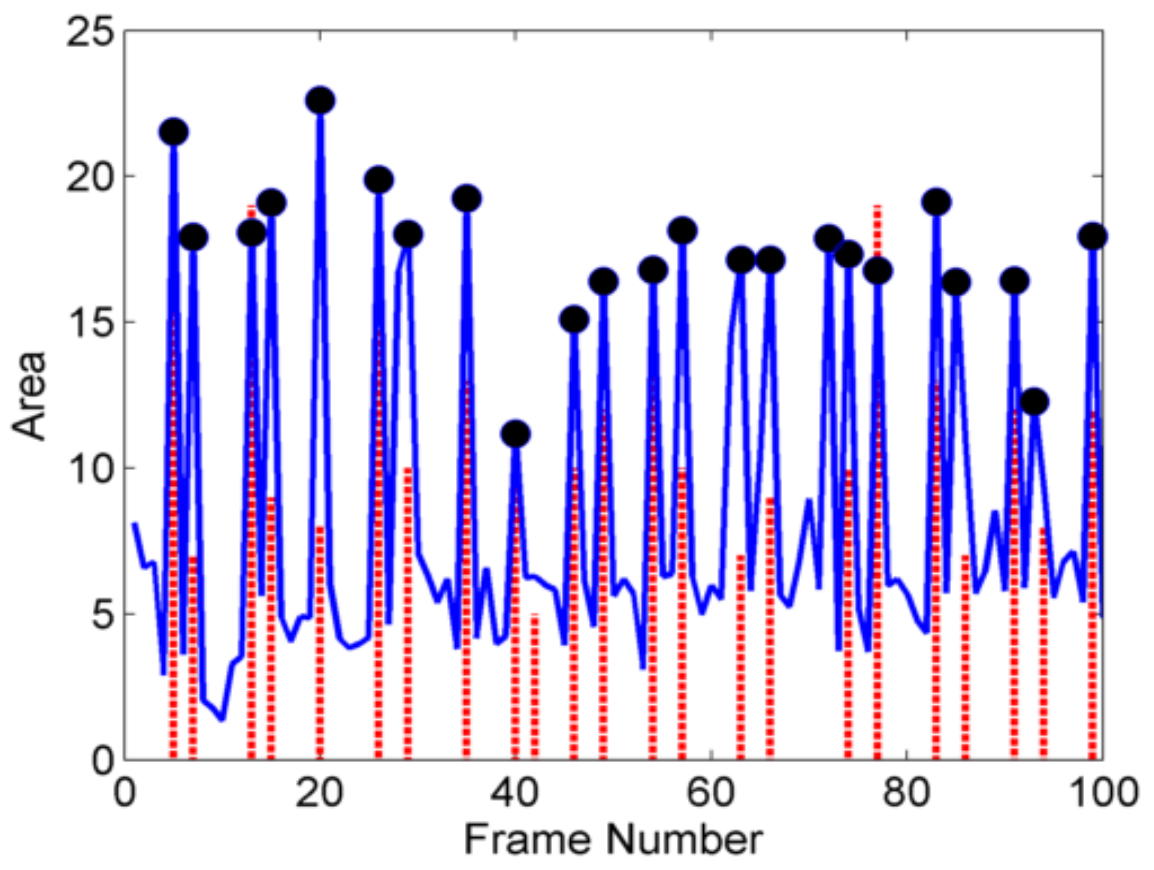


Figure 3.6 Plot of gape area vs time for two mutant and two wildtype gape plots that show automated results (red) super- imposed on biologist manual tracing using DIAS (blue) with ground-truth (GT) gape events marked as black dots. 


\subsection{TABLES}

Table 3.1

\begin{tabular}{|c|c|c|c|c|}
\hline Larval Age & $\begin{array}{c}\text { Number of } \\
\text { Videos }\end{array}$ & Recall \% & Precision \% & Accuracy \% \\
\hline $5 \mathrm{dpf}$ & 7 & $97.6 \pm 6.3$ & $92.4 \pm 8.1$ & $98.7 \pm 1.4$ \\
\hline $7 \mathrm{dpf}$ & 10 & $96.1 \pm 4.7$ & $95.4 \pm 4.5$ & $98.7 \pm 1.2$ \\
\hline $9 \mathrm{dpf}$ & 7 & $98.4 \pm 4.2$ & $91.8 \pm 8.5$ & $98.7 \pm 1.4$ \\
\hline Average & & $97.2 \pm 4.9$ & $93.5 \pm 6.8$ & $98.7 \pm 1.2$ \\
\hline
\end{tabular}

Table 3.1 Quantitative evaluation at the frame correspondence level for three different age groups of zebrafish larvae. 


\section{CHAPTER 4: DEFECTS IN NEURONAL POSITIONING IMPACT MOTOR CIRCUIT FUNCTION IN ZEBRAFISH}

\section{Submitted to Frontiers in Neural Circuits}

Emilia Asante ${ }^{1}$, Devynn Hummel ${ }^{1}$, Suman Gurung ${ }^{1,2}$, Yasmin M. Kassim³, Noor Al-Shakarji ${ }^{3}$, Kannappan Palaniappan ${ }^{3}$, Vinoth Sittaramane ${ }^{4}$, and Anand Chandrasekhar ${ }^{1}$

1, Division of Biological Sciences and Bond Life Sciences Center, University of Missouri, Columbia, MO 65211, USA.

2, Current address: Department of Pathology and Cell Biology, USF Health Heart Institute, University of South Florida, Tampa, FL 33602, USA.

3, Computational Imaging and VisAnalysis (CIVA) Lab Department of Electrical Engineering and Computer Science, University of Missouri, Columbia, MO 65211, USA.

4, Department of Biology, Georgia Southern University, Statesboro, GA 30458, USA.

Corresponding Author:

Dr. Anand Chandrasekhar 
Division of Biological Sciences

Room 340D Bond Life Sciences Center, 1201 Rollins St

University of Missouri, Columbia, MO 65211-7310

Tel: (573) 882-5166; Fax: (573) 884-9676

Email: AnandC@missouri.edu

Running Title: Abnormal Neuronal Positioning Affects Circuit Function in Zebrafish

Keywords: Jaw movement; Food intake; Behavior; Neuronal migration; Axon guidance; Neural circuits, Facial branchiomotor neuron; Zebrafish 


\subsection{ABSTRACT}

Precise positioning of neurons resulting from cell division and migration during development is critical for normal brain function. Disruption of neuronal migration_can cause a myriad of neurological disorders. To investigate the functional consequences of defective neuronal positioning on circuit function and behavior, we studied a zebrafish neuronal migration mutant off-limits/frizzled3a (olt/fz3a) where the facial branchiomotor (FBM) neurons fail to migrate out of their birthplace. We employed two behavioral assays to evaluate the functional output of the motor circuit involving the FBM neurons. Using a jaw movement assay, which measures the opening of the zebrafish jaw (gape), we observed a decrease in the frequency of gape events, but not their amplitude, in olt mutants. Consistent with this, using a larval feeding assay, we observed a decrease in food intake in olt mutants, indicating that the FBM circuit in mutants generates defective functional outputs. We tested several potential mechanisms that could underlie the defective functional output of the mutant FBM circuit. While $f z d 3 a$ is ubiquitously expressed in neural and non-neural tissues, jaw cartilage and muscle developed normally in olt mutants, and muscle function also appeared to be unaffected. Although FBM neurons were mispositioned in olt mutants, axon pathfinding to jaw muscles as assayed by axon lengths and branching patterns were unaffected. Moreover, a detailed analysis of structures labeled with presynaptic vesicle and postsynaptic receptor markers showed that the volumes of neuromuscular 
junctions established by FBM neurons were similar between wildtype siblings and olt mutants. Interestingly, FBM axons innervating the interhyoideus muscle were frequently defasciculated in olt mutants. Furthermore, GCaMP imaging revealed that mutant FBM neurons were less active than their wildtype counterparts. These data suggest that defective positioning of FBM neurons in olt mutants leads to defects in fasciculation and neuronal activity, potentially generating defective functional outputs. 


\subsection{INTRODUCTION}

The complex architecture of the vertebrate brain, especially the mammalian brain, is generated in part by the coordinated and orchestrated migration of neurons during development. Neuronal progenitors often migrate extensively using two modes to disperse throughout the central nervous system, radial migration and tangential migration (de Rouvroit and Goffinet, 2001; Marín et al., 2010; Sun et al., 2010). These events are critical developmental processes essential for precise neuronal positioning and establishing functional neural circuits (Gilmore and Herrup, 1997; Tau and Peterson, 2010). Failure of neuronal migration can lead to brain malformations associated with neurological conditions such as lissencephaly, epilepsy, and autism spectrum disorders (Copp and Harding, 1999; Gleeson and Walsh, 2000; Manent and LoTurco, 2013; Pan et al., 2019). In type 1 lissencephaly, which is characterized by a lack of gyrification, pyramidal projection neurons undergo incomplete radial migration resulting in the disruption of the cortical laminae and leading to poor motor function, seizure, and varying levels of intellectual disabilities (Kato and Dobyns, 2003; Moffat et al., 2015). However, the underlying mechanisms by which abnormal neuronal migration and positioning generate defects in circuit organization and behavior are poorly understood.

The motor circuits generated by the vertebrate facial branchiomotor (FBM) neurons are an excellent model to investigate the mechanisms 
linking neuronal position to circuit function. In mouse and zebrafish, FBM (nVII) neurons undergo tangential migration from rhombomere $4(\mathrm{r} 4)$ into r6 (mouse) and r6/7(zebrafish), with their axons exiting the hindbrain from r4 (Chandrasekhar, 2004; Chandrasekhar et al., 1997; Guthrie, 2007). The trigeminal $(\mathrm{nV})$ motor axons (whose cell bodies are located in $\mathrm{r} 2$ and $\mathrm{r} 3$ ) and FBM motor axons innervate the jaw and gill muscles in zebrafish, and jaw muscles, facial muscles and tongue in mammals (Chandrasekhar, 2004; Higashijima et al., 2000; Lumsden and Krumlauf, 1996; Song, 2007).

Given that FBM neuron migration is evolutionarily conserved, potential mechanisms linking aberrant neuronal position and defective circuit function have been examined in FBM migration mutants in mice (Thoby-Brisson et al., 2012) and zebrafish (McArthur and Fetcho, 2017). In Looptail $(L p)$ mutant mice, defective in function of the Wnt/Planar Cell Polarity (PCP) gene Vang/2, FBM neurons fail to migrate caudally out of $r 4$ (Glasco et al., 2012). Since the embryonic parafacial nucleus (e-pF), a neuronal population that controls breathing, develops adjacent to the FBM neurons, mispositioning of the FBM neurons in $L p$ mutants could impact epF development and function. Even though e-pF neurons showed a broad aberrant distribution in $L p$ mutants, they developed characteristic network properties, and established functional connections with other brainstem oscillators regulating breathing (Thoby-Brisson et al., 2012). This work suggests that functional outputs of neuronal circuits critical for survival 
(such as breathing) are unlikely to be severely impacted by defective neuronal positioning.

In a similar study done with zebrafish, McArthur and Fetcho (2017) investigated the developmental and functional consequences of failed FBM neuron migration using mutants defective in Wnt/PCP components (Gray et al., 2011). In landlocked (Ilk) and prickle1b (pk1b) mutants, carrying loss of function mutations in Scribble1a and Prickle1b, respectively, FBM neurons failed to migrate out of $r 4$, yet appear to innervate jaw muscles normally (Mapp et al., 2010; McArthur and Fetcho, 2017; Wada et al., 2005). Importantly, although FBM cell bodies and dendrites were mispositioned in the mutants, the organization of neurons within the motor nucleus according to age and target muscle was not affected. Remarkably, electrophysiological recording and $\mathrm{Ca}^{2+}$ imaging of individual FBM neurons and groups of FBM neurons, respectively, showed that activity patterns were similar between mutants and their wildtype siblings. Moreover, opercular movement, generated by FBM-innervated muscles, was essentially normal in mutants. This study again suggests that the functional output of neuronal networks regulating critical functions is resilient to changes in neuronal position and highlights the robustness of neuronal networks meditating essential physiological functions. We have tested this idea of resiliency again in our studies and report that mispositioning of FBM neurons can have subtle but profound effects on the branchiomotor circuit and its output. 
To examine the functional consequences of defective FBM migration, we used the zebrafish mutant off-limits (olt), which inactivates frizzled3a (fz3a), a Wnt/PCP gene (Wada et al., 2006). In olt mutants, FBM neurons fail to migrate caudally out of $\mathrm{r} 4$. Genetic mosaic analysis showed that $f z 3 a$ functions in a non-cell autonomous manner during FBM migration; consistent with this, $f z 3 a$ is expressed broadly in the hindbrain and surrounding tissues during the migratory period (Wada et al., 2006). Interestingly, olt mutants look morphologically normal and the homozygous mutant larvae can grow into viable and fertile adults (Wada et al., 2006), suggesting that the consequences of defective FBM positioning on survival are minimal. Nevertheless, it is important to evaluate the effects of FBM mispositioning on branchiomotor circuit organization, assembly, and output.

We evaluated the functional output of the FBM neurons using a jaw movement assay developed in our lab. Off-limits mutants exhibited reduced gape (opening and closing the mouth) frequency compared to their wildtype siblings. This decrease in gape frequency correlated with reduced food intake; i.e., olt mutants ate poorly. While patterns of innervation and neuromuscular junction (NMJ) structure were largely similar between olt mutants and wildtype siblings, there were subtle defects in axon fasciculation and neuronal activity. These studies establish the foundation for further investigating the role of neuronal position on circuit function. 


\subsection{RESULTS}

\subsubsection{Ontogeny of Lower Jaw Movement In Wildtype}

\section{Zebrafish Larvae}

The cranial motor neurons located in the vertebrate hindbrain, called branchiomotor neurons, innervate muscles of the jaw, face, and neck (Chandrasekhar, 2004; Guthrie, 2007). In zebrafish, they innervate muscles of the lower jaw and gills. To investigate the consequences of defective positioning of facial branchiomotor (FBM) neurons on circuit function and behavioral output, we developed a quantitative assay to evaluate jaw movement in zebrafish larvae. The trunks of 3-9 days post fertilization (dpf) larvae were mounted in agarose in a lateral orientation with the head free to move (Fig. 4.1 A). The spontaneous opening of the mouth due to lower jaw movement, termed gape, was recorded by timelapse imaging and analyzed using custom gape detection software (Kassim et al., 2018) (Fig. 4.1; Media 1, 2, 3). In 3 dpf Tg(is/1:GFP) (Higashijima et al., 2000) larvae, there was no jaw movement. Gape frequency, the rate of mouth opening, increased sharply between 3-5 dpf, increased further by $7 \mathrm{dpf}$, and maintained a stable high level up to $9 \mathrm{dpf}$ (Fig. 4.1 B). Gape magnitude, a measure of the mouth opening, also increased sharply between 3-5 dpf and stabilized thereafter (Fig. 4.1 C). Interestingly, the gape frequency in 5-9 dpf larvae exhibited a broad range 
due to the presence of two distinct jaw movement patterns: a distributed pattern with gape events uniformly distributed throughout the observation period, and a clustered pattern with tightly clustered events $(<10)$ with intervals of inactivity (Fig. 4.1 D). The proportions of the two patterns were similar between different ages, with the distributed pattern being the predominant one (Fig. 4.1 E). The ratio of eye diameter to head height at the jaw level did not change significantly between 3-9 dpf (Fig. 4.1.2; data not shown), indicating that the changes in gape magnitude are not due to differential growth of the jaw with age. These data demonstrate that jaw movement is a stable and developmentally reproducible behavioral output of the branchiomotor neuron circuits.

\subsubsection{Changes in Branching And Pre-Synaptic}

\section{Structures of Branchiomotor Axons May Drive}

\section{Increases in Jaw Motor Output}

The developmentally regulated changes in jaw gape frequency and gape magnitude may reflect the progression of specific events associated with the organization of the branchiomotor circuits and their innervation of the jaw muscles. While trigeminal branchiomotor $(\mathrm{nV})$ and FBM $(\mathrm{nVII})$ axons have fully extended over the mandibular and hyoid muscles by $3 \mathrm{dpf}$ (Higashijima et al., 2000), long before overt jaw movement, further expansion of outgrowth patterns after $3 \mathrm{dpf}$, and pre-and post-synaptic 
changes at the neuromuscular junctions (NMJs) between 3-9 dpf have not been studied.

We examined whether branchiomotor axon outgrowth patterns ramified further between 3-9 dpf, when jaw movements become fully established. We imaged these structures in live, embedded $T g$ ( $\alpha$ actin:GFP); $\operatorname{Tg}(z C R E S T 1: m R F P)$ larvae containing GFP-expressing muscles (Higashijima et al., 1997) and mRFP-expressing branchiomotor axons (Mapp et al., 2010) (see Materials and Methods; Media 6). While the branchiomotor axon fascicles extending on the intermandibularis posterior (imp), interhyoideus (ih), and hyohyoideus (hh) muscles were largely similar in 3-9 dpf larvae (Fig. 4. 2 A, D, G, J), there was a marked increase in branching of the fascicles between 3-5 dpf, coinciding with the increase in gape frequency and magnitude. A prominent FBM axon branch innervating the superficial membranous muscles (Higashijima et al., 2000) also emerged between 3-5 dpf (Fig. 4.2 A, D).

While FBM axon branching increases between 3-5 dpf, the increase in gape frequency and gape magnitude during this period could also result from changes at the NMJs on the imp, hh, and ih muscles. Therefore, we examined presynaptic structures at the jaw muscles by staining $T g$ (is/1:GFP); $\operatorname{Tg}$ ( $\alpha$-actin:GFP) larvae with an antibody against Synaptic Vesicle protein 2a (SV2a Ab) (Portela-Gomes et al., 2000), and quantified their volumes using Leica LAS X software (see Methods; Fig. 4.1.3). There was a significant increase in presynaptic volumes on 
ima/imp, hh and ih muscles from 3-7 dpf (Fig. 4.2 B, E, H, M), concomitant with the increases in gape frequency and magnitude (Fig. 4.1 B, C). Interestingly, there was significant decrease in presynaptic volumes on these muscles from 7-9 dpf (Fig. 4.2 H, K, M), corresponding to a decrease in gape parameters by $9 \mathrm{dpf}$ (Fig. 4.1 B, C).

Acetylcholine receptors $(\mathrm{AChR})$ in the postsynaptic structures at the jaw NMJs were stained in live larvae with alpha-bungarotoxin (aBTX) conjugated with Alexa Fluor 568 (Panzer et al., 2005). Although there was high variability, the volumes of $\mathrm{AChR}$ clusters on the imp, hh, and in muscles exhibited large increases from 3-7 dpf (Fig. 4.2 C, F, I, N) and a subsequent decrease by $9 \mathrm{dpf}$.

Taken together, these results suggest that increases in FBM axon branching on the jaw muscles and a significant increase in presynaptic volumes at the jaw NMJs can contribute to the onset of sustained jaw movement by $5 \mathrm{dpf}$.

\subsubsection{Jaw Movement is Defective in the off limits}

\section{(olt) Neuronal Migration Mutant}

Previous studies have shown that a reduction in FBM neuron number and mispositioning of FBM neurons can disrupt food intake and opercular muscle movement, respectively (Allen et al., 2017; McArthur and Fetcho, 2017). Therefore, we tested whether jaw movement was affected in offlimits (olt) mutants, which are deficient in frizzled3a ( $f z 3 a$ ) function, and 
where FBM neurons fail to migrate caudally out of $r 4$ (Fig. 4.3 A, B) (Wada et al., 2006). Jaw movement was imaged and analyzed in wildtype siblings and olt mutant larvae as described earlier. There was a significant decrease in gape frequency in 5, 7 and $9 \mathrm{dpf}$ olt mutants, with wildtype siblings exhibiting gape frequencies roughly double that of olt mutants at every age (Fig. 4.3 C; Media 4, 5). While gape frequencies in olt larvae were significantly lower than wildtype at all ages, the sharp increase in gape frequency between 3-5 dpf, and the plateauing of the frequencies between 7-9 dpf occurred normally in mutants (Fig. 4.3 C). The effects on gape magnitude were mixed, with significant reduction in olt mutants at 5 and $9 \mathrm{dpf}$, but not at $7 \mathrm{dpf}$ (Fig. $4.3 \mathrm{D}$ ). Furthermore, the proportions of "distributed" and "clustered" patterns of gape events were similar between wildtype and olt mutant larvae at all ages, although the "distributed" pattern was overrepresented in 7 and 9 dpf wildtype larvae (Fig. $4.3 \mathrm{E}$ ). These results suggest that while the proper migration and positioning of FBM neurons are important factors in generating jaw movement at a normal rate, they appear to play a minor or no role in the developmental onset of jaw movement or its magnitude, especially at $7 \mathrm{dpf}$ when larvae are actively foraging.

\title{
4.3.4 Defective Jaw Movements in olt Mutants are
}

\author{
Associated With Reduced Food Intake
}


Given the reduced jaw movement in olt mutant larvae, we wondered whether their ability to track and capture food particles (food intake) was also reduced. We employed a robust and sensitive feeding assay developed in our lab (Allen et al., 2017) to visualize fluorescent microsphere-coated food particles within the gut of immobilized larvae. Actively swimming $7 \mathrm{dpf}$ larvae were fed fluorescent food for 3 hours and assigned a feeding score (FS) from $0-3$, representing the distribution of the fluorescent microspheres within the gut, ranging from no fluorescence $(F S=0)$ to a fully fluorescent gut (FS=3) (Fig. 4.4 A; see Methods). Food intake in the olt mutant population was significantly reduced compared to wildtype (Fig. 4.4 B). While some olt mutant larvae ate just as well as most wildtype larvae, the distribution was greatly skewed toward low food intake ( $F S=0$ and 1 ) in mutants compared to wildtype siblings.

Given the broad expression pattern of $f z d 3 a$ (Wada et al., 2006), poor food intake in olt mutants may be a non-specific effect resulting from deformation of the jaw or an inability to swim normally. Alcian blue staining of jaw cartilage elements in $7 \mathrm{dpf}$ olt mutant larvae and wildtype siblings revealed no differences in the lengths of various cartilage elements or in the angles subtended by the elements between the two populations (Fig. 4.4 C-F), indicating that the lower jaw developed and was patterned normally in mutants. Swimming activity of $6 \mathrm{dpf}$ larvae imaged and analyzed with the DanioVision and EthoVision locomotion tracking software (Noldus; see Methods) revealed no differences in swimming 
distance or swimming duration between olt mutants and wildtype siblings (Fig. $4.4 \mathrm{G}, \mathrm{H}$ ). Taken together, these data suggest strongly that the reduced food intake seen in olt mutants results specifically from the reduced jaw movement of mutant larvae.

\subsubsection{FBM Axon Outgrowth to Jaw Muscles Occurs}

\section{Normally in olt Mutants}

With non-specific bases for reduced jaw movement in olt mutants ruled out, we tested whether specific aspects of the FBM axon pathway innervating the jaw muscles may be affected in mutant larvae. Although mutant FBM neurons appear to extend motor axons normally (Wada et al., 2006), their precise outgrowth and NMJs at the jaw muscles were not examined. Therefore, we 3D-reconstructed and quantified FBM axon outgrowth and branching in live $5 \mathrm{dpf} T g(z G R E S T: G F P)$ olt mutants using Leica LAS X software (see Methods; Fig. 4.1.4 ; Media 6). Since the migration defect is restricted to FBM neurons, trigeminal motor (nV) axon outgrowth was used as a control because these neurons are positioned normally in mutants (Fig. 4.5 A, B). The total lengths (of the primary fascicle and branches) for trigeminal motor and FBM axons from their hindbrain exit points up to the jaw muscles were unaffected in olt mutants and wildtype siblings (Fig. 4.5 C, D). Additionally, there were no significant differences between olt mutants and wildtype siblings in the number of branches arising from trigeminal motor and FBM axons in olt mutants and 
wildtype siblings (Fig. 4.5 C, D). Interestingly, while FBM axons exhibited more branching, and hence had higher total length, than trigeminal motor axons, there were no differences between olt mutants and wildtype siblings. These results demonstrate that FBM axon outgrowth and extension on jaw muscles occur normally in olt mutants and cannot contribute to defective jaw movements in mutant larvae.

\subsubsection{FBM Axons Extending on The Ih Muscle Are Frequently Defasciculated in olt Mutants}

While FBM axons extended normally to the jaw muscles in olt mutants, we could not rule out subtle axonal defects at the muscles where the NMJs develop. Therefore, we used $\operatorname{Tg}(z C R E S T: m R F P) ; \operatorname{Tg}(a-a c t i n: G F P)$ fish to examine axonal outgrowth in 3D on the jaw muscles in 5 dpf larvae using Leica LAS X software. Axon branching on specific jaw muscles was isolated in 3D and viewed using a reference mask (see Methods; Fig. 4.1.4; Media 6). We observed that the axons, especially on the in muscle, were aggregated into thicker fascicles in wildtype, whereas the mutant axons were often split into thinner fascicles (Fig. 4.5 E, F). Importantly, defasciculated axons, not situated on the muscle, were found at a significantly higher rate for olt mutant FBM axons extending on the in muscle $(\sim 80 \%)$ than for wildtype FBM axons ( 40\%) (Fig. 4.5 E, F, G). 
Although this defasciculation phenotype is subtle, it has the potential to impact in muscle activity, and could contribute to jaw movement defects in olt mutants.

\subsubsection{NMJs on Jaw Muscles Appear to be}

\section{Unaffected in olt Mutants}

Since FBM axon defasciculation defects are evident at the in muscle in olt mutants, it is possible that jaw NMJs are also affected in mutants.

Furthermore, given that increased presynaptic volume may drive the developmentally regulated increase in gape frequency (Fig. 4.2), reduced gape frequencies in olt mutants may result from smaller presynaptic structures. Therefore, we quantified presynaptic volumes (SV2a antibody staining) and postsynaptic acetylcholine clusters (aBTX labeling) in $7 \mathrm{dpf}$ olt mutants as described earlier (Fig. 4.2). The volumes of presynaptic structures on various jaw muscles were similar between olt mutants and wildtype siblings (Fig. 4.6 A, B). While there was a slight but consistent reduction in presynaptic volumes on the ih and hh muscles in mutants, these changes were not statistically significant (Fig. 4.6 E). Volumes of postsynaptic AChR clusters were largely unaffected on all muscle groups between $7 \mathrm{dpf}$ olt mutants and wildtype siblings, and were essentially identical for the ih and hh muscles (Fig. 4.6 C, D, F). These results indicate that the reduced jaw movement of olt mutants does not result 
from deficits in NMJ structures formed by branchiomotor axons on jaw muscles.

\subsubsection{FBM Neurons Are Less Active in Olt Mutants}

Since extensive characterization of FBM axon projections to and NMJs on jaw muscles revealed only subtle structural defects in olt mutants (Fig.

4.5), we wondered whether FBM neuronal activity might itself be affected in mutants. To test this possibility, we introduced the olt mutant allele into the $\operatorname{Tg}(z C R E S T 1: G C a M P 6 s)$ background where branchiomotor neurons express the calcium indicator GCaMP6s (see Methods). Wildtype and olt mutant larvae at $7 \mathrm{dpf}$ were anesthetized by aBTX injection and FBM neuron clusters were identified in r6 (normal migration) and $\mathrm{r} 4$ (defective migration), respectively. The neurons were imaged by time-lapse confocal microscopy (Fig. 4.7 A, B) and their $\mathrm{Ca}^{2+}$ activities were analyzed using Fiji (see Methods). Periodic calcium spikes were seen throughout the observation period in both wildtype and mutant larvae; however, the spikes occurred less frequently in mutants (Fig. 4.7 D). The frequency of $\mathrm{Ca}^{2+}$ spike events was significantly lower in olt mutants compared to their wildtype siblings (Fig. 4.7 C). Importantly, the frequency of spike events for trigeminal motor $(\mathrm{nV})$ neurons located in $\mathrm{r} 2$ was similar between wildtype and olt mutants, indicating that the reduction for FBM neurons is not a pleiotropic effect of the mutation. These results suggest reduced 
activity of FBM neurons may contribute to the jaw movement defect (lower gape frequency) of olt mutants.

\subsection{DISCUSSION}

Precise migration of newborn neurons is an integral part of the development of the nervous system. Disruption of neuronal migration can lead to brain abnormalities associated with neurodevelopmental disorders, which are characterized by behavioral deficits. However, the underlying mechanisms by which abnormal neuronal migration and positioning generate defects in circuit organization and function are poorly understood. Here, we employed the migration of facial branchiomotor (FBM) neurons as a model to investigate the consequences of defective neuronal migration on the organization and functional outputs of the motor circuit. In this report, we use the word "circuit" to specifically refer to the connection between the branchiomotor neurons and their jaw muscle targets. While visual, olfactory, gustatory and hypothalamic inputs to the FBM and other branchiomotor neurons are essential elements of the branchiomotor circuit controlling jaw movement, the roles of these neural components have not been addressed here. Our analysis of the FBM migration mutant off-limits (olt) revealed subtle structural and functional deficits in the circuits controlling jaw movement. Our work indicates that while neuronal circuit function is largely resilient to changes in positioning 
of component neurons, there is potential for disrupting functional (behavioral) outputs that impact health and survival.

\subsubsection{Developmental Changes in Jaw Movement Behavior May be Pre-Synaptically Driven}

Between 3-5 dpf, the frequency of jaw movement (gape) and its magnitude increased sharply from a very low level and remained elevated through $9 \mathrm{dpf}$. This time course of gape acquisition coincides with the time when zebrafish larvae start actively foraging for food (Budick and O'Malley, 2000; Carrillo and McHenry, 2016). While the motor output of the branchiomotor circuitry is established and becomes robust between 5$7 \mathrm{dpf}$, the branchiomotor axons have already extended on to the jaw muscles (Higashijima et al., 2000) and assembled neuromuscular junctions (NMJs) by $3 \mathrm{dpf}$. The lag between NMJ assembly and the onset of jaw movement may reflect the occurrence of additional events needed for the maturation of the circuit. These events could include changes in or further elaboration of the NMJs, arrival of inputs to the branchiomotor neurons from other brain regions, and acquisition of specific electrical properties by the motor neurons as a part of their differentiation process.

Our data strongly support a role for changes in NMJs between branchiomotor axons and jaw muscles (Fig. 4.2), but other drivers of their maturation cannot be ruled out. For example, the spontaneous and coordinated activities of zebrafish tectal neurons between 2-4 dpf, when 
the visual system is becoming functional (Pietri et al., 2017), are driven in part by changes in their dendritic arborization and neurotransmitter identity (Boulanger-Weill et al., 2017). In another example of circuit maturation, the onset of robust activity in the respiratory circuits controlling breathing in mice between embryonic day 16 (E16) and E18 is driven by acquisition of neuromodulatory inputs and inputs from other rhythmic centers (Viemari et al., 2003). While the roles of neuromodulation and other inputs in circuit maturation can be readily studied by pharmacological manipulation in in vitro preparations, these approaches are not feasible for examining branchiomotor circuit maturation driving jaw movement in zebrafish. However, imaging of neuronal activity and trans-synaptic labeling to identify inputs from other brain regions can be employed to identify potential maturation mechanisms in addition to NMJ changes that we have identified.

Our data suggest that the developmental onset of jaw movements between 3-5 dpf is a prerequisite for larvae to forage and capture food from $5 \mathrm{dpf}$. These jaw movements could potentially represent buccal movements, which also increase between 3-9 dpf (Jonz and Nurse, 2005), however a comparison of ventilation frequencies (generated by buccal movement) in 3-9 dpf larvae under normoxic and hypoxic conditions to 3-9 dpf gape frequencies reveals conflicts. First, the ventilation frequency is $\sim 0$ in $6 \mathrm{dpf}$ larvae under normoxia, whereas gape frequency at $5 \mathrm{dpf}$ (mean $\sim 0.8 \mathrm{~Hz}$ ) is closer to the ventilation frequency under hypoxia. On 
the other hand, gape frequency increases $\sim 33 \%$ from $5-7 \mathrm{dpf}$, whereas ventilation frequency under hypoxia increases $\sim 250 \%$ from 6-7 dpf (Jonz and Nurse, 2005). This suggests that developmental changes in gape frequency do not correspond to changes in ventilation frequencies either under normoxia or hypoxia. Hence, while larvae in our experimental conditions may experience hypoxia, the developmental maturation of gape follows a different trajectory than maturation of ventilation - the latter appears to result from the innervation of oxygen-sensing neuroepithelial cells in the gill filaments (Jonz and Nurse, 2005), whereas the former could result from pre-synaptic changes at the jaw NMJs (Fig. 4.2).

\subsubsection{Defective FBM Neuron Position in olt Mutants is Associated With a Significant Functional Deficit in The Motor Circuit}

We have documented two defective behavioral phenotypes in olt mutants that represent functional outputs of the branchiomotor circuit. First, gape (jaw opening) frequency is significantly reduced in mutants. Second, olt mutants eat less than their wildtype siblings. These data are the first demonstration of a defective functional output associated with abnormal positioning of FBM neurons.

Breathing rhythms in mouse embryos, a motor output driven in part by the e-pF neurons that are immediately adjacent to the FBM neurons 
(Thoby-Brisson et al., 2009), are not disrupted in Looptail mutants where FBM neurons fail to migrate out of $r 4$ (Thoby-Brisson et al., 2012). Even though e-pF neurons are significantly displaced in Looptail mutants due to the mispositioning of FBM neurons, the output of the respiratory motor circuit was largely unaffected, suggesting that functional outputs of circuits that are critical for survival may be resistant to changes in neuronal position (Thoby-Brisson et al., 2012). In contrast, our data suggest for the first time that larval jaw movement, a motor output that is necessary for food intake and survival, can be modified by changes in FBM neuron position. Interestingly, while the lower frequency of jaw movements is correlated with reduced food intake in olt mutants, it is not clear whether this feeding defect impacts survival since homozygous mutant olt larvae can grow into viable and fertile adults in a favorable lab environment with plentiful food and lack of competition from wildtype siblings. Further analysis is needed to evaluate whether food intake deficits measured in our assay translate to reduced survival in challenging environments that incorporate competition for limited food.

McArthur and Fetcho (2017) performed extensive analysis of landlocked (Ilk) and prickle1b mutants, both of which exhibit similar FBM neuron migration defects as olt mutants. Their studies did not reveal any changes in branchiomotor neuronal organization or branchiomotor circuit output, indicating that this motor circuit is resilient to changes in neuronal position, perhaps through compensatory mechanisms (McArthur and 
Fetcho, 2017). Furthermore, while this study analyzed and found no effect on opercular movement, a behavior driven by an FBM neuron-innervated muscle, one cannot rule out deficits in jaw muscle innervation and movement in Ilk mutants, as seen in olt mutants. Indeed, preliminary analyses indicate that gape frequency and food intake are reduced in $/ / k$ mutants (Asante and Chandrasekhar, unpublished data). Detailed analysis is needed to determine whether the behavioral deficits in Ilk mutants are distinct and different from those in olt mutants, and whether these are accompanied by changes in branchiomotor axon morphologies and NMJ structures.

Our work is the first demonstration of motor circuit output defects resulting from aberrant neuronal position, where a direct link between neuronal mispositioning and circuit deficits can be readily established. However, there are several examples of human neuronal migration disorders (NMDs) and mouse NMD models where mispositioning of neurons is associated with behavioral deficits (Moffat et al., 2015). Aberrant migration of pyramidal projection neurons, or cortical interneurons and hippocampal neurons are associated with a broad range of cognitive and motor deficits seen in autism and schizophrenia, respectively (Moffat et al., 2015; Muraki and Tanigaki, 2015; Pan et al., 2019). Given the complex etiology of these disorders, it remains challenging to establish direct mechanistic links between mispositioned cortical or hippocampal neurons and specific behavioral deficits. Because 
of the relatively simple organization of the branchiomotor circuit mediating jaw movements, our ongoing studies will facilitate the elucidation of the molecular and cellular consequences of defective neuronal migration (and positioning) on circuit output.

\subsubsection{Are Branchiomotor Axon and Activity Defects in olt Mutants a Consequence of FBM Neuron}

\section{Mispositioning?}

Our data indicate that there are subtle defects in branchiomotor axon fasciculation and FBM neuron activity (GCaMP6 fluorescence) in 7 dpf olt mutants (Figures 4.6 and 4.7) concomitant with reduced jaw movements. While we interpret these defects to be the consequence of FBM neurons being mislocated in $\mathrm{r} 4$ due to their failure to migrate caudally, we cannot rule out that these defects arise independently of FBM neuron mispositioning. Since $f z 3 a$, the PCP gene inactivated in olt mutants, is broadly expressed in neural and non-neural tissues (Wada et al., 2006) and loss of $F z 3$ has been shown to disrupt the growth of several axon tracts in the developing mouse brain (Hua et al., 2014; Wang, 2006; Wang et al., 2002), FBM axon defects might be expected in olt mutants. Interestingly, most of the defasciculation defects in olt mutants are found on the ih and hh muscles that are innervated by FBM axons, suggesting the defasciculation phenotype may be preferentially associated with axons 
extended by the mispositioned motor neurons. Moreover, other aspects of axon outgrowth like length and branching from FBM and trigeminal axons are unaffected indicating that there are no widespread defects in neuronal development in olt/fz3a mutants, and consistent with the normal development of several axon pathways in Fz3 mutant mice (Hua et al., 2014). The reduced frequency of $\mathrm{Ca}^{2+}$ transients in olt mutants could be the consequence of the mispositioned FBM neurons expressing an altered repertoire of ion channels. Alternatively, loss of $f z 3 a$ may lead to dysregulation of the non-canonical Wnt/Calcium pathway (Slusarski and Pelegri, 2007), resulting in altered $\mathrm{Ca}^{2+}$ dynamics within FBM neurons. Cell autonomous and non-autonomous effects of $f z 3 a$ loss on FBM neurons can be addressed by expression profiling analysis.

The concomitant decreases in jaw movement (gape frequency) and $\mathrm{Ca}^{2+}$ spike activity in olt mutants may suggest a direct link between putative firing of FBM neurons and contraction of the jaw muscles they innervate. Indeed, the firing patterns of FBM neurons and opercular movements overlap in frequency (McArthur and Fetcho, 2017). However, the observed $\mathrm{Ca}^{2+}$ spike frequency $(\sim 0.02 \mathrm{~Hz}$; Fig. 4.7$)$ is roughly 50 -fold lower than the gape frequency ( 1 Hz; Fig. 4.1) at $7 \mathrm{dpf}$. This could be a technical issue, since we used GCaMP6s to record $\mathrm{Ca}^{2}+$ spikes, rather than GCaMP6f (McArthur and Fetcho, 2017), resulting in a failure to detect rapid spikes associated with FBM neuron firing. Nevertheless, our data have established a link between activity changes within FBM neurons 
and the jaw movement output of the branchiomotor circuit. The modulation of this link by changes at the jaw NMJs and by regulatory inputs to the FBM neurons from other brain regions needs further study.

In conclusion, our analysis of jaw movement defects in olt mutants suggests strongly that a functional output of the branchiomotor circuit is impacted by the mispositioning of one of the motor neuron populations driving the behavior. We also document changes in circuit structure and activity that could underlie the alteration in the motor output. In the future, we will further address the link between neuronal mispositioning and motor output by employing a variety of conditions to systematically vary FBM neuron position within the zebrafish hindbrain and evaluating their impacts on jaw movement and food intake, behavioral outputs of the branchiomotor circuit that are essential for survival.

\subsection{MATERIALS AND METHODS}

\subsubsection{Animals}

Adult zebrafish (Danio rerio) were maintained according to standard protocols (Westerfield, 2000) approved by the Animal Care and Use Committee at the University of Missouri. Embryos were obtained through natural mating and were incubated in E3 medium at $28.5^{\circ} \mathrm{C}$. Embryos were staged by counting somites at $16-18 \mathrm{hpf}$ (hours post fertilization) 
(Kimmel et al., 1995), and pigmentation was prevented by $0.003 \%$ phenylthiourea treatment from $18 \mathrm{hpf}$.

The $T g(i s / 1: G F P)$ line (Higashijima et al., 2000) expresses GFP in all branchiomotor neurons (nV, nVII, nIX and nX) throughout development, and was used to characterize the developmental acquisition of jaw movement. $\operatorname{Tg}(z C R E S T 1: m R F P)$ expresses membrane RFP in branchiomotor neuron cell bodies and axons (Mapp et al., 2010). $\operatorname{Tg}(\alpha-$ actin:GFP) expresses GFP in most muscles, including the jaw, gill and ocular muscles (Higashijima et al., 1997). Tg(zCREST1:GCaMP6s) was generated by Tol2 transgenesis. The Tol2 destination vector was generated by Gateway Cloning of p5E-zCREST1, pENTR-GCaMP6s, and p3E-polyA constructs. This line expresses GCaMP6s in branchiomotor neurons at low levels until $48 \mathrm{hpf}$, at a much higher level between 4-7 dpf (days post fertilization). The off-limits (o/t ${ }^{\text {w6899}}$ ) mutant allele contains a loss-of-function missense mutation in the frizzled3a ( $f z 3 a$ ) gene (Wada et al., 2006).

\subsubsection{Jaw Movement Assay}

After embryos hatched at $2 \mathrm{dpf}$, they were screened based on facial branchiomotor (FBM) neuron migration. Larvae at $2 \mathrm{dpf}$ were placed in $28.5^{\circ} \mathrm{C}$ 14-hour light and 10-hour dark cycles (LD 14:10) incubator. Larvae at $3,5,7$ or $9 \mathrm{dpf}$ were placed into a multi-lane $1.2 \%$ agarose mold petri dish minimizing liquid transfer. Low melting agarose (2\%) in E3 was 
applied gently over the larvae, which were oriented laterally into the agarose troughs. 1.2\% agarose in E3 (Agarose LE (Gold Biotechnology)) was applied over the lower trunk region to trap the larvae. Excess agarose was removed from the head region, allowing for full movement of the lower jaw (Media 1-5). Each larva was recorded under brightfield with an Olympus SZX12 stereomicroscope (90X magnification) fitted with a Retiga 2000 camera (QImaging, Inc.) for one minute after jaw movement was observed (18 frames per second, 600 frames) and removed after 10 minutes if no jaw movement occurred. For experiments involving olt mutants, the person imaging the larvae was blinded to the larvae's genotypes, ensuring that each movie was analyzed in an unbiased manner using proprietary software (Fig. 4.1.1) (Kassim et al., 2018).

\subsubsection{Food Intake (Feeding) Assay}

Our procedure is extensively described in (Allen et al., 2017). Briefly, 1015 larvae were placed in $5 \mathrm{ml}$ of E3 medium containing $2 \mathrm{mg}$ Larval fish food (Zeigler $<100$ microns) coated in yellow fluorescent microsphere (Molecular Probes/ThermoFisher) mixture for 3 hours. At the end of the feeding period, larvae were anesthetized with $0.02 \%$ tricaine (Western Chemical Inc.), mounted laterally, and scored based on the amount of fluorescent food in the gut. Larvae were examined with an Olympus SZX12 stereomicroscope equipped with epifluorescence optics at 40-90X fitted with a Retiga 2000 camera (QImaging, Inc.) and assigned a score 
ranging from $0-3$. A feeding score of ' 0 ' corresponded to the larvae with no fluorescent signal within the gut, while a score of '3' corresponded to fluorescence throughout the gut (Fig. 4.4 A). The individual scoring each larva was blinded to the treatment and genotype.

\subsubsection{Swimming Assay}

At $2 \mathrm{dpf}$, mutant larvae and wildtype siblings were separated based on FBM migration phenotype and incubated at $28.5^{\circ} \mathrm{C}$. At $6 \mathrm{dpf}$, larvae were placed in individual wells of a 24-well plate and observed at room temperature $\left(21-22^{\circ} \mathrm{C}\right)$. The DanioVision system and EthoVision XT 8.0 locomotion tracking software (Noldus) were used to measure the distance moved and moving duration. Larvae were allowed to acclimate to the dark for 30 minutes, and distance moved and moving duration parameters were analyzed (Fig. 4.4 G-H).

\subsubsection{Alcian Blue Staining}

The alcian blue staining method (Nissen et al., 2006) was adapted to visualize the cartilage structure in the larval head. Larvae were fixed in $4 \%$ paraformaldehyde (PFA) overnight at $4^{\circ} \mathrm{C}$, washed twice with PBST (PBS containing $0.1 \%$ Tween 20 ) and dehydrated through $50 \%$ methanol $(\mathrm{MeOH})$ in PBST into $100 \% \mathrm{MeOH}$. This was followed by overnight staining in $0.15 \%$ alcian blue (Sigma) in ethanol $(\mathrm{EtOH})$ solution at room temperature with gentle agitation. Larvae were washed with $100 \% \mathrm{EtOH}$, 
rehydrated through 50\% EtOH/PBST into 100\% PBS and digested in $0.05 \%$ trypsin (in 1XPBS) for 24 hours, and rinsed in PBST. Larvae were bleached in $3 \% \mathrm{H}_{2} \mathrm{O}_{2} / 1 \% \mathrm{KOH}$ at room temperature, checking every 5 minutes until desired transparency was reached and transferred to $70 \%$ glycerol through a glycerol series. Larvae were imaged on a Leica DM widefield microscope. Jaw cartilages were analyzed for length and angles related to the head skeleton anatomy with ImageJ $(\mathrm{NIH})$.

\subsubsection{Immunostaining And Analysis of}

\section{Neuromuscular Junctions}

Our procedure was adapted from (Hunter et al., 2011). Zebrafish expressing GFP in the jaw muscles ( $T g(\alpha$-actin:GFP)) were used in the analysis. The pre-synaptic compartment of NMJs was stained using a monoclonal antibody against the pre-synaptic protein SV2a

(Developmental Studies Hybridoma Bank, 1:200 dilution) and a goat antimouse Alexa Flour 568 (Life Technologies, 1:500 dilution) secondary antibody. The jaw muscles were stained using rabbit anti-GFP (Invitrogen, 1:2000 dilution) primary antibody and chicken anti-rabbit Alexa Fluor 488 (Invitrogen; 1:500 dilution) secondary antibody.

Post-synaptic structures at NMJs were visualized using a protocol provided by Dr. Diane Sepich (Washington University School of Medicine, St. Louis), modified from a previous study (Panzer et al., 2005).

Transgenic larvae expressing GFP in the jaw muscles were used in these 
studies. Live larvae were incubated in Alexa Fluor 594-conjugated abungarotoxin ( $\mathrm{ABTX} ; 10 \mu \mathrm{g} / \mathrm{ml}$; Invitrogen) for $30 \mathrm{~min}$ in $\mathrm{E} 3$ medium containing $15 \%$ DMSO.

Larvae were embedded in 1\% LMP agarose (SV2) or anesthetized in $0.02 \%$ Tricaine $(\mathrm{aBTX})$ for imaging. Bidirectional Z-stack images $(1 \mu \mathrm{m}$ thickness, $140-200$ slices) were acquired with a Leica TCP SP8 MP inverted confocal microscope with a 20X/0.75 water immersion objective. Confocal stacks were processed in two channels to obtain 3D images with Leica Application Suite X (LAS X). 3D images from the GFP channel (468 $\mathrm{nm}$ ) (jaw muscles) were used as a mask overlay to examine the 3D images of the SV2-labeled presynaptic and a BTX-labeled postsynaptic structures in the 568/594 $\mathrm{nm}$ channel and analyzed using LAS X (Fig.

$$
\text { 4.1.3). }
$$

\subsubsection{Axon Imaging And Analysis}

Double transgenic zebrafish expressing membrane RFP $(\operatorname{Tg}(z C R E S T 1: m R F P))$ in the branchiomotor axons and GFP in the jaw muscles ( $T g(\alpha$-actin:GFP $))$ were used to analyze axon morphology. Larvae were anesthetized with $0.02 \%$ tricaine and bidirectional Z-stack images $(1 \mu \mathrm{m}$ thickness, $15-25$ slices) were collected with a Leica TCP SP8 MP confocal microscope with a 20X/0.75 water immersion objective. Leica Application Suite X (LAS X) software was used to generate 3D images from the stacks of confocal images from the RFP channel (axons) 
and GFP channel (jaw muscles). Axon length and branching number in the lateral view were traced in the 3D image by examining each slice. Images were analyzed by LAS X and Fiji (NIH) (Fig. 4.1.4; Media 6).

\subsubsection{Calcium Imaging And Analysis}

Transgenic larvae expressing the calcium indicator GCaMP6s in the branchiomotor neurons ( $\operatorname{Tg}(z C R E S T 1: G C a M P 6 s)$ generated in our lab) were used for this analysis. Larvae were maintained in an LD 14:10 incubator. One hour before imaging, larvae were injected with $4.6 \mathrm{ng}$ of $\alpha$ bungarotoxin (Alomone Labs) to paralyze and prevent twitching. Larvae were mounted dorsally on a coverglass-bottom petri dish in $0.2 \%$ agarose in E3 and covered with E3 after setting. Imaging was performed using a Leica TCS SP8 MP inverted confocal microscope with a 20X/0.75 water immersion objective. Larvae were imaged at 2 frames per second for 5 minutes (Media 7, 8). Images were analyzed using Fiji (NIH) and regionsof-interest (ROIs) were manually drawn corresponding to FBM neurons and trigeminal neurons based on GCaMP expression and location in the hindbrain. ROI for background subtraction was performed on every frame in the same time series as FBM neurons and trigeminal neurons ROI,

yielding the background-corrected trace. The $\mathrm{Ca}^{2+}$ spike events were counted from the traces and manually validated by two observers independently watching the time-lapse recordings. 


\subsection{Statistical Analysis}

For jaw movement analysis from 3-9 dpf (Fig. 4.1) Chi-square test and One-way analysis of variance (ANOVA) with post hoc Tukey's HSD (honest significance difference) were employed. For comparisons between olt mutants and wildtype siblings (various Figures), two-tailed student ttest or Chi-square test (Fig. 4.3) or Fisher's exact t-test (Fig. 4.5) was used. See Figure legends for details.

\subsection{ACKNOWLEDGEMENTS}

We thank members of the Chandrasekhar lab for discussion and fish care, and the D. Cornelison lab (University of Missouri) for discussion and support. We thank Dr. Diane Sepich (Washington University) for advice and protocols for labeling neuromuscular junctions and GCaMP imaging, and Dr. Lila Solnica-Krezel (Washington University) for the pENTR-GCaMP6s vector. We thank Dr. Alexander Jurkevic and Dr. Robert Baker in the University of Missouri Molecular Cytology Core Facility for extensive help and training with confocal microscopy and Leica LAS-X software. The $T g(i s / 1: G F P), T g(\alpha$-actin:GFP), and off-limits lines were provided by Dr. Hitoshi Okamoto (RIKEN, Japan), and the Tg(zCREST1:mRFP) line was provided by Dr. Victoria Prince (University of Chicago). EA was supported in part by an NIH IMSD grant R25GM056901 to Dr. Mark Hannink (University of Missouri). This work 
was partially supported by NIH grants R01NS110915 (KP), U.S. Army

Research Laboratory project W911NF-18-2-0285 (KP), and

R03HD098555 (AC), and bridge funds from the Bond Life Sciences

Center (AC). 


\subsection{FIGURES AND LEGENDS}

Figure 4.1: Ontogeny of lower jaw movement in wildtype zebrafish larvae
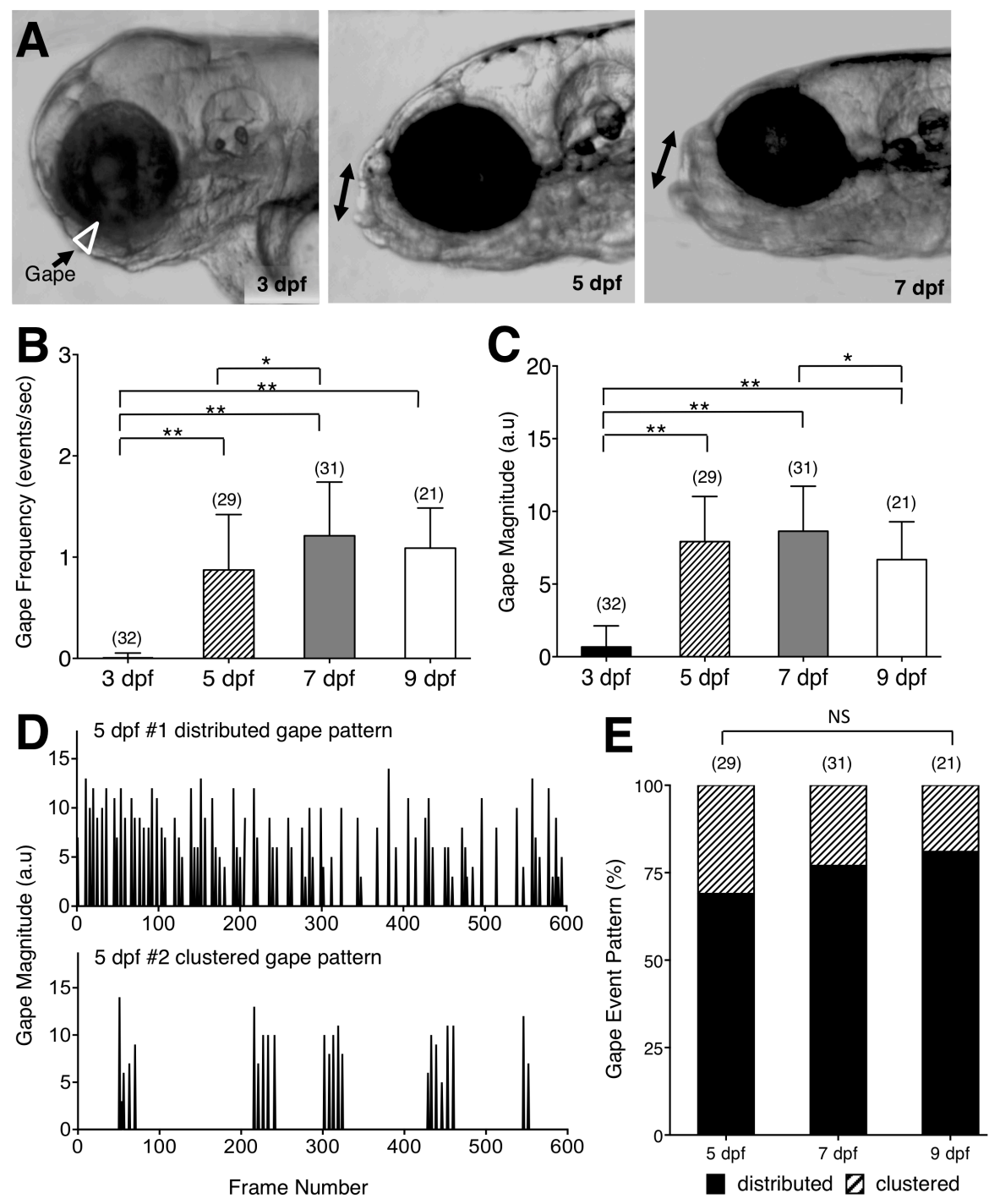
Figure 4.1: Ontogeny of lower jaw movement in wildtype zebrafish larvae

(A) $\mathrm{Tg}$ (is/1:GFP) larvae from 3, 5 and $7 \mathrm{dpf}$ were mounted in a lateral position under a brightfield microscope in agarose with the head free to move and the opening of the mouth due to lower jaw movement (gape; white triangle, double arrows) was recorded and analyzed using custom software. (B, C, E) Pooled data from 3 experiments (number of larvae in parenthesis). (B) Gape frequency increased sharply from 3 to $7 \mathrm{dpf}$, plateauing by 9 dpf. (C) Gape magnitude, a measure of the amount of jaw movement, increased sharply between 3 and $5 \mathrm{dpf}$ and plateaued thereafter. (D) There were two patterns of gape events - Distributed, with jaw movements distributed uniformly throughout the observation period, and Clustered, with tightly clustered jaw movements with intervals of inactivity. (E) The distributed gape pattern was predominant at every age examined, and there was no significant difference between 5,7 and $9 \mathrm{dpf}$. Statistical analysis was carried out with Chi-square test and One-way analysis of variance (ANOVA) with post hoc Tukey's HSD (honest significance difference). NS: not significant, ${ }^{*} p<0.05,{ }^{* *} p<0.01$. 
Figure 4.2: Developmental changes in branchiomotor axon branching and synaptic structures at the jaw neuromuscular junctions

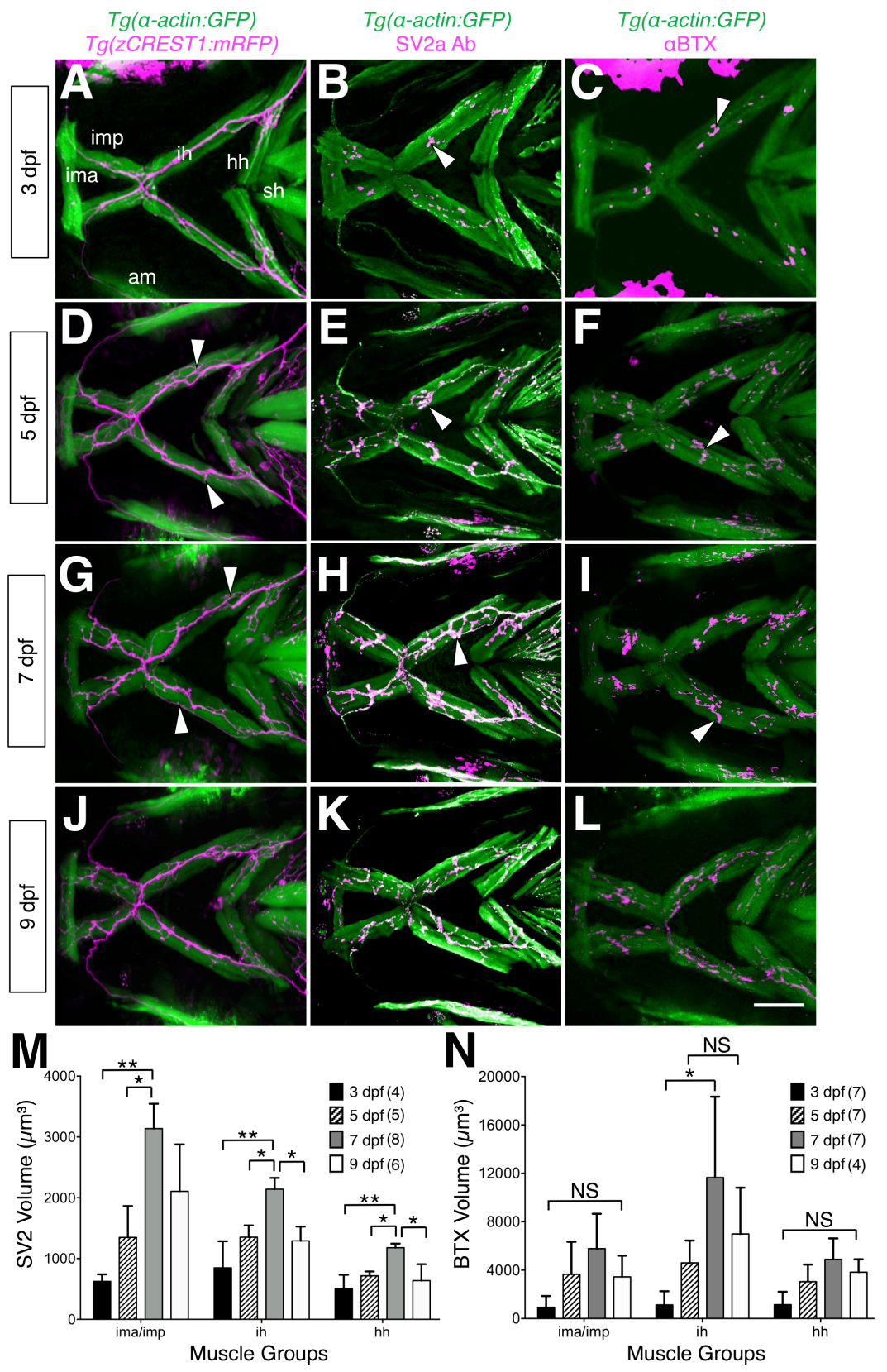


Figure 4.2: Developmental changes in branchiomotor axon branching and synaptic structures at the jaw neuromuscular junctions

Ventral views with anterior to the left of the jaw musculature. (A, D, G, J) Axon (magenta) outgrowth on jaw muscles (green) in 3, 5, 7, and $9 \mathrm{dpf} T g$ ( $\alpha$-actin:GFP); $\operatorname{Tg}(z C R E S T 1: m R F P)$ larvae. 3D rendering of muscles and axons were overlaid to determine axon position relative to the muscles. Fine axon branches (white arrowheads) across the jaw muscles increased as larvae aged. (B, E, H, K) SV2a antibody (magenta) labeled presynaptic structures on the jaw muscles (green) in 3, 5, 7, and $9 \mathrm{dpf}$ $\operatorname{Tg}($ is/1:GFP); $\operatorname{Tg}(\alpha$-actin:GFP) larvae. 3D rendering was used to visualize the presynaptic regions in contact with the muscles (arrowheads) and calculate their volumes. (M) Presynaptic volumes increased greatly from 3-7 dpf larvae, especially for ima/imp and ih muscles. There was a significant increase from 5-7 dpf in the ima/imp, in and hh muscles. Both ih and hh muscles had a significant decrease in presynaptic volume from 7 to $9 \mathrm{dpf}$ larvae. (C, F, I, L) Ventral view with anterior to the left. Acetylcholine receptor (AChR) clusters (arrowheads) were labeled with alpha-bungarotoxin (aBTX) (magenta) on the jaw muscles (green) in live 3, 5, 7, and $9 \mathrm{dpf} T g(\alpha$-actin:GFP) larvae. (N) Although AChR cluster volumes on all muscles tend to increase from 3-7 dpf, these changes were not significant. Data pooled from 3 to 5 experiments (number of larvae in parenthesis). Statistical analysis was carried out with Chi-square test and 
One-way analysis of variance (ANOVA) with post hoc Tukey's HSD (honest significance difference). NS: not significant; ${ }^{*} p<0.05,{ }^{* *} p<0.01$. Abbreviations: ima/imp, intermandibularis anterior/intermandibularis posterior; ih, interhyal; hh, hyohal; sh, sternohyoideus. 
Figure 4.3: olt mutants have reduced jaw movement
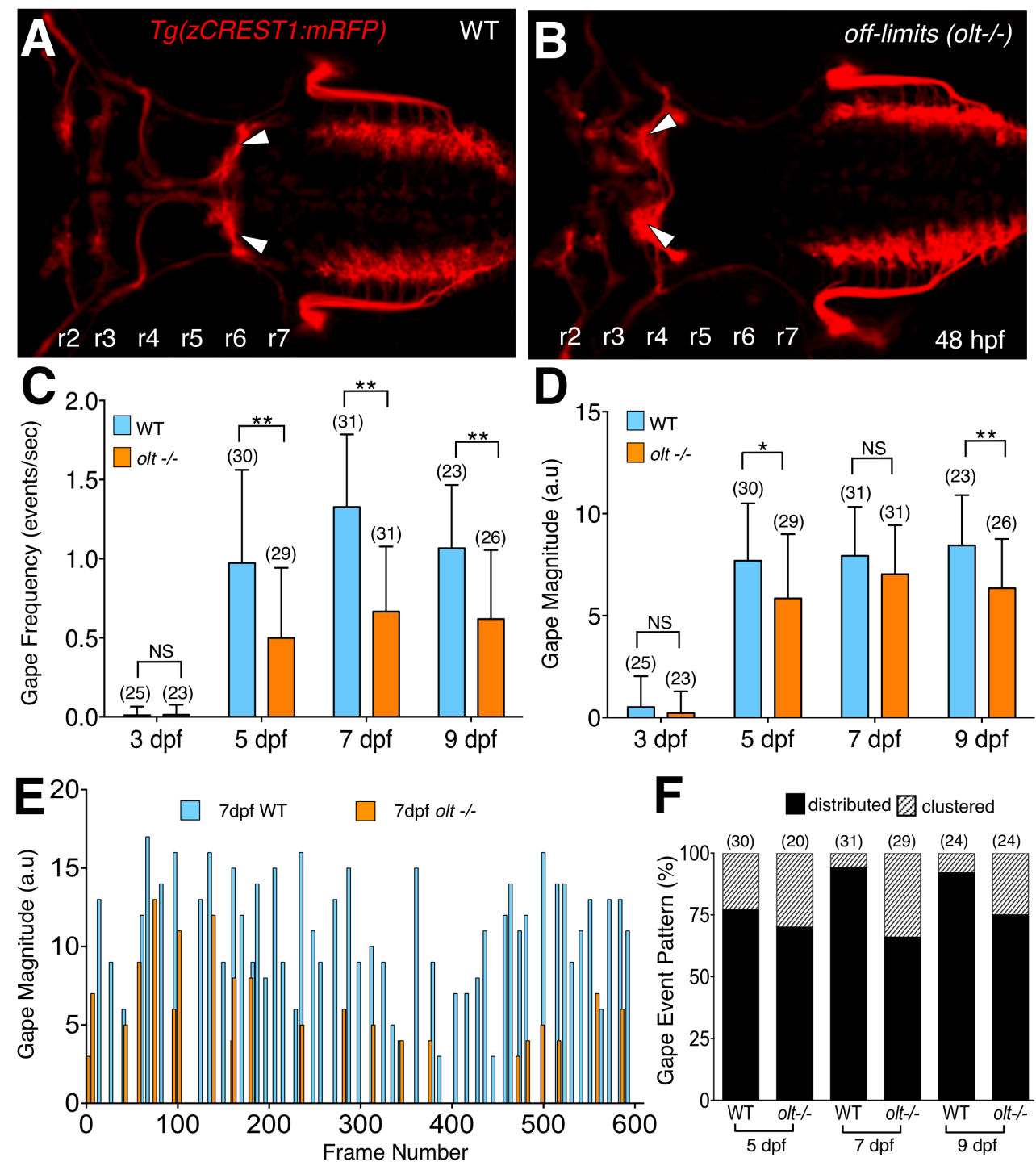
Figure 4.3: olt mutants have reduced jaw movement

(A, B) Dorsal views with anterior to the left of the hindbrain of $48 \mathrm{hpf}$ $T g(z C R E S T 1: m R F P)$ in the olt background. (A) Wildtype (WT) sibling with facial branchiomotor (FBM) neurons (arrowheads) migrating into rhombomere 6 ( $r 6)$. (B) In the olt mutant, FBM neurons (arrowheads) fail to migrate out of r4. (C) Gape frequencies in 5, 7 and $9 \mathrm{dpf}$ olt mutant larvae was significantly reduced compared to wildtype siblings.

Notably,the plateauing of gape frequencies after $7 \mathrm{dpf}$ occurred normally in olt mutants. (D) Gape magnitude was significantly reduced in olt mutants compared to wildtype siblings at 5 and $9 \mathrm{dpf}$, but not at $7 \mathrm{dpf}$. (E) Gape events recorded over a 600 frame period $(\sim 33 \mathrm{sec})$ in representative $7 \mathrm{dpf}$ olt mutants and wildtype sibling larvae showing reduced gape frequency and magnitude in the mutant. (F) Gape event patterns were similarly proportioned between wildtype and olt mutant larvae, with the distributed pattern being the predominant one. Statistical analysis was performed with a two-tailed student t-test $(C, D)$ or Chi-square test $(F)$. NS: not significant, ${ }^{*} p<0.02,{ }^{* *} p<0.001$. Data pooled from 9 experiments (number of larvae in parenthesis). 
Figure 4.4: Defective jaw movements greatly reduce food intake in olt mutants
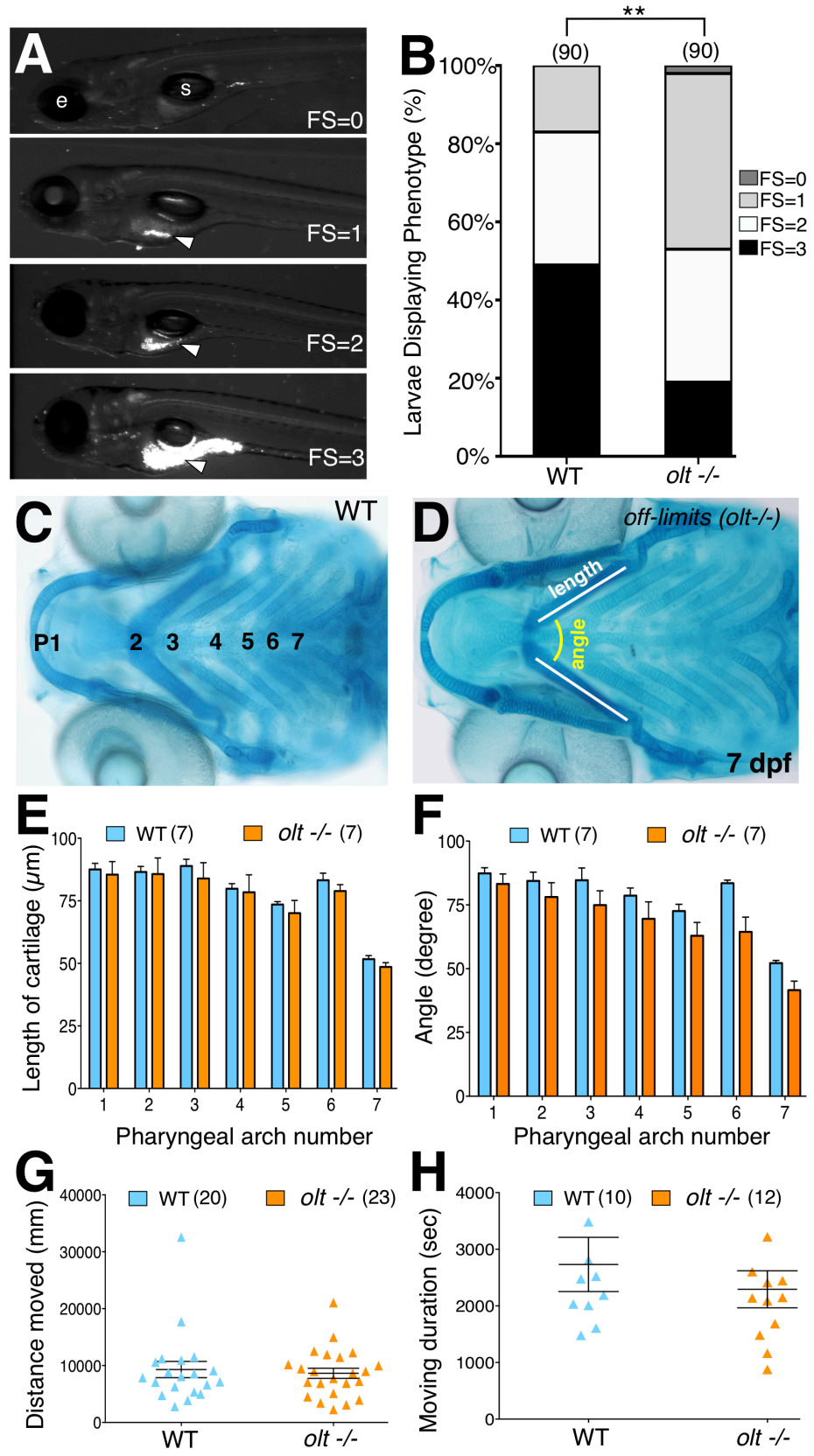
Figure 4.4: Defective jaw movements greatly reduce food intake in olt mutants

(A) A semi-quantitative food intake assay for zebrafish larvae. Lateral views of $7 \mathrm{dpf}$ larvae fed yellow-green fluorescent microspheres coated with fish food for 3 hours. The fluorescent contents (arrowheads) in their guts were visualized under GFP epifluorescence, and observations ranged from no food with a feeding score (FS) of 0 , less than $25 \%$ of the gut $F S=1,50 \%$ of the gut $(F S=2)$, to a full gut $(F S=3)$. e: eye; s: swim bladder. (B) Distribution of feeding scores showing that a population of olt mutants ate significantly less than wildtype siblings. Pooled data from 3 experiments. Chi-square test used for testing significance $\left({ }^{* *} p<0.01\right) .(C$, D) Cartilage morphology at $7 \mathrm{dpf}$ showing that the various elements develop and pattern normally in olt mutants (E, F) Pooled data from 4 experiments. The pharyngeal arch (numbered) was measured for (E) length (white line in $D$ ) and (F) angle (yellow arc in $D$ ) in wildtype siblings and olt mutants. There was no difference in cartilage elements' lengths or the angles subtended by the elements between olt mutants and wildtype siblings. (G, H) Swimming parameters in $6 \mathrm{dpf}$ larvae. (G) Distance moved and $\mathbf{( H )}$ movement duration were analyzed and compared between olt mutant and wildtype sibling larvae with the DanioVision and EthoVision locomotion tracking software (Noldus). There was no significant difference in swimming distance or duration between olt mutants and wildtype siblings. Pooled data from 2 experiments. Number of larvae in 
parenthesis. The two-tailed student t-test was performed to test for significance $(E-H)$. 
Figure 4.5: Axon guidance and outgrowth are unaffected in olt mutant larvae
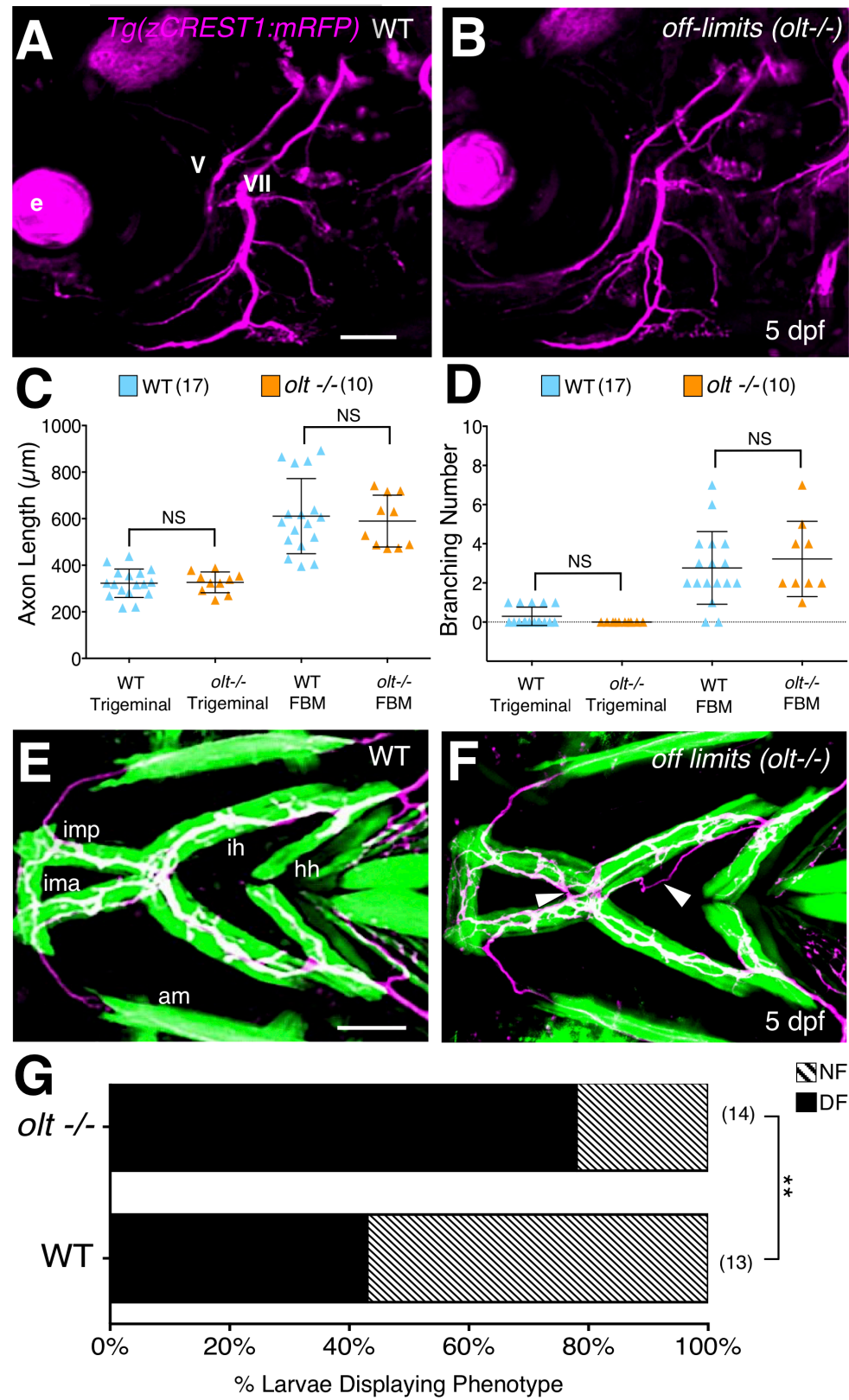
Figure 4.5: Axon guidance and outgrowth are unaffected in olt mutant larvae

(A, B) Lateral view with anterior to the left of the hindbrains of $5 \mathrm{dpf}$ Tg(zCREST1:mRFP) larvae. The morphologies of trigeminal (V) and FBM (VII) axons were analyzed and number of branches were calculated with Leica Application Suite X (LAS X) software. e: eye. (C) The total lengths of trigeminal motor axons and FBM axons and their branches were similar between olt mutants and wildtype siblings. (D) Trigeminal motor and FBM axon branching numbers showed no significant differences between wildtype siblings and olt mutants. Statistical analysis was performed with a two-tailed student t-test (C, D). NS, not significant. Pooled data from 4 experiments (number of larvae in parenthesis). (E, F) Ventral view with anterior to the left of the jaw musculature in $5 \mathrm{dpf}$ $\operatorname{Tg}(z C R E S T 1: m R F P) ; \operatorname{Tg}(a-a c t i n: G F P)$ larvae showing motor axons (magenta) and jaw and gill muscles (green). (E) Thick motor axon fascicles were seen on the ima/imp, ih, and hh muscles in wildtype siblings. This phenotype was defined as Normal Fasciculation (NF). (F) In olt mutants, these axon fascicles appeared thinner and were frequently defasciculated (arrowheads), especially on the in muscle. This phenotype was defined as Defasciculation (DF). (G) There was a preponderance of the defasciculated axon (DF) phenotype in olt mutants, and the proportion of normal fasciculation (NF) to defasciculated (DF) axons was significantly different between wildtype siblings and olt mutants. Statistical analysis 
was performed using Fisher's two-tailed exact test. Data pooled from 4 experiments (number of larvae in parenthesis). ${ }^{* *} p<0.0001$. Abbreviations: ima/imp, intermandibularis anterior/intermandibularis posterior; ih, interhyal; hh, hyohal. Scale bar (A, E), $100 \mu \mathrm{m}$. 
Figure 4.6: Neuromuscular junctions on jaw muscles in olt mutants are unaffected
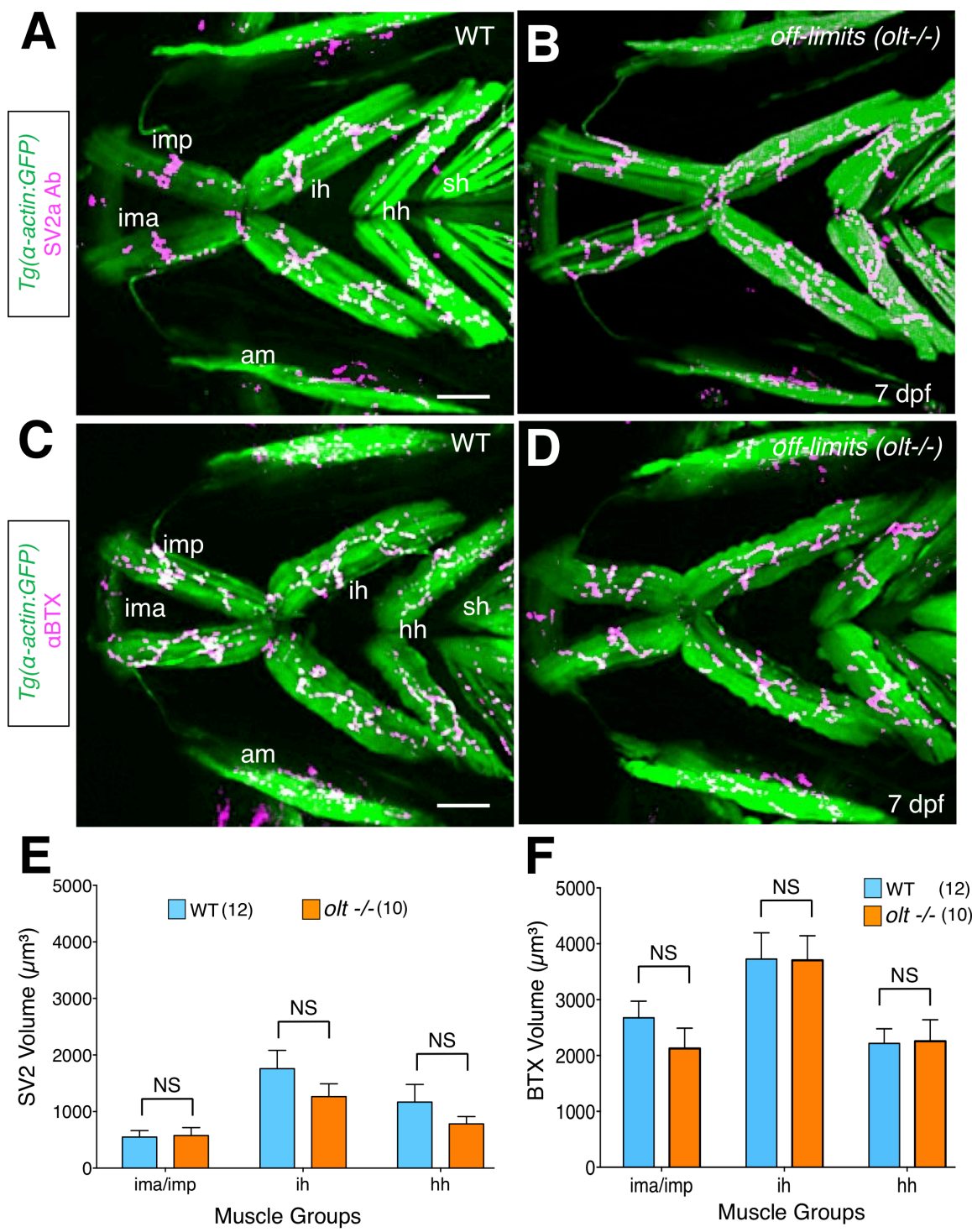
Figure 4.6: Neuromuscular junctions on jaw muscles in olt mutants are unaffected

(A-D) Ventral view with anterior to the left of the jaw musculature in $7 \mathrm{dpf}$ Tg(is/1:GFP); $T g(a-a c t i n: G F P)$ larvae. (A, B) SV2a antibody staining (magenta) of presynaptic structures on the jaw muscles (green). (C, D) Postsynaptic Acetylcholine receptor (AChR) clusters were labeled with alpha-bungarotoxin (aBTX) (magenta) on the jaw muscles (green) in live larvae. Volumes of the presynaptic structures and postsynaptic clusters were calculated from 3D renderings by using the muscle as a mask. There was no significant difference in the volumes of SV2a-stained structures (E) or aBTX-labeled structures $(\mathbf{F})$ on any muscle groups between wildtype siblings and olt mutants. Data pooled from 3 experiments (number of larvae in parenthesis). The two-tailed student t-test was performed to test for significance. NS, not significant. Abbreviations: ima/imp, intermandibularis anterior/intermandibularis posterior; ih, interhyal; hh, hyohal; am, adductor mandibularis. Scale bar (A, C), $100 \mu \mathrm{m}$. 
Figure 4.7: FBM neurons are less active in olt mutants
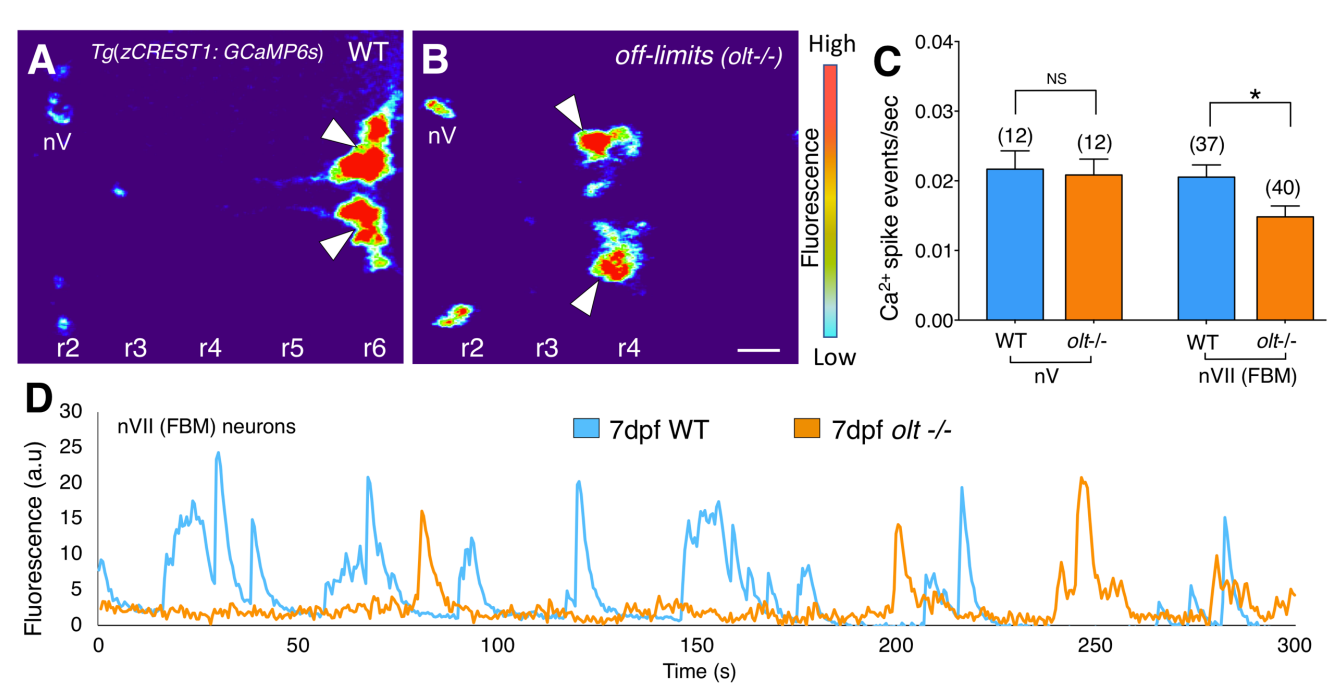
Figure 4.7: FBM neurons are less active in olt mutants

(A, B) Dorsal views, with anterior to the left, of the hindbrains of $7 \mathrm{dpf}$ Tg(zCREST1:GCaMP6s larvae showing fluorescence intensities. (A) In a wildtype larva, fluorescence can be seen in the FBM neurons (arrowheads) in r6, as well as the trigeminal motor neurons (nV) in r2. (B) In an olt mutant, fluorescence is evident in the $\mathrm{nV}$ neurons in $\mathrm{r} 2$ and in the FBM neurons (arrowheads) that have failed to migrate out of $r 4$. (C) The frequency of $\mathrm{Ca}^{2+}$ spike events in FBM neurons was significantly lower in olt mutants compared to wildtype siblings. The $\mathrm{Ca}^{2+}$ spike event frequency for trigeminal motor $(\mathrm{nV})$ neurons was not affected in mutants. Data pooled from 6 experiments (number of larvae in parenthesis). The twotailed student t-test was performed to test for significance, ${ }^{*} p<0.01$. (D) Overlay of GCaMP6s fluorescence traces for a representative wildtype sibling (blue) and an olt mutant (orange) showing fewer Ca2+ spike events in the mutant. Amplitudes of the $\mathrm{Ca}^{2+}$ spikes were not affected. 


\subsection{SUPPLEMENTARY FIGURES AND LEGENDS}

Figure 4.1.1: System for imaging jaw movement and processing steps in motion analysis software

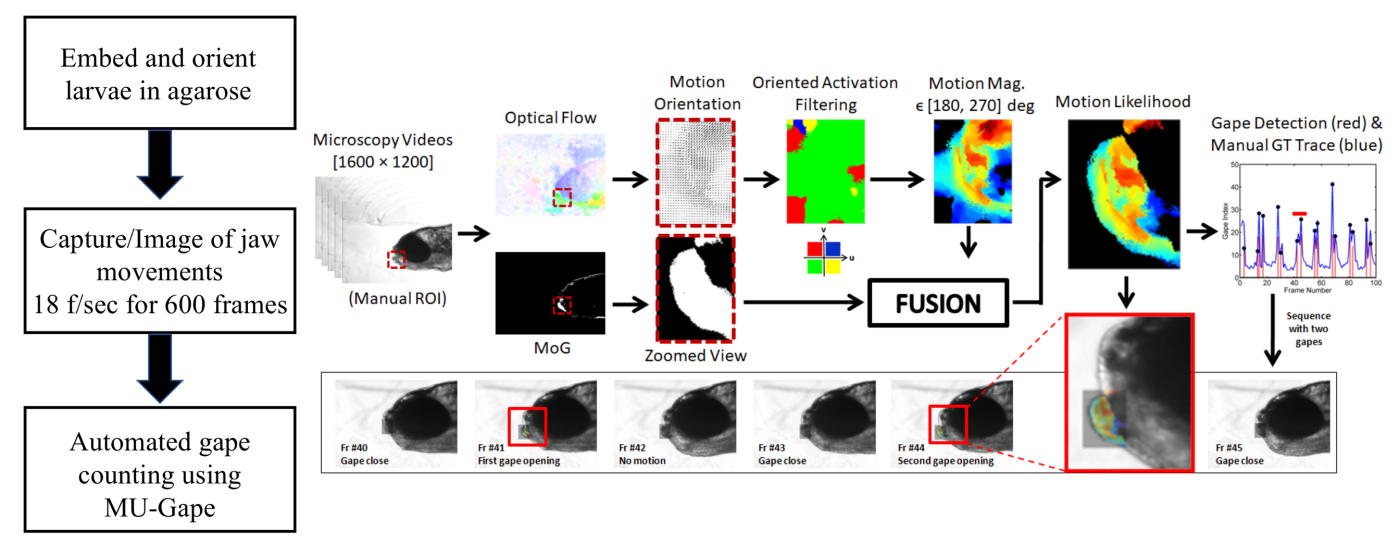


Figure 4.1.1: System for imaging jaw movement and processing steps in motion analysis software

Flowchart of high-throughput video imaging pipeline for larval zebrafish jaw movement and gape frequency analysis using motion estimation and fusion. This computerized motion-based analysis tool produces a digital response for gape or mouth opening of zebrafish microscopy videos in different development stages within a Region of Interest (ROI). ROI is manually specified in the first frame (red box). The system relies on multiple motion-based approaches [optical flow + Background subtraction, (BG)] of jaw movements followed by a robust fusion of motion fields. Optical flow is used to obtain four directional motion maps of the cropped region using the optical flow vectors $(u, v)$, and $B G$ is used to obtain a precise region of motion for the jaw and filter out responses generated by global animal movement within the ROI. The algorithmic fusion fuses BG mask with the motion magnitude map of the flow field within the jaw's desired direction (southwest) and produces the gape response. The software was validated by testing it on 24 videos ( 2400 video frames), with an average accuracy of 98.7 and 1.2 standard deviation, making it robust enough to analyze other videos generated in the study. 
Figure 4.1.2: Morphometric analysis of the heads of 5 and $7 \mathrm{dpf}$ larvae

A
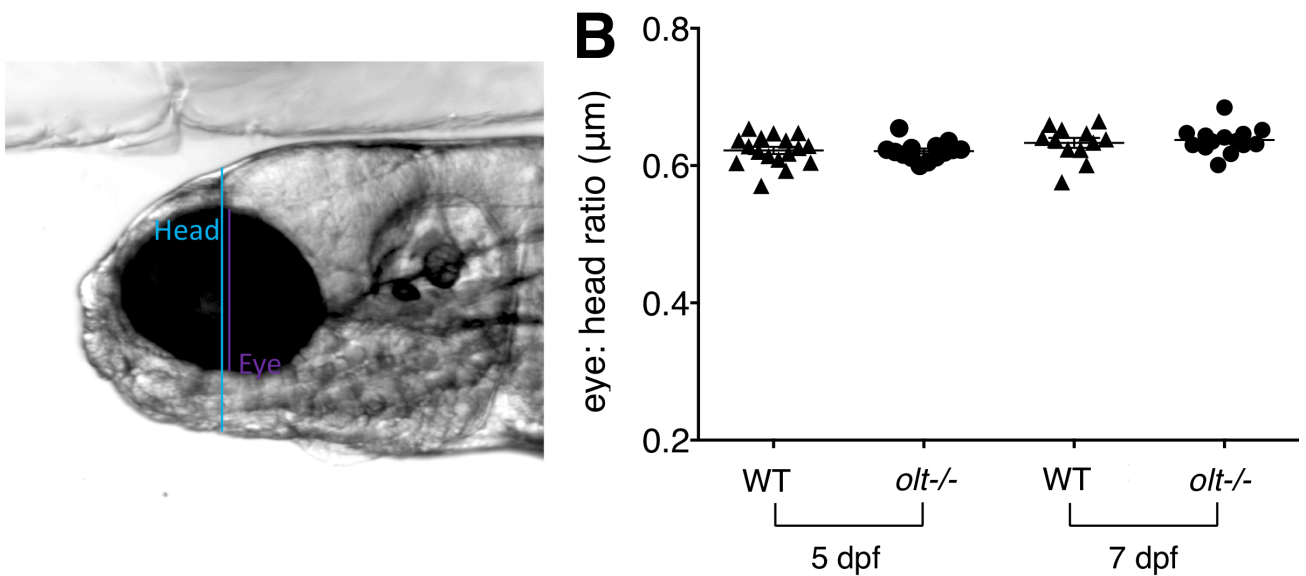
Figure 4.1.2: Morphometric analysis of the heads of 5 and $7 \mathrm{dpf}$ larvae

All measurements were made on single frames captured from time-lapse recordings of laterally mounted larvae. (A) Head height was measured at the level of the epiphysis to the base of the lower jaw. The eye diameter was measured at the same rostrocaudal level as the head height. (B) The ratio of the eye diameter to head height was calculated in 5 and $7 \mathrm{dpf}$ larvae. There were no differences between the two ages for $T g$ (is/1:GFP) larvae, as well as olt mutants and wildtype siblings. Number of larvae is indicated in parenthesis. 
Figure 4.1.3 Measurement of volumes of presynaptic and postsynaptic structures at the NMJs

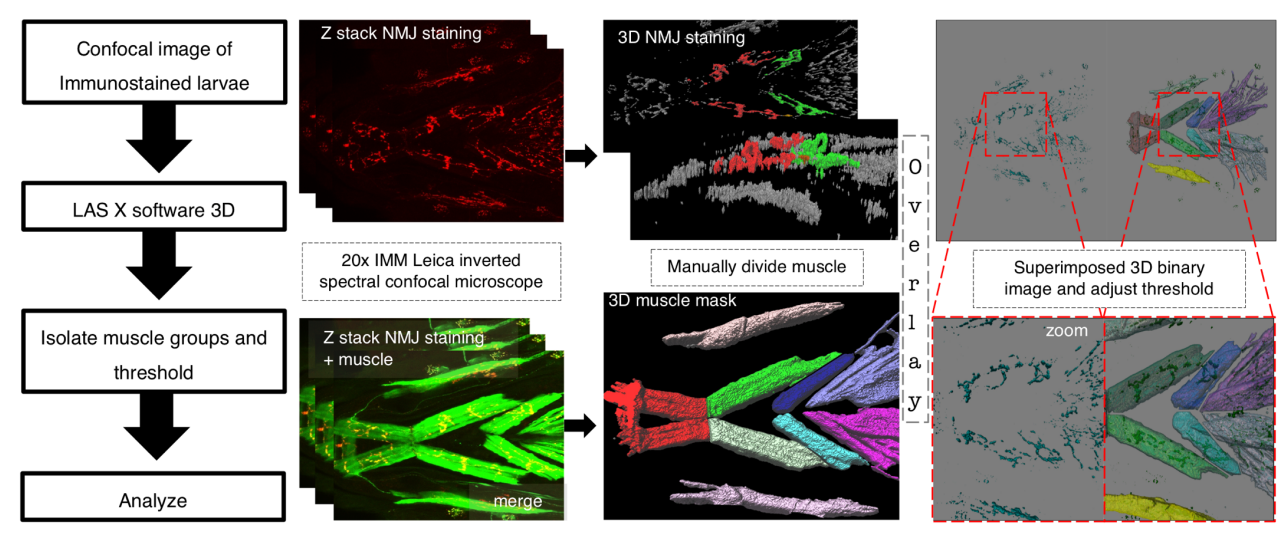


Figure 4.1.3 Measurement of volumes of presynaptic and postsynaptic structures at the NMJs

Flowchart of 3D imaging of $\mathrm{NMJ}$ imaging and analysis. In the image analysis pipeline, Z-stack data files were processed and a new z-stack in 3D was created in LAS X. The newly created 2-channel z-stack stack was opened in the 3D mode in the multi channel. This created two channels "NMJ" and "Muscles" (Left upper and lower panels). Channels were thresholded using the "binary" image (original z-stack image) in a separate window view to adjust the threshold value to the point when all objects in the channels are accurately and precisely defined. The "Draw Straight Lines" method was selected and the lines between the muscle's segments (ima/p, amR, amL, ihR, ihL, hhR, hhL,) were color coded and divided into muscles in the "Binary Image Editing" window only in Muscles channel (Middle lower panel). The "Reference Mask" was marked in the Muscles channel and "Count, Intensity, Area mode" was marked in the NMJ channel (Middle upper panel). The right upper panel shows the binary image of the NMJ channel (left side), and the NMJ channel with overlaid measurement reference mask (right side). The results table with the measurements and summary of all objects within the measured muscles were exported and the new 3D image was saved as a "configuration in 3D" file. 
Figure 4.1.4: Measurement of motor axon lengths and branch numbers
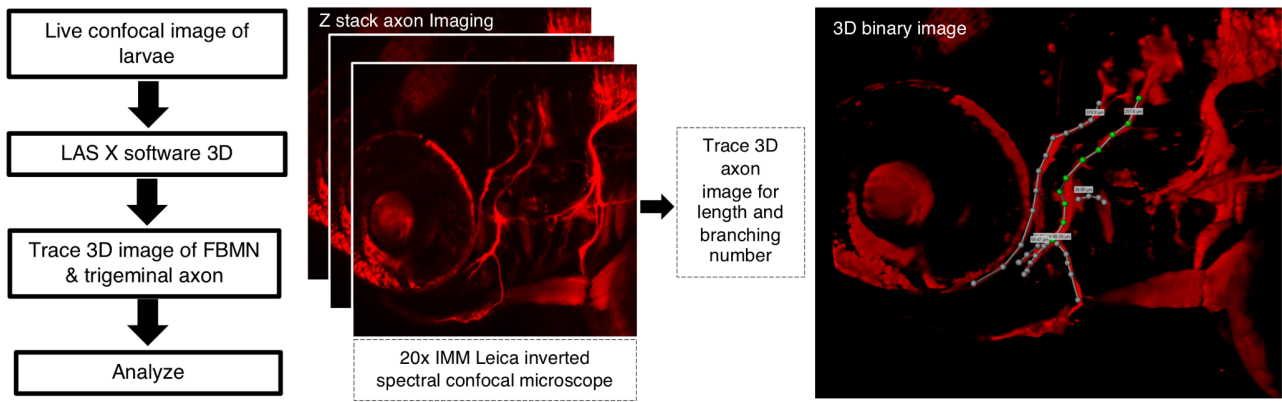
Figure 4.1.4: Measurement of motor axon lengths and branch numbers

Flowchart of axon imaging and analysis using $T g$ (zCREST1:mRFP) larvae. Confocal image stacks were 3D rendered, allowed for manual tracing of trigeminal axon and FBMN axons. The tracing and measurement processes were performed in LAS-X using the "polygon" tool, starting at the hindbrain exit points for both motor axon fascicles. At every step of tracing axons and branches, the 3D images were rotated and viewed at different angles, and the polygon tool was adjusted to ensure that it was on the surface of the axon. Data were exported and traced images were saved as "configuration in 3D" files. 


\subsection{MEDIA LEGENDS}

Media1

Lateral view of a $3 \mathrm{dpf}$ larva, 18 frames per second, 600 frames. Speed up 1.65X.

Media2

Lateral view of a $5 \mathrm{dpf}$ larva, 18 frames per second, 600 frames. Speeded up 1.65X.

\section{Media3}

Lateral view of a $7 \mathrm{dpf}$ larva, 18 frames per second, 600 frames. Real time speed.

\section{Media4}

Lateral view of a $7 \mathrm{dpf}$ wildtype sibling larva, 18 frames per second, 600 frames. Speeded up 1.65X.

Media5

Lateral view of a $7 \mathrm{dpf}$ olt mutant larva, 18 frames per second, 600 frames. Speed up 1.65X.

Media6 
Rotation of image stacks to reveal the 3-dimensional morphologies of the motor axons (top left panel) and their terminations on the jaw muscles (bottom panel). The top right panel shows the polygon tracing method used to measure axon lengths and branch numbers.

\section{Media7}

Dorsal view of the hindbrain of a $7 \mathrm{dpf}$ wildtype sibling. Images of changes in $\mathrm{Ca}^{2+}$ levels in branchiomotor neurons were obtained in the GFP channel at2 frames per second for 5 minutes. Speed up 12.5X.

\section{Media8}

Dorsal view of the hindbrain of a $7 \mathrm{dpf}$ olt mutant. Images of changes in $\mathrm{Ca}^{2+}$ levels in branchiomotor neurons were obtained in the GFP channel at 2 frames per second for 5 minutes. Speed up 12.5X. 


\section{CHAPTER 5: FUTURE DIRECTIONS AND CONCLUSIONS}

The assays for jaw movement (Chapter 3 ) and food intake (Allen et al., 2017) have allowed me to evaluate the functional output of the jaw motor circuit driven by FBM neurons. The zebrafish off-limits (olt) mutant demonstrated that defective FBM neuronal migration does have a profound effect on functional output and subtle effect on circuit organization (Chapter 4). Both jaw movement and food intake are reduced in olt mutants. Furthermore, there are subtle defects in branchiomotor axon fasciculation and FBM neuron activity. These defects could be a consequence of mispositioning of FBM neurons in $\mathrm{r} 4$. However, there are other explanations. First, the olt phenotype may reflect a specific role for the mutated gene $(f z 3 a)$ in the circuit rather than resulting from neuronal mispositioning. I have addressed this possibility through an analysis of another migration mutant landlocked where FBM neurons fail to migrate out of $\mathrm{r} 4$, similarly to olt mutants (5.1). Second, it is possible that neuronal inputs from other brain regions to the branchiomotor circuits are disrupted by mispositioning of the FBM neurons in olt mutants and impacting circuit output. I have performed some studies investigating the role of visual input, and describe ideas for studing inputs from other brain regions for future experiments (5.2). 


\subsection{The Functional Consequences of Defective FBM Neuron Migration In Zebrafish Mutant Landlocked (IIk)}

The landlocked $\left(I / k^{\mathrm{rw} 16}\right)$ zebrafish mutant has a point mutation in the scribble1 (scrb1) gene, which is broadly expressed in the developing nervous system. In $l / k$ mutants, FBM neurons do not migrate from rhombomere (r) 4 to r6. Interestingly, llk homozygous mutant larvae are viable and grow into fertile adults (Wada et al., 2005) suggesting that mutants are able to overcome any functional defects resulting from neuronal mispositioning. Indeed, llk mutant larvae may have normal foraging behavior, and their jaw muscles appear to contract normally (Wada et al., 2005). Another study showed that branchiomotor neurons innervate jaw muscles normally in Ilk mutants, and opercular movement, a behavior driven by an FBM neuron-innervated muscle, was similar to wildtype siblings (McArthur and Fetcho, 2017). Using the jaw movement and food intake assays developed in our lab, I wanted to further investigate whether branchiomotor circuit output was affected in Ilk mutants.

Given that the FBM neurons innervate branchial arch-derived muscles that control jaw and gill movements, a reduction in FBM neuron number and mispositioning of FBM neurons can disrupt food intake. Therefore, we evaluated food intake in llk mutants using the feeding assay (Allen et al., 2017). Food intake in the llk mutant population was significantly reduced compared to wildtype. While $\sim 20 \%$ of $/ l k$ mutant larvae ate just as 
well as most wildtype larvae, the distribution was greatly skewed toward low food intake in mutants compared to wildtype siblings (Fig. 5.1).

Given the poor food intake in Ilk mutant larvae, we wondered whether jaw movement was also reduced. Interestingly, Ilk mutants exhibited significant increase in gape frequency at $5 \mathrm{dpf}$ and $7 \mathrm{dpf}$ compared to their wildtype siblings. At $7 \mathrm{dpf}$, the age in which the feeding experiment is conducted, the gape frequency in mutants remains elevated (Fig. 5.2A). Gape magnitude was not significantly different between mutants and wildtype siblings (Fig. 5.2B). These data suggest that the reduced food intake in Ilk mutants may result hyperactive, rather than reduced, jaw movement. Our $I l k$ phenotypes are preliminary and need further characterization.

If the food intake and jaw movement phenotypes of $I / k$ mutants are confirmed with additional experiments, we will perform studies similar to those carried out with olt mutants (4). We will examine the muscle and cartilage at the lower jaw for defects. We will also examine axon pathfinding to target muscles and the neuromuscular junction for defect in pathfinding and morphology respectively. Food intake defect seen in llk mutants could be due to swimming activity, although all larvae were observed to be swimming normally. Additionally, a previous study stated r6 derived otic and lateral line efferent (OLe) neurons fail to migrate in Ilk mutants (Wada et al., 2005). Thus, the feeding defect seen in feeding could also be due to FBM neuron and OLe neuron aberrant migrations. 
Our observations with olt and Ilk mutants are intriguing. Olt and $I / k$ mutants exhibit a similar failure of migration of FBM neurons, which remain in r4. However, the functional output defects in the branchiomotor circuits are different. Given the importance of neuronal migration-and thus positioning in the developing cortex, the variation in functional output in olt and $I / k$ mutants was striking. The similar decrease in food intake in the mutants was not surprising; therefore, we expected similar defects in the jaw movement assay. The differential effects on gape frequency at 5 and 7 dpf in Ilk compared to olt mutants was striking. McArthur and Fetcho (2017) found the FBM cell bodies and dendrites were mispositioned in the mutants, yet the organization of FBM neurons within the motor nucleus according to target muscle and age was not affected compared to their wildtype siblings. The increase in jaw movement could be due to a level of functional resilience owning to the FBM neuron's position. Importantly, the olt and Ilk data indicate that while FBM neurons are similarly mispositioned in the two mutants, there can be different effects on the functional output of the branchiomotor circuits in the two. It will be of interest to examine branchiomotor circuit output in other zebrafish mutants where FBM neurons exhibit varying levels of defective migration.

\subsection{Neuronal Inputs to Branchiomotor Neurons From Other Brain Regions}


The branchiomotor neurons innervate jaw, face, and neck muscles in humans, and in zebrafish innervate the jaw muscles and gills (Chandrasekhar, 2004; Guthrie, 2007). The zebrafish olt and Ilk mutants demonstrated that defective FBM neuronal migration does impact functional output. The differences in the jaw movement phenotypes of olt and $I / k$ mutants could result from variations in inputs to the branchiomotor neurons from other brain regions. These brain regions include the hypothalamus, and the olfactory, visual, and gustatory systems which all have the ability to regulate food intake (Fig. 5.3 A). In an initial effort to address the roles of these inputs, I have studied the effects of modifying visual system input on the functional output of the branchiomotor circuit.

\subsubsection{Input From The Visual System to the Branchiomotor Circuits}

A previous study has shown that an increase in water turbidity negatively affects the foraging efficiency of zebrafish; the feeding latency increases with the increase in the murkiness of the water (Sekhar et al., 2019). Furthermore, studies on Lake Victoria's cichlid fish species have demonstrated that murky water resulting from human activity can impact mate decision due to color vision loss, which disrupts sexual selection and genetic diversity in the species (Seehausen et al., 1997). Thus, the visual system is heavily relied upon for survival and species diversity.

To address this issue, we have started characterizing jaw movement in bright (Bright) and dark (Dark) conditions. We use bright field (BR) or 
infrared (IR) (dark) illumination (Fig.5.3 B,C) using the nacre zebrafish (White et al., 2008). Due to the loss of mitf gene function, this mutant has no melanocytes, allowing us to perform image analysis without using the chemical phenylthiourea (PTU), a pigmentation blocker, which could have confounding effects on the behavior analysis. Jaw movement was imaged and analyzed in nacre larvae as described earlier (Chapter 2). There was a significant increase in gape frequency from $5 \mathrm{dpf}$ to $7 \mathrm{dpf}$ in BF conditions. While gape frequencies in the IR conditions also increased from $5 \mathrm{dpf}$ to 7 dpf, it was not significant (Fig.5.3B). The sharp increase in gape frequency between 5-7 dpf, and the plateauing of the frequencies at $9 \mathrm{dpf}$ occurred in both conditions. At $7 \mathrm{dpf}$ the $\mathbf{B F}$ condition was higher than in the IR condition, however, it was not significant (Fig.5.3B). The effects on gape magnitude were similar at all age groups and light conditions (Fig.5.3C). While there are no major differences in jaw movement in light and dark conditions, these results are preliminary, and more data must be collected in the two conditions to draw firm conclusions. It will also be instructive to examine the influence of light conditions during the food intake assay; currently larvae are tested solely in the BF illumination.

Finally, the defective and different jaw movements in olt and $l / k$ mutants could result from differences in their visual abilities. The olt/fz $3 a$ and $I / k / s c r b 1$ genes are broadly expressed in the developing brain, affecting visual system function and thus foraging. The optokinetic and optomotor assays can be employed to test whether the two mutants have different 
visual responses. Qualitatively different activity patterns from the visual system to the similarly-mispositioned FBM neurons in olt and Ilk mutants could generate different jaw movement behaviors. Given these possibilities, it will be important to characterize the roles of inputs from other brain regions to obtain a comprehensive understanding of the factors that regulate branchiomotor circuit output.

\subsection{Other Studies}

The use of diverse sensory systems for mediating critical ecological relationships such as determining predator and prey (food) is helpful for making survival decisions in animals. The visual system is heavily relied on for prey capture; the behavioral decisions to avoid or approach when it comes to forging decisions is important to survival (Rice and Westneat, 2005; Filosa et al., 2016). Visual information is conveyed to the hypothalamus, and food intake is regulated by the hypothalamus during foraging. Studies have shown that ablation of areas in the hypothalamus abolishes feeding behavior (Muto et al., 2017) and stimulation of the hypothalamus leads to feeding behavior (Ahima and Antwi, 2008). In addition to vision, the ability to taste, gustatory system and the olfactory system can allow for discriminate chemical stimuli (Kasumyan, 2019). The input from other brain regions may regulate the activity and output of branchiomotor neurons. The jaw movement assays have been conducted during starved zebrafish state. Ongoing studies in our lab are assessing the 
behavioral outputs of the FBM neurons by testing water conditioned with food such as flake food and larval "z" food. Additionally we are testing aversive chemical stimuli such as nicotine. Components of pleasant natural food odor could elicit robust responses in larval zebrafish, while anxiogenic chemical stimuli could subdue behavioral responses. The behaviors to specific odors will allow for a comprehensive characterization of the various environmental and internal conditions that can regulate the functional output of the branchiomotor circuits.

\subsection{CONCLUSIONS}

The work of understanding the functional outputs of the branchiomotor circuit impacted by neuronal mispositioning is never truly complete. As described in the preceding chapters, there are experiments still in progress and future directions that this project can achieve. There can be several insights drawn in this and other chapters.

\subsubsection{Tangential Migration Defect in the Developing Brain}

Neurons have two modes of migrating, radial migration or tangential migration. While most neurons migrate radially, some neurons switch their migration to tangential, allowing neurons to migrate far from their site of origin. This pattern of migration contributes to the formation of the complex nervous system. Our work investigated the link between defective tangential neuronal migration of the FBM neurons and its 
functional outputs. This migration defect led to defective behavior, subtle defects in fasciculation, and neuronal activity.

Other studies have shown the consequences of aberrant tangential migration. In neural cell adhesion molecule (N-CAM) mutant mice, tangential migration of the forebrain is defective. N-CAM mutant mice develop severe reduction of the olfactory bulb (Ono et al., 1994). Furthermore, these mice show a striking loss of fasciculation and a loss of orientation of axons (Chazal et al., 2000). The reduction of the olfactory bulb can impact the mice's sense of smell. While there was no phenotypic change in the olfactory bulb of the olt mutants, the regulatory input from the olfactory system to the branchiomotor circuits can be impacted. The FBM axons in olt mutant zebrafish like the N-CAM mutant mice were defasciculated. These defects demonstrate that tangential migration can impact circuit organizations in similar ways.

In a similar study, the Large $^{\text {myd }}$ mouse hindbrain exhibited a defect in tangential neuronal migration of pontine nuclei neurons in the hindbrain. Homozygous Large ${ }^{m y d}$ mice display muscular dystrophy where they present with abnormal posture and a frog-like position of the hindlimbs and severe curvature of the spine (Qu et al., 2006). These studies, along with the work we have presented, suggest that that tangential migration is necessary to provide complexity to the formation of the neural circuits. 
Given that neuronal migration is evolutionarily conserved, research into potential mechanisms linking aberrant neuronal position and defective circuit function are still ongoing. The precise nature of the migratory movements of neurons should cause defects in circuit and neurological function in defective neuronal migration across species. Due to the tightly regulated process, many species would not make it to the formation of the circuit and thus demonstrate functional output. However, the coordinated nature of neuronal migration could also be beneficial. The conserved nature involved in neuronal positioning can have developmental strategies selected that favor adaptive processes that escape major defective circuit or functional output. This example is demonstrated in our data where there was significant functional output defect, yet the circuit defect was subtle. Neuronal networks may not only rely on positioning to guide their functional development.

\subsubsection{Pursuing the Link Between Neuronal Position and Behavior}

The vertebrate brain consists of networks that are critical for mediating behavior. Behavior is strongly influenced by our drive for survival, such as escaping from threats, foraging, and feeding. An objective goal of system neuroscience is to understand how neuronal positioning drives these fundamental behaviors critical for survival. 
Neuronal positioning translating to a functional output of the neuronal circuit is due, in part, to the characteristic cell types and patterns of connectivity critical for the proper function of the brain. The specification of the appropriate type of neuron(s), the generation of the correct number of neurons, the proper positioning of neurons within the correct brain layer, and the neurons' connection to other cell types allow for complex behaviors to occur.

An example of neurons that link directly to a functional output are found in the hypothalamus. The arcuate nucleus of the hypothalamus is important for appetite regulation and hunger and satiety-promoting neurons (Bouret and Simerly, 2006; Skidmore et al., 2008). Defects in neuronal migration of hypothalamic neurons impact the specific goaldirected behaviors that promote hunger and satiety. Another example can be seen in the spinal cord of mammals. Disruption in the positioning of motor pools-individual motor neurons that innervate muscle, results in abnormal motor neuron connectivity (Sürmeli et al., 2011).

Our work has endeavored to explore the precise link between neuronal position and functional output. The zebrafish's simple yet anatomically homologous brain makes it a powerful model system to begin dissecting the link between defective neuronal positioning and defects in neural circuit-controlling behavior. Our work describes the ontogeny of 
larval zebrafish behavior and the abnormal neuronal migration of the FBM neurons. Our work aims to establish a foundation for neuronal position on circuit function. The data will allow us to dissect the functional output of the branchiomotor circuits. While others have conducted feeding assays, this is the first time a feeding assay was conducted alongside jaw movement assay. These assays allowed us to examine the behavioral output of the branchiomotor circuits. The ontogeny data observed in the larval wildtype is novel to the zebrafish field and demonstrates the developmentally reproducible behavioral output of the branchiomotor circuits.

The olt mutant demonstrated that defective FBM neuron positioning could have adverse effects on circuit organization and function. Prior studies failed to detect functional output differences. Our data suggest that branchiomotor circuits may be sensitive to abnormal neuronal positioning. This data adds to our knowledge about the frizzled gene, specifically how a defect in this gene could cause a functional output defect in zebrafish.

The work above has provided novel insight, and further work is needed to continue to bridge the gap in understanding between neuronal positioning and functional output. 


\subsection{FIGURES AND LEGENDS}

Figure 5.1: Ilk mutants have reduced food intake

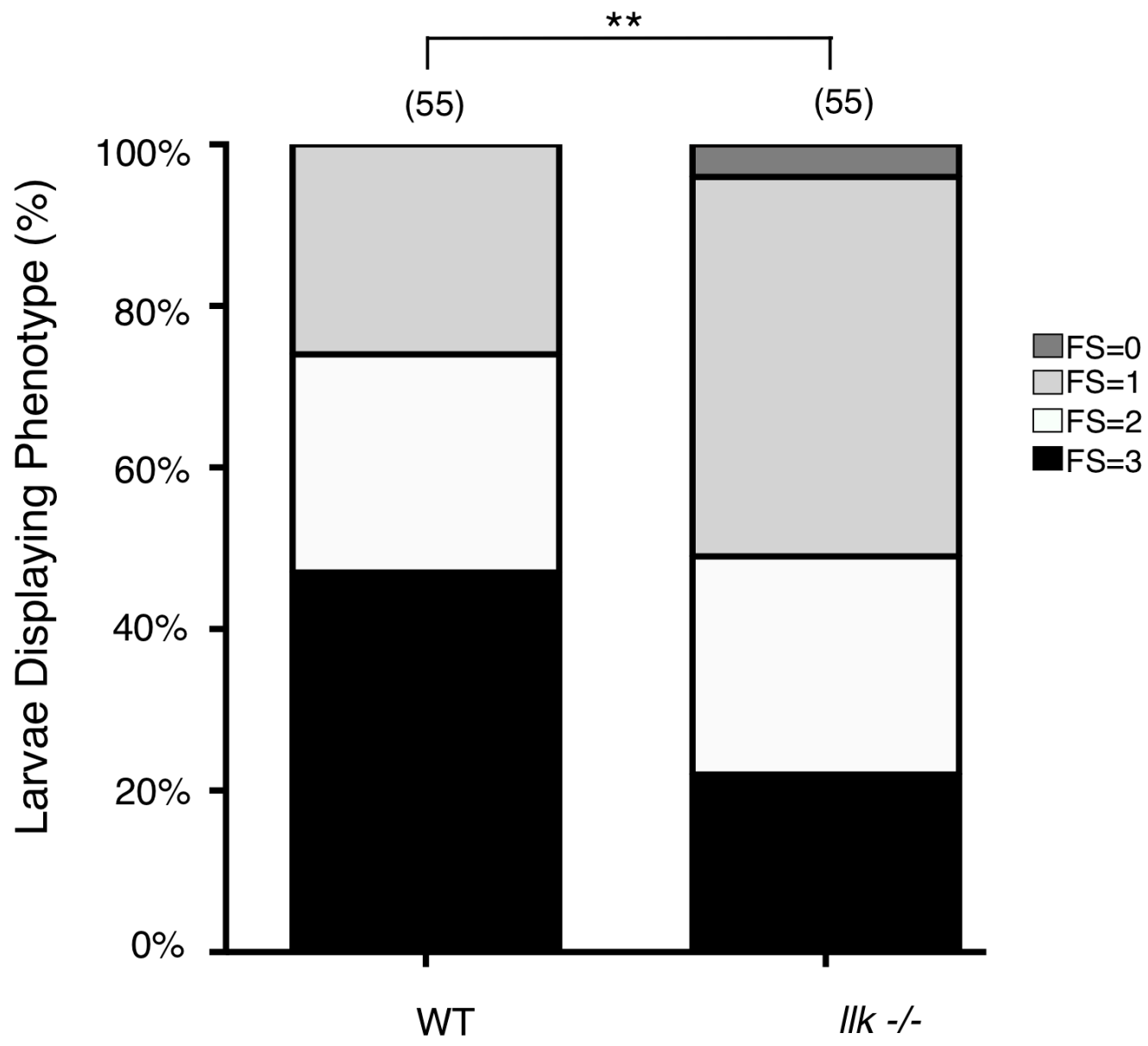


Figure 5.1: Food intake is reduced in landlocked (Ilk) neuronal migration mutant

A quantitative food intake assay for zebrafish larvae. Lateral views of 7 dpf larvae fed fluorescent microspheres coated with fish food for 3 hours. The fluorescent contents in their guts were visualized under GFP epifluorescence and ranged from no food with a feeding score (FS) of 0 , less than $25 \%$ of the gut $F S=1,50 \%$ of the gut $(F S=2)$, to a full gut $(F S=3)$. e: eye; s: swim bladder. (B) Distribution of feeding scores showing that a population of $/ l k$ mutants ate significantly less than wildtype siblings.

Pooled data from 5 experiments. Chi-square test used for testing significance $\left({ }^{* *} p<0.0003\right)$, (number of larvae in parenthesis). 
Figure 5.2: Jaw movement is increased in neuronal migration mutant Ilk
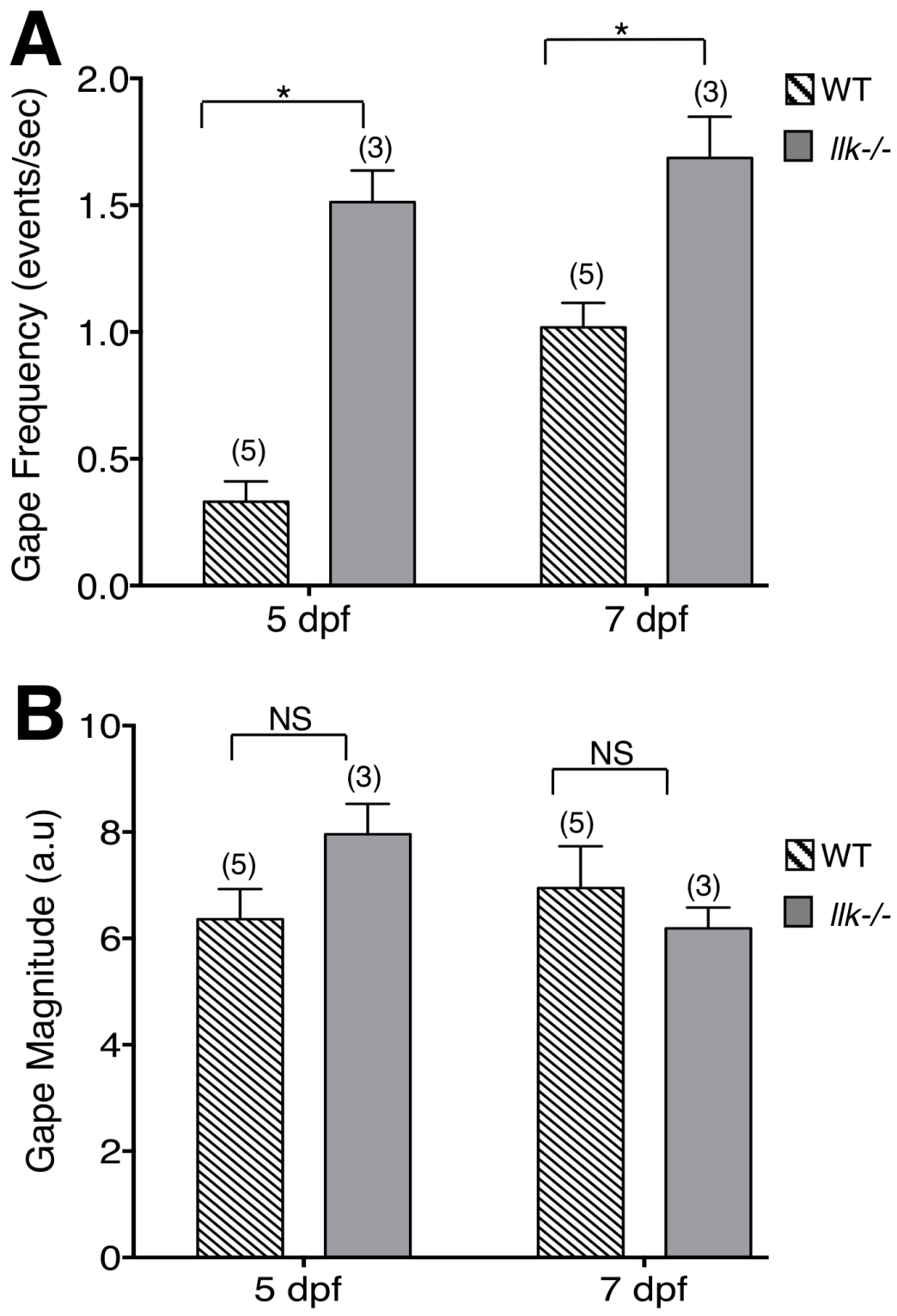
Figure 5. 2: Jaw movement is increased in neuronal migration mutant Ilk

(A) Gape frequencies in 5 and $7 \mathrm{dpf}$ were significantly increased in llk mutant larvae compared to wildtype siblings. (B) Gape magnitude was not impacted between llk mutant larvae and wildtype sibling at any age.

Statistical analysis was performed with a two-tailed student t-test (A, B). NS: not significant, ${ }^{*} p<0.05$. Data from 1 experiment (number of larvae in parenthesis). 
Figure 5.3: Inputs to branchiomotor circuits from other brain regions

A
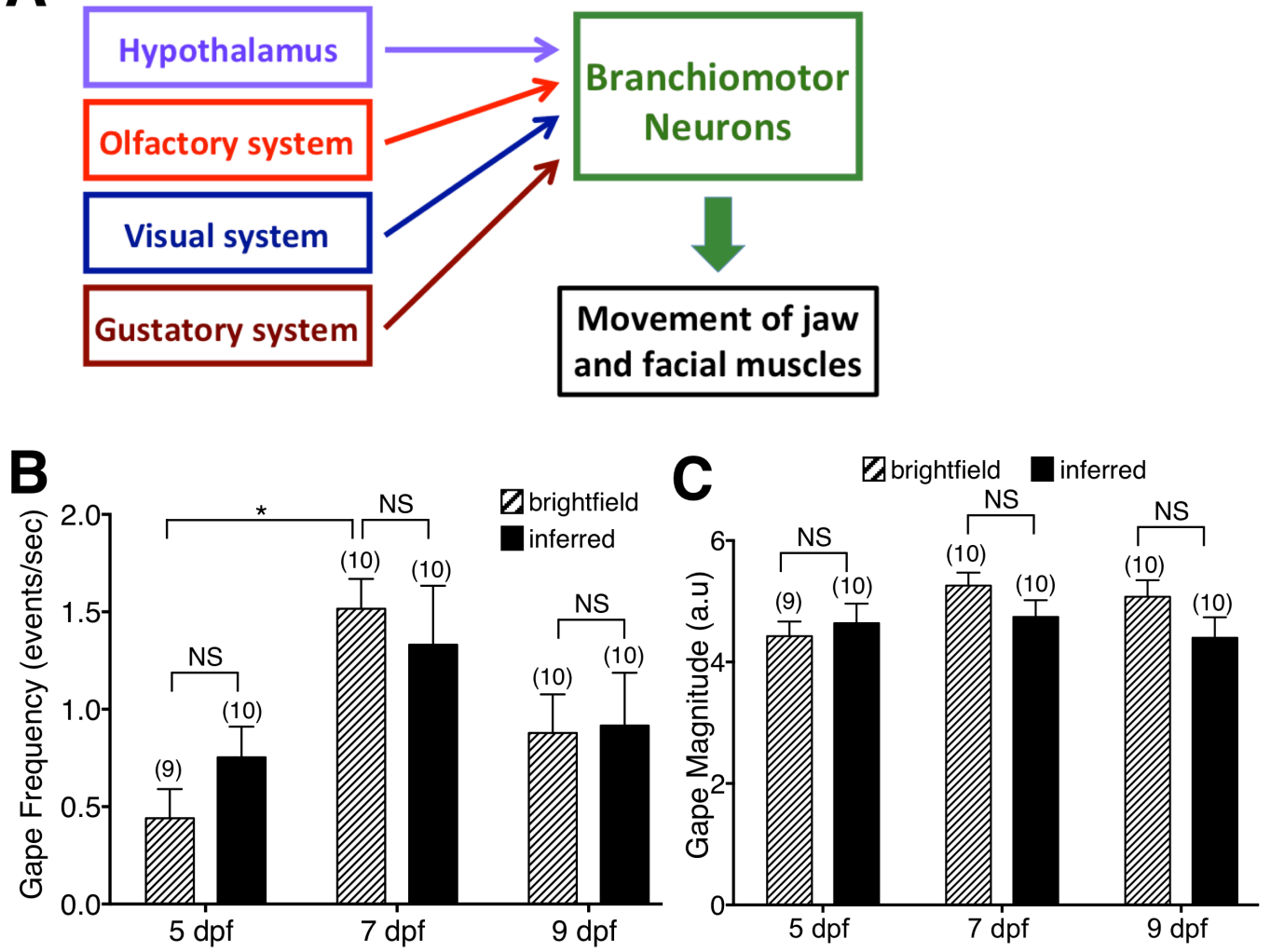
Figure 5.3: Inputs to branchiomotor circuits from other brain regions (A) Flowchart of regulatory system input into the branchiomotor (BM) neurons and the functional output of the BM neurons. (B) Gape frequencies in 5, 7 and $9 \mathrm{dpf}$ larvae in two light conditions brightfield (BF) and inferred (IR). Gape frequencies increase in BF conditions in 5, $7 \mathrm{dpf}$ compared to IR, both light conditions decreased at $9 \mathrm{dpf}$. Statistical analysis was performed with a 2way ANOVA multiple comparison test (A, B). NS: not significant, ${ }^{*} p<0.05$. Data from 1 experiments (number of larvae in parenthesis). 


\section{REFERENCES}

Ahima RS, Antwi DA (2008) Brain Regulation of Appetite and Satiety. Endocrinol Metab Clin North Am 37:811-823.

Allen JR, Bhattacharyya KD, Asante E, Almadi B, Schafer K, Davis J, Cox J, Voigt M, Viator JA, Chandrasekhar A (2017) Role of branchiomotor neurons in controlling food intake of zebrafish larvae. J Neurogenet 31:128-137.

Boulanger-Weill J, Candat V, Jouary A, Romano SA, Pérez-Schuster V, Sumbre G (2017) Functional Interactions between Newborn and Mature Neurons Leading to Integration into Established Neuronal Circuits. Current biology : CB. 27:1707-1720.e1705.

Budick SA, O'Malley DM (2000) Locomotor repertoire of the larval zebrafish: Swimming, turning and prey capture. Journal of Experimental Biology. 203:2565-2579.

Buchsbaum IY, Cappello S (2019) Neuronal migration in the CNS during development and disease: Insights from in vivo and in vitro models. Development 146.

Cario CL, Farrell TC, Milanese C, Burton EA (2011) Automated measurement of zebrafish larval movement. J Physiol 589:37033708.

Carrillo A, McHenry MJ (2016) Zebrafish learn to forage in the dark. Journal of Experimental Biology. 219:582-589.

Chandrasekhar A (2004) Turning heads: development of vertebrate branchiomotor neurons. Dev. Dyn. 229:143-161.

Chandrasekhar A, Moens CB, Warren Jr JT, Kimmel CB, Kuwada JY (1997) Development of branchiomotor neurons in zebrafish. Development. 124:2633-2644.

Copp AJ, Harding BN (1999) Neuronal migration disorders in humans and in mouse models--an overview. Epilepsy Res. 36:133-141.

Dabyns WB, Truwit CL (1995) Lissencephaly and other malformations of cortical development: 1995 update. Neuropediatrics 26:132-147. 
de Rouvroit CL, Goffinet AM (2001) Neuronal migration. Mechanisms of Development. 105:47-56.

Des Portes V, Pinard JM, Billuart P, Vinet MC, Koulakoff A, Carrié A, Gelot A, Dupuis E, Motte J, Berwald-Netter Y, Catala M, Kahn A, Beldjord C, Chelly J (1998) A novel CNS gene required for neuronal migration and involved in X- linked subcortical laminar heterotopia and lissencephaly syndrome. Cell 92:51-61.

Evsyukova I, Plestant C, Anton ES (2013) Integrative Mechanisms of Oriented Neuronal Migration in the Developing Brain. Annu Rev Cell Dev Biol 29:299-353.

Filosa A, Barker AJ, Dal Maschio M, Baier H (2016) Feeding State Modulates Behavioral Choice and Processing of Prey Stimuli in the Zebrafish Tectum. Neuron 90:596-608.

Fleck MW, Hirotsune S, Gambello MJ, Phillips-Tansey E, Suares G, Mervis RF, Wynshaw-Boris A, McBain CJ (2000) Hippocampal abnormalities and enhanced excitability in a murine model of human lissencephaly. J Neurosci 20:2439-2450.

Fox JW, Walsh CA (1999) Periventricular heterotopia and the genetics of neuronal migration in the cerebral cortex. Am J Hum Genet 65:19-24.

Gilmore EG, Herrup K (1997) Cortical development: Layers of complexity. In Current Biology.

Glasco DM, Sittaramane V, Bryant W, Fritzsch B, Sawant A, Paudyal A, Stewart M, Andre P, Cadete Vilhais-Neto G, Yang Y, Song MR, Murdoch JN, Chandrasekhar A (2012) The mouse Wnt/PCP protein Vangl2 is necessary for migration of facial branchiomotor neurons, and functions independently of Dishevelled. Developmental biology. 369:211-222.

Gleeson JG, Walsh CA (2000) Neuronal migration disorders: from genetic diseases to developmental mechanisms. Trends Neurosci. 23:352359.

Gleeson JG (2001) NEURONAL MIGRATION DISORDERS. Ment Retard Dev Disabil Res Rev 171:167-171. 
Gray RS, Roszko I, Solnica-Krezel L (2011) Planar cell polarity: coordinating morphogenetic cell behaviors with embryonic polarity. Developmental cell. 21:120-133.

Graham A, Smith A (2001) Patterning the pharyngeal arches. BioEssays 23:54-61.

Greenwood JSF, Wang Y, Estrada RC, Ackerman L, Ohara PT, Baraban SC (2009) Seizures, enhanced excitation, and increased vesicle number in Lis1 mutant mice. Ann Neurol 66:644-653.

Guarnieri FC, Chevigny A de, Falace A, Cardoso C (2018) Disorders of neurogenesis and cortical development. Dialogues Clin Neurosci 20:255-266.

Guerrini R, Dobyns WB (2014) Malformations of cortical development: Clinical features and genetic causes. Lancet Neurol 13:710-726.

Guthrie S (2007) Patterning and axon guidance of cranial motor neurons. Nat Rev Neurosci 8:859-871.

Hatten ME (1999) Central nervous system. Annu Rev Neurosci 22:539.

Higashijima S, Okamoto H, Ueno N, Hotta Y, Eguchi G (1997) Highfrequency generation of transgenic zebrafish which reliably express GFP in whole muscles or the whole body by using promoters of zebrafish origin. Dev. Biol. 192:289-299.

Higashijima S, Hotta Y, Okamoto H (2000) Visualization of cranial motor neurons in live transgenic zebrafish expressing green fluorescent protein under the control of the islet-1 promoter/enhancer. $J$. Neurosci. 20:206-218.

Hua ZL, Jeon S, Caterina MJ, Nathans J (2014) Frizzled3 is required for the development of multiple axon tracts in the mouse central nervous system. Proceedings of the National Academy of Sciences. 111:E3005-E3014.

Hunter PR, Nikolaou N, Odermatt B, Williams PR, Drescher U, Meyer MP (2011) Localization of Cadm2a and Cadm3 proteins during development of the zebrafish nervous system. Journal of Comparative Neurology. 519:2252-2270.

Jani MP, Gore GB (2016) Swallowing characteristics in Amyotrophic Lateral Sclerosis. NeuroRehabilitation 39:273-276. 
Jiang X, Nardelli J (2016) Cellular and molecular introduction to brain developmen. Neurobiol Dis 92:3-17.

Jonz MG, Nurse CA (2005) Development of oxygen sensing in the gills of zebrafish. J Exp Biol. 208:1537-1549.

Kanatani S, Tabata H, Nakajima K (2005) Neuronal migration in cortical development. J Child Neurol 20:274-279.

Kane JR, Ciucci MR, Jacobs AN, Tews N, Russell JA, Ahrens AM, Ma ST, Britt JM, Cormack LK, Schallert T (2011) Assessing the role of dopamine in limb and cranial-oromotor control in a rat model of Parkinson's disease. J Commun Disord 44:529-537.

Kassim YM, Al-Shakarji NM, Asante E, Chandrasekhar A, Palaniappan K (2018) Dissecting branchiomotor neuron circuits in zebrafish Toward high-throughput automated analysis of jaw movements. IEEE Symp Biomed Imaging:943-947.

Kasumyan AO (2019) The taste system in fishes and the effects of environmental variables. J Fish Biol 95:155-178.

Kato M, Dobyns WB (2003) Lissencephaly and the molecular basis of neuronal migration. Human molecular genetics. 12 Spec No:R8996.

Keays DA, Tian G, Poirier K, Huang G, Siebold C, Cleak J, Oliver PL, Fray M, Harvey RJ, Dear N, Valdar W, Brown SDM, Davies KE, Cowan NJ, Nolan P, Chelly J, Flint J (2007) Mutations in a-Tubulin Cause Abnormal Neuronal Migration in Mice and Lissencephaly in Humans. 128:45-57.

Kimmel CB, Ballard WW, Kimmel SR, Ullmann B, Schilling TF, (1995) Stages of embryonic development of the zebrafish. Dev. Dyn. 203:253-310.

Liu JS (2011) Molecular genetics of neuronal migration disorders. Curr Neurol Neurosci Rep 11:171-178.

Lu J, Sheen V (2005) Periventricular heterotopia. Epilepsy Behav 7:143149.

Lumsden A, Krumlauf R (1996) Patterning the vertebrate neuraxis. Science (80- ) 274:1109-1115. 
Manent JB, LoTurco JJ (2013) Reversing Disorders of Neuronal Migration and Differentiation in Animal Models. Jasper's Basic Mechanisms of the Epilepsies:1129-1138.

Mapp OM, Wanner SJ, Rohrschneider MR, Prince VE (2010) Prickle1b mediates interpretation of migratory cues during zebrafish facial branchiomotor neuron migration. Developmental dynamics : an official publication of the American Association of Anatomists. 239:1596-1608.

Marín O, Rubenstein JLR (2001) A long, remarkable journey: Tangential migration in the telencephalon. Nat Rev Neurosci 2:780-790.

Marín O, Rubenstein JLR (2003) Cell migration in the forebrain. Annu Rev Neurosci 26:441-483.

Marín O, Valiente M, Ge X, Tsai L-H (2010) Guiding Neuronal Cell Migrations. Cold Spring Harb Perspect Biol 2:1-21.

McArthur KL, Fetcho JR (2017) Key Features of Structural and Functional Organization of Zebrafish Facial Motor Neurons Are Resilient to Disruption of Neuronal Migration. Curr Biol 27:1746-1756.e5.

Moffat JJ, Ka M, Jung EM, Kim WY (2015) Genes and brain malformations associated with abnormal neuron positioning. Mol Brain 8:1-12.

Morest DK, Silver J (2003) Precursors of neurons, neuroglia, and ependymal cells in the CNS: What are they? Where are they from? How do they get where they are going? Glia 43:6-18.

Muraki K, Tanigaki K (2015) Neuronal migration abnormalities and its possible implications for schizophrenia. Frontiers in Neuroscience. 9:1-10.

Muto A, Lal P, Ailani D, Abe G, Itoh M, Kawakami K (2017) Activation of the hypothalamic feeding centre upon visual prey detection. Nat Commun 8:15029.

Nadarajah B, Brunstrom JE, Grutzendler J, Wong ROL, Pearlman AL (2001) Two modes of radial migration in early development of the cerebral cortex. Nat Neurosci 4:143-150.

Nadarajah B, Parnavelas JG (2002) Modes of neuronal migration in the developing cerebral cortex. Nat Rev Neurosci 3:423-432. 
Nadarajah B (2003) Radial glia and somal translocation of radial neurons in the developing cerebral cortex. Glia 43:33-36.

Nissen RM, Amsterdam A, Hopkins N (2006) A zebrafish screen for craniofacial mutants identifies wdr68 as a highly conserved gene required for endothelin-1 expression. BMC Developmental Biology. $6: 1-17$.

Noctor SC, Flint AC, Weissman TA, Dammerman RS, Kriegstein AR (2001) Neurons derived from radial glial cells establish radial units in neocortex. Nature 409:714-720.

Noctor SC, Flint AC, Weissman TA, Wong WS, Clinton BK, Kriegstein AR (2002) Dividing precursor cells of the embryonic cortical ventricular zone have morphological and molecular characteristics of radial glia. J Neurosci 22:3161-3173.

Noctor SC, Martinez-Cerdeño V, Ivic L, Kriegstein AR (2004) Cortical neurons arise in symmetric and asymmetric division zones and migrate through specific phases. Nat Neurosci 7:136-144.

Noctor SC, Martínez-Cerdeño V, Kriegstein AR (2008) Distinct behaviors of neural stem and progenitor cells underlie cortical neurogenesis. $J$ Comp Neurol 508:28-44.

O'Rourke NA, Dailey ME, Smith SJ, McConnell SK (1992) Diverse migratory pathways in the developing cerebral cortex. Science (80- ) 258:299-302.

O'Rourke NA, Sullivan DP, Kaznowski CE, Jacobs AA, McConnell SK (1995) Tangential migration of neurons in the developing cerebral cortex. Development 121:2165-2176.

Pan YH, Wu N, Yuan XB (2019) Toward a better understanding of neuronal migration deficits in autism spectrum disorders. Frontiers in Cell and Developmental Biology. 7:1-8.

Pang T, Atefy R, Sheen V (2008) Malformations of cortical development. Neurologist 14:181-191.

Peravali R, Gehrig J, Giselbrecht S, Lütjohann DS, Hadzhiev Y, Müller F, Liebel U (2011) Automated feature detection and imaging for highresolution screening of zebrafish embryos. Biotechniques 50:319324. 
Panzer JA, Gibbs SM, Dosch R, Wagner D, Mullins MC, Granato M, Balice-Gordon RJ (2005) Neuromuscular synaptogenesis in wildtype and mutant zebrafish. In Developmental biology. Vol. 285. 340-357.

Pietri T, Romano SA, Pérez-Schuster V, Boulanger-Weill J, Candat V, and Sumbre G (2017) The Emergence of the Spatial Structure of Tectal Spontaneous Activity Is Independent of Visual Inputs. Cell Rep. 19:939-948.

Portela-Gomes GM, Lukinius A, Grimelius L (2000) Synaptic vesicle protein 2, A new neuroendocrine cell marker. The American journal of pathology. 157:1299-1309.

Rakic $P$ (1972) Mode of cell migration to the superficial layers of fetal monkey neocortex. J Comp Neurol 145:61-83.

Reiner O (2013) LIS1 and DCX: Implications for Brain Development and Human Disease in Relation to Microtubules. Scientifica (Cairo) 2013:1-17.

Reiner O, Carrozzo R, Shen Y, Wehnert M, Faustinella F, Dobyns WB, Caskey CT, Ledbetter DH (1993) Isolation of a Miller-Dicker lissencephaly gene containing $\mathrm{G}$ protein $\beta$-subunit-like repeats. Nature 364:717-721.

Rice AN, Westneat MW (2005) Coordination of feeding, locomotor and visual systems in parrotfishes (Teleostei: Labridae). J Exp Biol 208:3503-3518.

Rinner O, Rick JM, Neuhauss SCF (2005) Contrast sensitivity, spatial and temporal tuning of the larval zebrafish optokinetic response. Investig Ophthalmol Vis Sci 46:137-142.

Seehausen O, Van Alphen JJM, Witte F (1997) Cichlid fish diversity threatened by eutrophication that curbs sexual selection. Science (80) $277: 1808-1811$.

Sekhar MA, Singh R, Bhat A, Jain M (2019) Feeding in murky waters: Acclimatization and landmarks improve foraging efficiency of zebrafish (Danio rerio) in turbid waters. Biol Lett 15.

Slusarski, D.C., and F. Pelegri. 2007. Calcium signaling in vertebrate embryonic patterning and morphogenesis. Developmental biology. 307:1-13. 
Soll DR, Wessels D, Voss E, Johnson O (2001) Computer-assisted systems for the analysis of amoeboid cell motility. Methods Mol Biol 161:45-58.

Song M-R (2007) Moving cell bodies: Understanding the migratory mechanism of facial motor neurons. Arch Pharm Res 30:1273-1282.

Spomer W, Pfriem A, Alshut R, Just S, Pylatiuk C (2012) High-throughput screening of zebrafish embryos using automated heart detection and imaging. J Lab Autom 17:435-442.

Stern O, Marée R, Aceto J, Jeanray N, Muller M, Wehenkel L, Geurts P (2011) Automatic localization of interest points in zebrafish images with tree-based methods. Lect Notes Comput Sci (including Subser Lect Notes Artif Intell Lect Notes Bioinformatics) 7036 LNBI:179-190.

Sun W, Kim H, Moon Y (2010) Control of neuronal migration through rostral migration stream in mice. Anatomy \& Cell Biology. 43:269269.

Sun D, Roth S, Black MJ (2010) Secrets of optical flow estimation and their principles. Proc IEEE Comput Soc Conf Comput Vis Pattern Recognit:2432-2439.

Tau GZ, Peterson BS (2010) Normal development of brain circuits. Neuropsychopharmacology. 35:147-168.

Thoby-Brisson M, Karlen M, Wu N, Charnay P, Champagnat J, Fortin G (2009) Genetic identification of an embryonic parafacial oscillator coupling to the preBotzinger complex. Nature neuroscience. 12:1028-1035.

Thoby-Brisson M, Bouvier J, Glasco DM, Stewart ME, Dean C, Murdoch JN, Champagnat J, Fortin G, Chandrasekhar A (2012) Brainstem respiratory oscillators develop independently of neuronal migration defects in the Wnt/PCP mouse mutant looptail. PloS one. 7:e31140.

Tsai LH, Gleeson JG (2005) Nucleokinesis in neuronal migration. Neuron 46:383-388.

Tsai MH, Muir AM, Wang, WJ. (2020) Pathogenic Variants in CEP85L Cause Sporadic and Familial Posterior Predominant Lissencephaly. Neuron 106:237-245.e8 
van Bruggen HW, van de Engel-Hoek L, Steenks MH, Bronkhorst EM, Creugers NHJ, de Groot IJM, Kalaykova SI (2014) Predictive factors for masticatory performance in Duchenne muscular dystrophy.

Viemari JC, Burnet H, Bévengut M, Hilaire G (2003) Perinatal maturation of the mouse respiratory rhythm-generator: in vivo and in vitro studies. Eur J Neurosci. 17:1233-1244.

Wada H, Iwasaki M, Sato T, Masai I, Nishiwaki Y, Tanaka H, Sato A, Nojima Y, Okamoto H (2005) Dual roles of zygotic and maternal Scribble1 in neural migration and convergent extension movements in zebrafish embryos. Development 132:2273-2285.

Wada H, Tanaka H, Nakayama S, Iwasaki M, Okamoto H (2006) Frizzled3a and Celsr2 function in the neuroepithelium to regulate migration of facial motor neurons in the developing zebrafish hindbrain. Development. 133:4749-4759.

Wang Y, Thekdi N, Smallwood PM, Macke JP, Nathans J (2002) Frizzled3 is required for the development of major fiber tracts in the rostral CNS. The Journal of neuroscience : the official journal of the Society for Neuroscience. 22:8563-8573.

Wang Y (2006) Axonal Growth and Guidance Defects in Frizzled3 KnockOut Mice: A Comparison of Diffusion Tensor Magnetic Resonance Imaging, Neurofilament Staining, and Genetically Directed Cell Labeling. Journal of Neuroscience. 26:355-364.

Westerfield M (1995) The zebrafish book : a guide for the laboratory use of zebrafish (Danio rerio). Eugene, OR: Univ. of Oregon.

White RM, Sessa A, Burke C, Bowman T, LeBlanc J, Ceol C, Bourque C, Dovey M, Goessling W, Burns CE, Zon LI (2008) Transparent Adult Zebrafish as a Tool for In Vivo Transplantation Analysis. Cell Stem Cell 2:183-189.

Yamada M, Hirotsune S, Wynshaw-Boris A (2010) A novel strategy for therapeutic intervention for the genetic disease: Preventing proteolytic cleavage using small chemical compound. Int J Biochem Cell Biol 42:1401-1407.

Yamada M, Yoshida Y, Mori D, Takitoh T, Kengaku M, Umeshima H, Takao K, Miyakawa T, Sato M, Sorimachi H, Wynshaw-Boris A, Hirotsune S (2009) Inhibition of calpain increases LIS1 expression and partially rescues in vivo phenotypes in a mouse model of 
lissencephaly. Nat Med 15:1202-1207. 


\section{Vita}

Emilia Asante was born on September 20,1992, in Kumasi, Ghana, to Florence Asante Adu-Agyei and William Adu-Agyei. Emilia came to the US in 1999. She received a combined high school diploma and an associate degree from Bard High School Early College in New York City. She received her BA in Biology from Earlham College in Richmond, Indiana, in 2014. This work was completed under the guidance of Dr. Anand Chandrasekhar at the University of Missouri, Columbia. Emilia will join the lab of Andy Kodani at St. Jude's Children Hospital in 2021.
\end{abstract}

